\title{
Digital Game as Musical Notation
}

\author{
Paul Turowski \\ Charlottesville, Virginia, USA
}

B.M., Towson University, 2006

M.M., University of Oregon, 2009

\author{
A Dissertation \\ Presented to the Graduate Faculty \\ of the University of Virginia \\ in Candidacy for the Degree of \\ Doctor of Philosophy
}

Department of Music

University of Virginia

May 2016 
(C) Paul Turowski

All Rights Reserved

March 2016 


\begin{abstract}
This dissertation explores the use of game software to structure musical performance with traditional acoustic instruments. The central goal is to promote further study and creative endeavors by 1) suggesting a theoretical framework to inform and contextualize such a practice and by 2) describing a practical methodology. The development of this interdisciplinary field is related to pre-existing tendencies to more fully explore several related continua including composition-improvisation, ludus-paidia, etc.
\end{abstract}

In the first chapter, concepts from which this creative practice has emerged are identified. Chapters 2 through 4 each focus on a particular aspect through a semiotic lens. Throughout, examples by the author as well as by other musicians, artists and game designers demonstrate ways that this practice has manifested and evolved. The final chapter describes the development and realization of a new work, Frontier, that is directly informed by the concepts in this dissertation, and outlines a course for future development. 


\section{Contents}

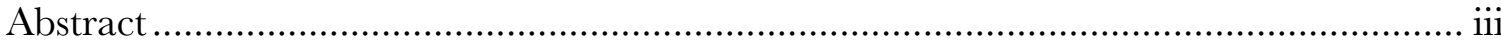

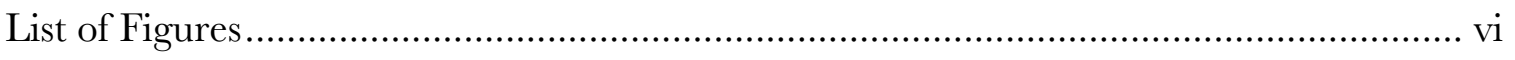

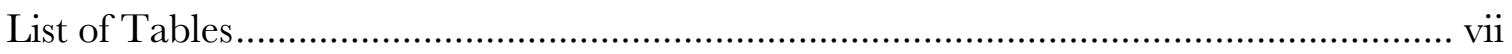

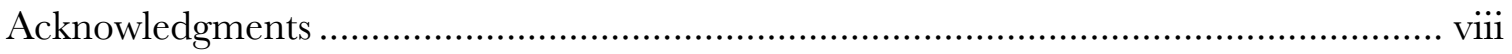

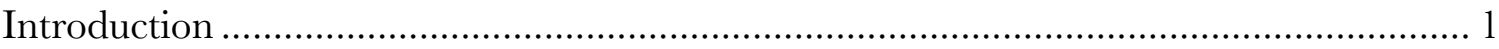

Chapter 1: Context

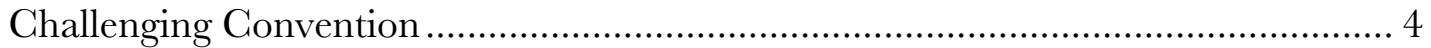

Notation and Composition-Improvisation.......................................................... 8

Multicultural Perspectives........................................................................ 10

Generative Music ........................................................................................... 13

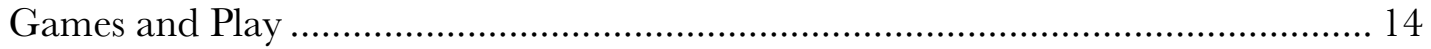

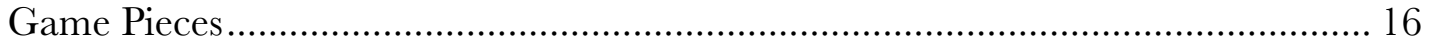

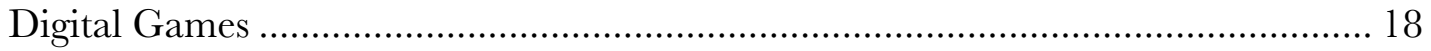

Design Principles for Interactive Systems ....................................................... 19

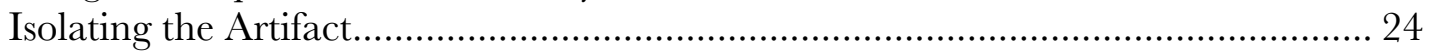

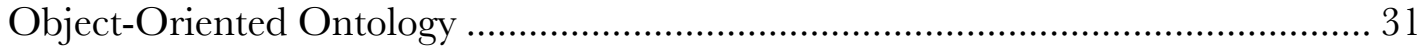

Chapter 2: Player Experience

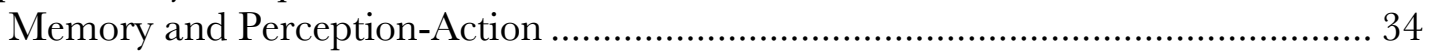

Case Study: Paul Turowski, Study No. 2/2.5 (2011) ............................................... 41

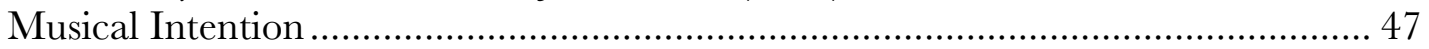

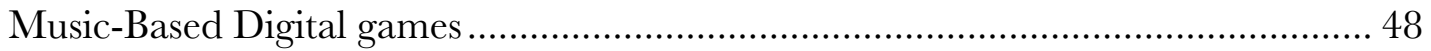

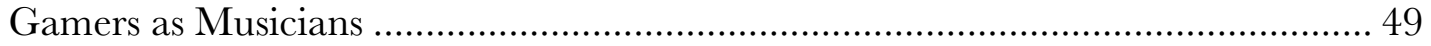

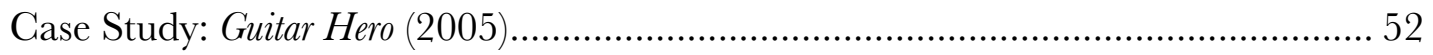

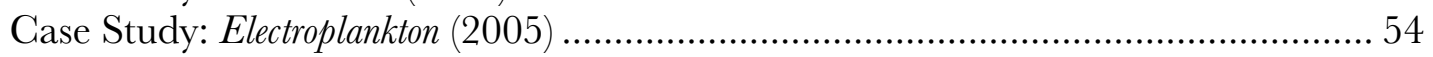

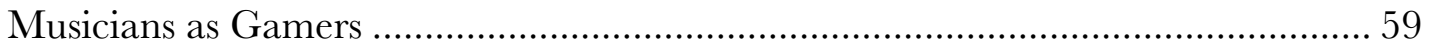

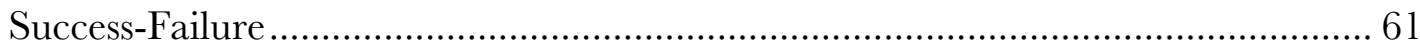

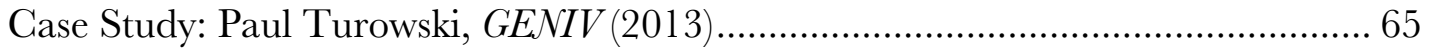

Chapter 3: Dynamic Artifacts

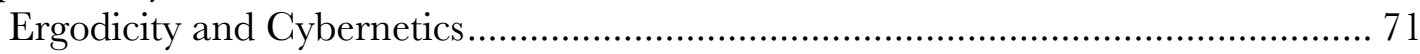

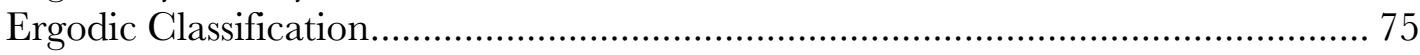

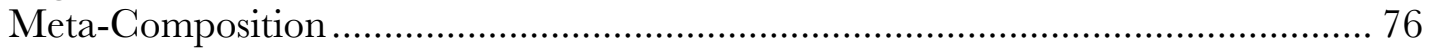

Case Study: Christian Wolff, For 1, 2 or 3 People (1964) ......................................... 78

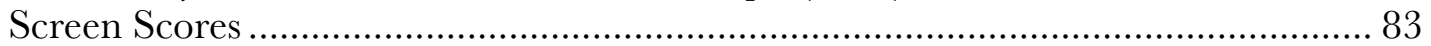

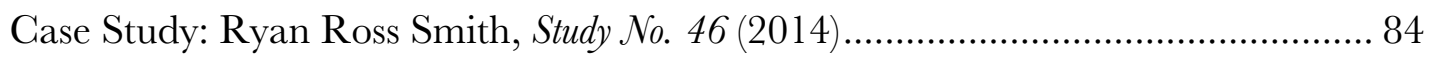

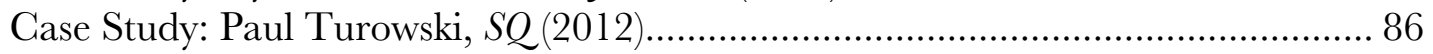

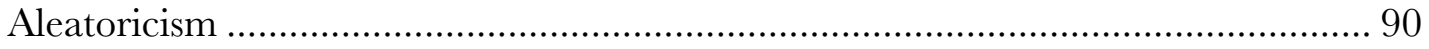

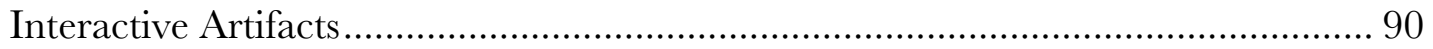

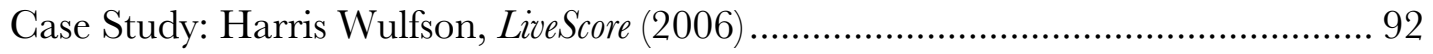


Case Study: Joost van Dongen, Cello Fortress (2012-2016) ..................................... 94

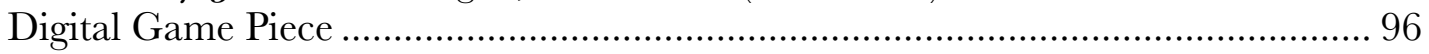

Chapter 4: Poietic Considerations

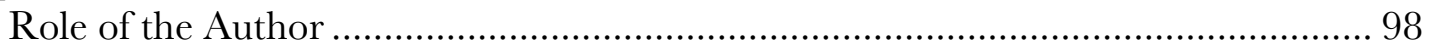

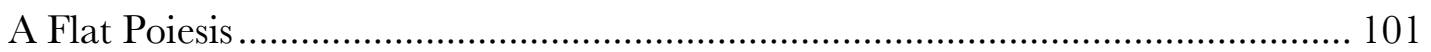

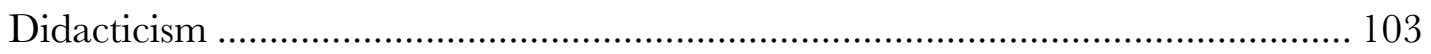

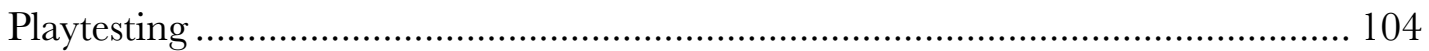

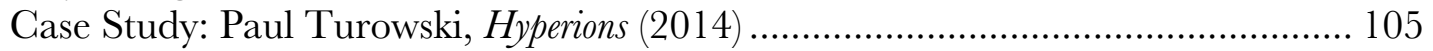

Chapter 5: Frontier and Beyond

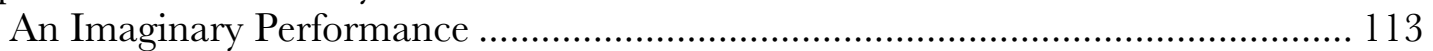

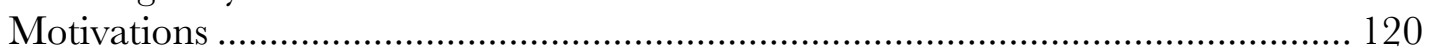

Conceptual Framework for Game Design....................................................... 121

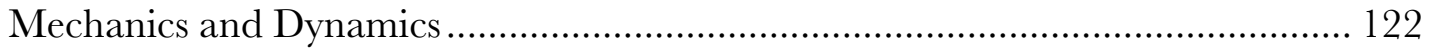

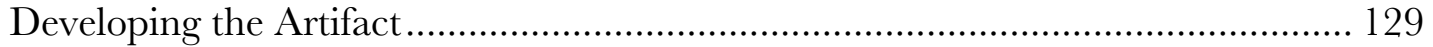

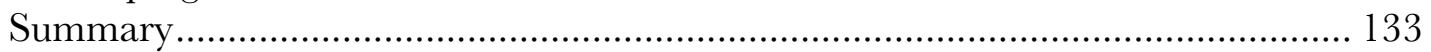

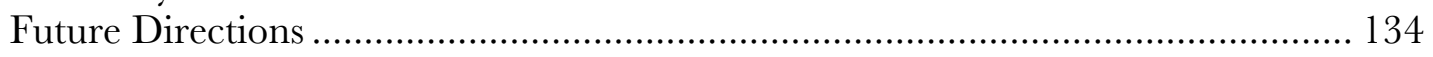

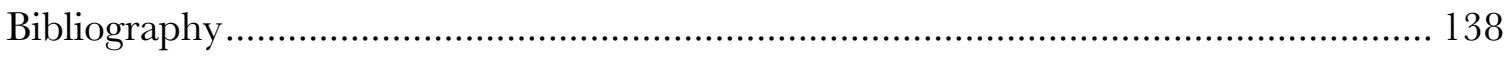




\section{List of Figures}

Figure 1. Salen and Zimmerman's game schema.................................................... 21

Figure 2. Bergun's hierarchy of interactive forms with alteration by Kanaga................. 23

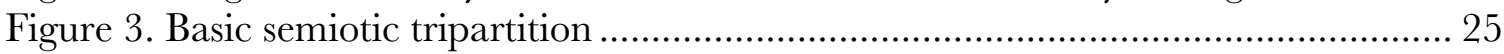

Figure 4. Extended tripartition for musical scores ................................................... 25

Figure 5. Taylor's archive-repertoire concept superposed over the semiotic tripartition . 27

Figure 6. Mechanics-Dynamics-Aesthetics model of game design .............................. 28

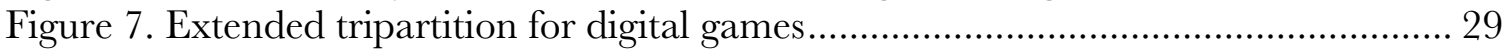

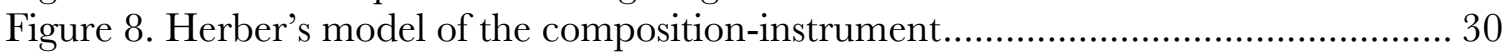

Figure 9. Ermi and Mäyrä's model of immersion....................................................... 31

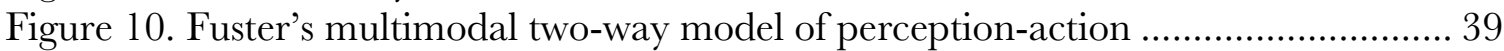

Figure 11. Hutchins's example of a material anchor stabilizing a conceptual blend ....... 40

Figure 12. Turowski, Study No. 2.x. A pitch set is associated with a shape ....................... 41

Figure 13. Turowski, Study No. 2.x. Shapes enter and recede into background ............... 42

Figure 14. Turowski, Study No. 2.x. Waves of color indicate agitation of timbre ............. 43

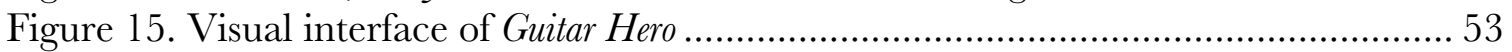

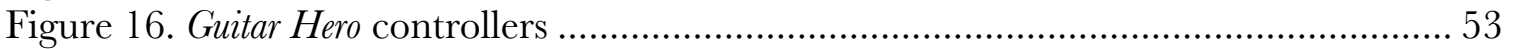

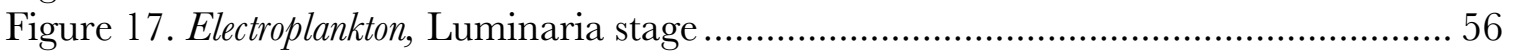

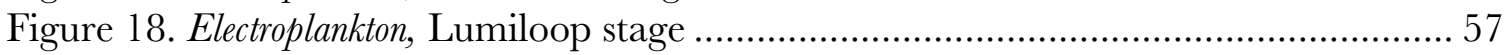

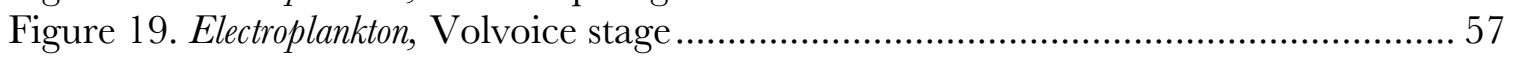

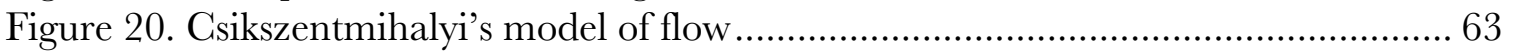

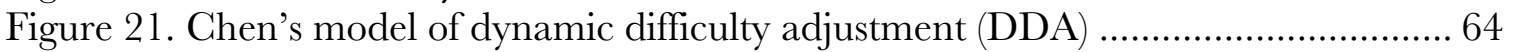

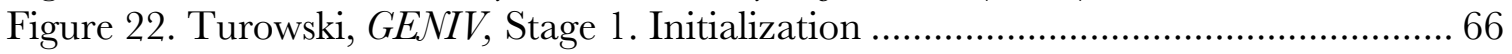

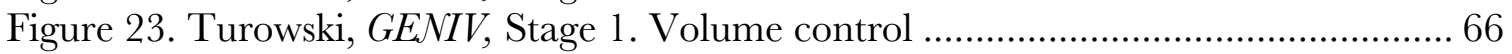

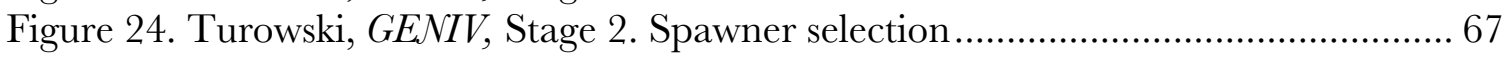

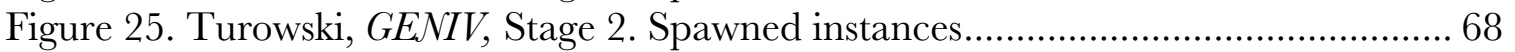

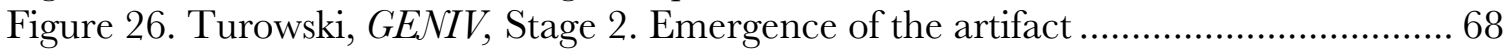

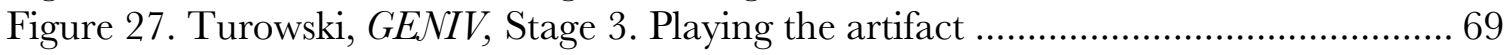

Figure 28. Turowski, GENIV, Stage 4. Improvising with generated clouds .................... 70

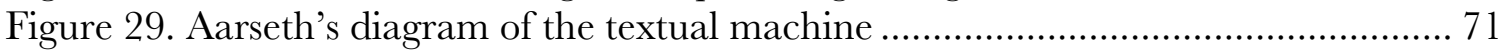

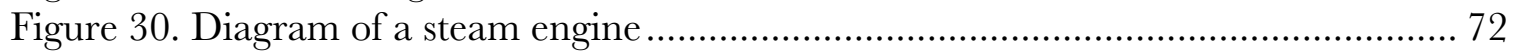

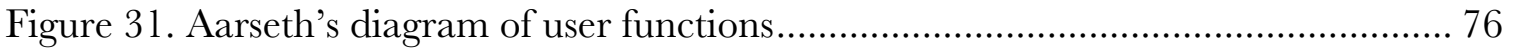

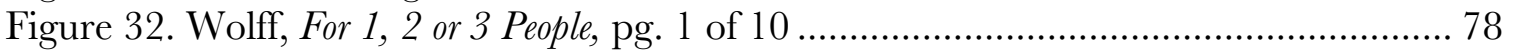

Figure 33. Wolff, For 1, 2 or 3 People. Spatial distribution of events................................ 79

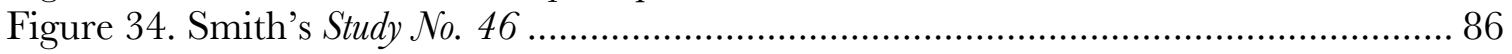

Figure 35. Turowski, SQ. Fixed score component: pitch set for Stage 3 of 5 ................. 87

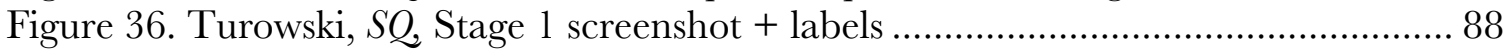

Figure 37. Turowski, $S Q$, Stage 3 screenshot + label............................................... 89

Figure 38. Wulfson, LiveScore. Glient interface .......................................................... 93

Figure 39. Wulfson, LiveScore. Audience interaction knob box ...................................... 93

Figure 40. A performance of van Dongen's Cello Fortress ................................................ 94

Figure 41. Turowski, Hyperions, Creating an ally ..................................................... 106 
Figure 42. Turowski, Hyperions. Allies boosting...................................................... 108

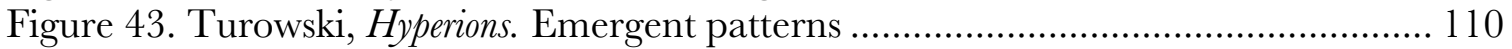

Figure 44. A performance of a revised version of Hyperions at SEAMUS 2015 ............. 112

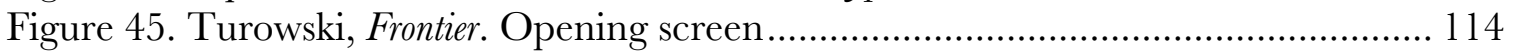

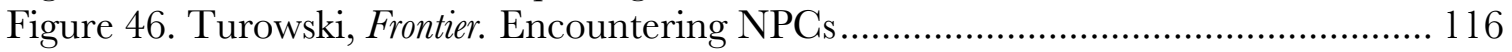

Figure 47. Turowski, Frontier. Breaking hive boundaries ......................................... 117

Figure 48. Turowski, Frontier. Discovering gates and guard NPGs ............................. 118

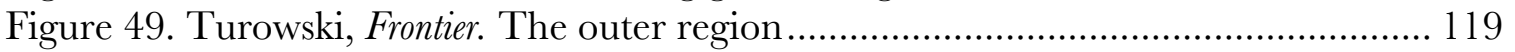

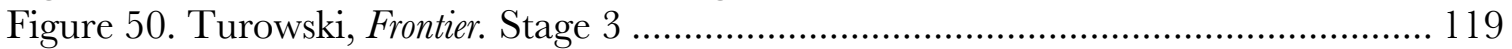

Figure 51. Turowski, Frontier. Three loudness levels ................................................. 124

Figure 52. Turowski, Frontier. Final score as the concluding image ............................. 129

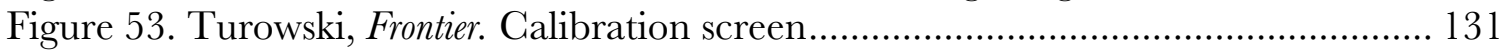

Figure 54. Turowski, Frontier. Title screen ............................................................... 132

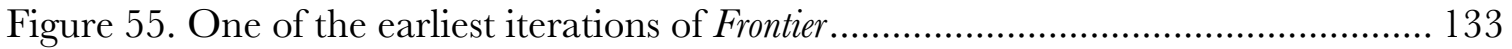

\section{List of Tables}

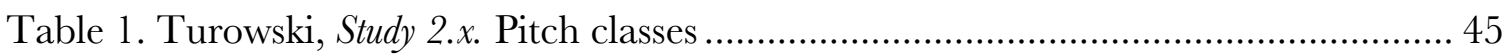

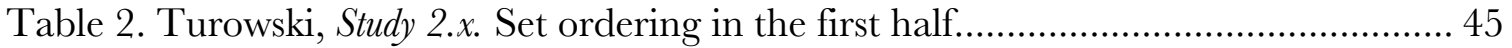

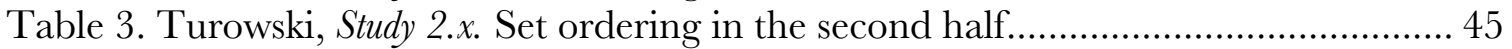

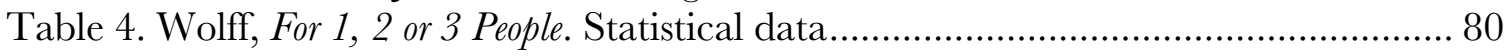




\section{Acknowledgments}

Thanks to my advisor Ted Coffey for guiding the development of this dissertation project since its inception; his wisdom, kindness and sense of humor have inspired and nourished me throughout my time at UVa. I'm grateful to Judith Shatin and Matthew Burtner for serving on my committee and for including me in various creative projects that have allowed me to hone my technical skills. Thanks to Mark Sherriff for serving as the external member of my committee, for welcoming my students and me into his classroom (and game cabinet), and for inviting me to participate in the Game Design Research Group.

Many thanks to Juraj Kojs, whose creative and professional support has been indispensable. Thanks to Peter Bussigel for his keen insights into the realm of intermedia and musical gameplay. I remain indebted to my former composition mentors Jeffrey Stolet and Bill Kleinsasser, whose excellent lessons continue to guide my work to this day.

Thanks to my fellow graduate students - particularly Kevin Davis, Chris Peck, and Kristina Warren - for providing a forum in which to evaluate ideas. Thanks to David Kanaga, Ryan Ross Smith and Joost van Dongen for their valuable correspondences. I'm also grateful to the many video game scholars who gave me helpful feedback at conferences, including Will Gibbons, Neil Lerner, Steven Reale, and Kiri Miller. 
Lastly, thanks to my family - especially my parents, John and Nga, and my brother, Lee- for their continual loving support. 


\section{Introduction}

Over the last ten years or so, gamification - "the use of game design elements in nongame" ${ }^{1}$ or non-play contexts - has become a popular topic of discussion and is prominently described with a utopian/dystopian direness. Jane McGonigal, a staunch advocate of gamification, maintains that "reality is broken" and that games can help us fix it. She imagines a "better and more immersive reality" rather than "better and more immersive alternatives to reality."”2 Alternatively, some critics like Ian Bogost take the stance that "gamification is bullshit," suggesting that "exploitationware" might be a more accurate term to use since in gamification "benefit, honor, and aesthetics are less important than facility."3

If intersections of digital games and musical scores are in some way a form of gamification, must our responses to them be so polarized? Maybe such a question is irrelevant in the case of art music; in art, quality is rarely associated with "facility" or productivity, and games are nothing new. Are our conventional musical practices broken, and if so, are they fixable?

1 Sebastian Deterding, Dan Dixon, Rilla Khaled, and Lennart Nacke, "From Game Design Elements to Gamefulness: Defining 'Gamification'," In Proceedings of the 15th International Academic MindTrek Conference: Envisioning Future Media Environments (MindTrek '11). AGM, New York, NY, USA, 201 1, 9.

2 Jane McGonigal, Reality is Broken: Why Games Make Us Better and How They Can Change the World, digital edition, (New York: Penguin, 2011), Introduction.

3 Ian Bogost, "Gamification is Bullshit," The Atlantic web site, http://www.theatlantic.com/technology/archive/2011/08/gamification-is-bullshit/243338/ [accessed 2016FEB01] 
It seems more likely that the fundamental similarities between musical scores and digital games have naturally set a mutual collision course. Musical scores do not constitute the totality of a musical work; they necessarily require human performance, even if only conceptually. To perform music from a score is to actively take part in processes that reify the musical work. Composers in the postwar era celebrated this notion of the active performer in their "open" musical works. Similarly, digital games are an action-based medium; though they exist objectively (at least partially) on some physical medium like a disc, the intended experience is the active interpretive performance of the player. Like musical (particularly, open) scores, digital games define systems of possibilities to explore and perform.

The potential of digital games to expand existing musical paradigms excites and inspires me. To that end, I look at relevant histories, ontological theories, and various critical assessments to better understand the territory. Some of these concepts are discussed in depth while others are mentioned more briefly as potential paths for further exploration. I also analyze numerous examples - some of which I have created. I include my own works not because I think they are quintessential examples, but because I think they indicate a creative trajectory that might be practically useful for others to consider. All of the ideas presented and works examined - regardless of composer/author, materials, or classification - have significantly shaped the original work that I present in the final chapter. This work, like the ones that precede it, is presented primarily as a point of 
reference. Most of all, I hope that this dissertation inspires further inquiry and experimentation so that we may collectively create work that is more beneficial, honorable, and beautiful. 


\section{Ghapter 1: Context}

\section{Challenging Convention}

At the turn of the $20^{\text {th }}$ century, new philosophical concepts challenged established norms in Western art music. Joel Chadabe describes this period as "the great opening up of sounds" - a shift from the "Absolute Time" of the "Newtonian Period" (1600-1900) to an “asynchronous universe" revealed by science (e.g. Einstein's Special Theory) and embraced throughout the art world. ${ }^{4}$ This is the time when Italian futurists boldly celebrated noise as creative material and Charles Ives experimented with heterogenous "combinational" arrangements. ${ }^{5}$ Artists embraced complexity and multiplicity, and imagined beauty outside of cultural norms to stave off aesthetic atrophy. Ives asks: "If a composer's conception of [their] art, its functions and ideals, even if sincere, coincides to such an extent with these groove-colored permutations of tried-out progressions in expediency so that [they] can arrange them over and over again to [their] delight- - Thave they or have they] not been drugged with an overdose of habit-forming sounds?"6 Luigi Russolo saw the change as a natural progression: "To excite our sensibility, music has developed into a search for a more complex polyphony and a greater variety of

4 Joel Chadabe, Electric Sound: The Past and Promise of Electronic Music, (New Jersey: Prentice Hall, 1997), 21 3.

5 Robert P. Morgan, Twentieth-Century Music: A History of Musical Style in Modern Europe and America, (New York and London: W. W. Norton, 1991), 143.

6 Charles Ives, "Postface to 114 Songs," Essays before a Sonata, and Other Writings, ed. Howard Boatwright (New York: Norton, 1961). 
instrumental tones and coloring. It has tried to obtain the most complex succession of dissonant chords, thus preparing the ground for Musical Noise."7

Similarly, musicians and composers of the postwar era challenged traditional notions of control and performer agency with their open works. According to Umberto Eco, open works require the "auditor ... to do some of this organizing and structuring of the musical discourse. [They collaborate] with the composer in making the composition. ... In other words, the author offers the interpreter, the performer, the addressee a work to be completed."8 The reasons for creating open works are various. For Karlheinz Stockhausen, the inclusion of indeterminate elements in his polyvalent form provided structural variance. Similarly, Pierre Boulez used so-called aleatoric methods to vary the sequence of precomposed sections of music as he favored "highly controlled circumstances."9 Morton Feldman wanted to escape the "obsessive involvement with 'order"' 10 of Stockhausen and Boulez, instead choosing to explore sound more directly, as the abstract expressionist artists around him were doing. Cornelius Cardew's graphic score for Treatise was intended to be performed by "a collection of musical innocents"11 who had various levels of musical experience. Christian Wolff sought to incorporate individual and intersubjective contexts in his open compositions.

7 Luigi Russolo, The Art of Noise (futurist manifesto), trans. Robert Filliou, Great Bear Pamphlet, Something Else Press, 1967. UbuWeb, http://www.ubu.com/historical/gb/russolo_noise.pdf [accessed on 29NOV2014].

8 Umberto Eco, The Open Work, (Cambridge: Harvard UP, 1989), 12, 19.

9 Anne Trenkamp, “The Concept of 'Alea' in Boulez's 'Constellation--Miroir'," Music \&̊ Letters, Vol. 57, No. 1 (Jan 1976), 1.

10 Morton Feldman, "Predeterminate/Indeterminate," in Essays, (Beginner Press, 1985), 49.

11 Cornelius Cardew, "Towards an Ethic of Improvisation" (1968) in Treatise Handbook (London: Peters Edition, 1971). 
In a 1987 article entitled, "Open to Whom and to What," Wolff discusses a common issue with the use of the word 'open' to describe works of art - i.e. the issue of context. The label can have various meanings in just a musical context alone. It could have formal implications such as suggesting "possibilities, multiplicity, heterogeneity, change," or "spontaneity"; it could describe sonic qualities such as a slow tempo, sparseness or a widely-space chord; or it might carry more personal, social and political implications such as being "mediated by nothing other than the naturalized action and reaction of the human body," "open to participation," or somehow shifting the role of audience members from "consumers to participants." He importantly notes that these meanings emerge from a background of context — a background that "can only be constituted by ourselves, our histories which made us, our struggles with those histories, and by these as they are at work at any given time (hearing, performing, scoring, each already active at variously different times) (and place, places)."12

Open works are often lumped together under the umbrella term indeterminate. John Cage identifies the function of the performer of an indeterminate work as "someone filling in color where outlines are given. [They] may do this in an organized way ... [or] in a way which is not consciously organized...."13 Composer Brian Eno suggests a goal-based distinction between open works and indeterminacy:

It is this lack of interest in the precise nature of the piece that has led to the

2 Christian Wolff, "Open to Whom and to What," Interface, vol. 16 (1987), 133-144.

13 John Cage, "Composition as Process: Indeterminacy," Audio Culture: Readings in Modern Music, Eds. Christopher Cox and Daniel Warner, (New York, London: Continuum, 2004), 177. 
(I think) misleading description of this kind of music as indeterminate. I hope to show that an experimental composition aims to set in motion a system or organism that will generate unique (that is, not necessarily repeatable) outputs, but that, at the same time, seeks to limit the range of these outputs. This is a tendency toward a "class of goals" rather than a particular goal, and it is distinct from the "goalless behaviour" (indeterminacy) idea that gained currency in the 1960s. ${ }^{14}$

As aesthetic tastes and compositional methods evolved, so too did the notion of the musical work became an item of scrutiny for scholars. In her historical interrogation of the work-concept and the so-called "imaginary museum of musical works," Lydia Goehr recognizes that the twentieth century "has witnessed increased anxiety over the nature and implications of work-production in the field of music," in part because of its perceived status as an "ontological mutant."15 She claims that Kierkegaard best expresses the reason for such anxiety: "music exists only in the moment of its performance, for if one were ever so skillful in reading notes and had ever so lively an imagination, it cannot be denied that it is only in an unreal sense that music exists when it is read. It really exists only in being produced."16 Philosopher Roman Ingarden claims that the "work is totally different from its score. It is mainly or wholly a sounding work, while the notation of the score is simply a defined arrangement, usually of graphic signs." ${ }^{17}$ Christopher Small advocates a shift from music to musicking - i.e. from noun to verb: "to music is to take

14 Brian Eno, "Generating and Organizing Variety in the Arts," Audio Culture, 227.

15 Lydia Goehr, The Imaginary Museum of Musical Works: An Essay in the Philosophy of Music, (Oxford: Clarendon Press, 1992), 2.

16 Søren Kierkegaard, Either/Or, trans. D. F. Swenson and L. M. Swenson, (Princeton: Princeton UP, 1944), 55.

17 Roman Ingarden, The Work of Music and the Problem of Its Identity, trans. Adam Czerniawski, ed. Jean G. Harrell, (Berkeley, Los Angeles: UC Press. 1986), 2. [orig. Polish 1966]. 
part, in any capacity, in a musical performance, whether by performing, by listening, by rehearsing or practicing, by providing material for performance (what is called composing), or by dancing."18 Nicholas Cook addresses this bifurcation of "music as product" and "music as process," and makes a case for the coexistence of the two as "complementary strands of the twisted braid we call performance."19 He explains the shift toward a "performance studies paradigm" in Western art music, as preceded by the splintering off of theater studies from literary studies, which calls for thinking about musical scores more as "scripts" than "texts." 20 Central to all of these theories is the acknowledgement that a musical work is a complex amalgamation of various forces and materials, of which the score is only one (albeit significant, literally) part.

\section{Notation and Composition-Improvisation}

The evolution of musical notation follows common practice. Kurt Stone identifies three major shifts in the history of Western musical notation: 1) the shift from monody to polyphony around 900 A.D., 2) the shift from partbooks to scores around 1600 to accommodate chords and chordal progressions, and 3) a "stylistic upheaval" in the 1950's that he characterizes by either a desire for more precision or a desire for more ambiguity and indeterminacy. ${ }^{21}$

18 Christopher Small, Musicking (Hanover: Wesleyan UP, 1998), 9.

19 Nicholas Cook, "Between Process and Product: Music and/as Performance," Music Theory Online, Vol. 7, No. 2 (April 2001): 20.

20 Ibid., 15.

21 Kurt Stone, Musical Notation in the Twentieth Century: A Practical Guidebook, (New York, London: W.W. Norton, 1980), xv-xvi. 
Musical composition and free improvisation might be considered opposing terminals on a continuum, upon which all musical performances reside. I broadly define a composition as any pre-arranged plan of action for a performer, whether it specifies a sonic result or performance parameters such as physical position, mental state, etc. (A composition may also exist as a "fixed" medium - e.g. a tape piece-but such a composition is still performed, even if somewhat automatically.) By contrast, the act of improvisation more involves real-time musical decisions by a performer. In the words of Derek Bailey, "improvisation is always changing and adjusting, never fixed, too elusive for analysis and precise description; essentially non-academic. And, more than that, any attempt to describe improvisation must be, in some respects, a misrepresentation, for there is something central to the spirit of voluntary improvisation which is opposed to the aims and contradicts the idea of documentation." 22

This dissertation is less concerned with these terminal points than the continuum that they represent. Composition and improvisation are more directions than they are locations, and any musical event contains both. As David Cope asserts, improvisation "must inherently exist to some extent in all music in which exact notation of every detail is not possible: therefore in all music." 23 According to jazz musician Cecil Taylor:

The eyes are really not to be used to translate symbols that are at best an approximation of sounds. It's a division of energy and another example of

22 Derek Bailey, Improvisation: Its Nature and Practice in Music, (New York: Da Capo, 1992), ix.

23 David Cope, New Directions in Music, (4th ed. Dubuque: Brown, 1984), 242. 
Western craziness. When you ask a [person] to read something, you ask [them] to take part of the energy of making music and put it somewhere else. Notation can be used as a point of reference, but the notation does not indicate the music; it indicates a direction. ${ }^{24}$

On the other hand, even the freest musical improvisation cannot exist without some prearranged plan of action, whether that be instrumentation, venue, or even the decision to not decide about such things.

Any composed piece of music has at least one author. This idea is valuable because context can inform the construction of meaning. However, open scores willfully engage the agency of the performer, complicating the traditional composer-performer relationship and conceding to Cecil Taylor's claim that notation can only ever indicate direction anyway. As Bailey puts it, "the unique experience for a composer in the use of improvisation must be the relinquishing of control over at least some of the music and, even more critically for the composer, passing over that control not to 'chance' but to other musicians. $" 25$

\section{Multicultural Perspectives}

Many conventions in the notation of Western art music are attributed to European traditions, yet the influence of other cultures is undeniable, especially when examining postwar experimentation with openness. For example, Cage's music was heavily

24 Qtd. in Scott DeVeaux and Gary Giddins, Fazz, (New York, London: Norton, 2009), 418.

25 Bailey, Improvisation, 70. 
influenced by his studies of Zen under D.T. Suzuki at Columbia University in 1951. Bailey begins his book on the subject of improvisation by acknowledging the precedence of Indian music, which has directly influenced many prominent Western musicians like George Harrison and John Coltrane.

In a paper titled, "Improvised Music after 1950: Afrological and Eurological Perspectives," George Lewis identifies and examines a distinction between "Afrological" and "Eurological" systems of musical performance in Western culture which is "historically emergent rather than ethnically essential."26 Afrological systems are more associated with improvisation, unique performances and "social instrumentality."27 Improvisation facilitates social instrumentality because it is concerned with "the ability to function in a given context in accordance to each individual's own vibrational flow"- - a concept that Anthony Braxton calls "vibrational affinity dynamics."28 Eurological systems are characterized as being more completely notated - i.e. more toward the composition end of the aforementioned continuum - with the implication that the score is autonomous. ${ }^{29}$ Though open scores seem to blend elements of both cultural systems, Lewis points out that Afrological influence tends to not be acknowledged from the Eurological perspective, as exemplified by Cage's comments about his indifference to

26 George E. Lewis, "Improvised Music after 1950: Afrological and Eurological Perspectives," Black Music Research fournal, Vol. 16, No. 1, (Spring, 1996): 93.

27 Ibid., 94.

28 Anthony Braxton, Tri-axium writings: Vol. 1, (Oakland: Frog Peak Music, 1985), 36.

29 Lewis, "Improvised Music after 1950," 96. 
jazz. ${ }^{30}$ Furthermore, he argues that memory and history, which Cage sought to eliminate in his creative practice, are too important to dismiss in the African-American tradition, which came from a "legacy of slavery and oppression." 31

It's not entirely clear whether Cage's remarks about jazz are a result of ignorance to the cultural implications of his Zen-inspired philosophies or whether there are "exnominative" forces at play. ${ }^{32}$ Practically, both downplay the role of Afrological systems in postwar experiments with composition-improvisation. Of course Cage doesn't speak for all composers of open scores, even within the so-called New York School. For example, Earle Brown began his musical life as a jazz trumpeter and later cited the "breathing space" in jazz as a source of inspiration, even though he confessed boredom with conventional codifications in the jazz community. ${ }^{33}$ Brown's latter comments seem to support the idea that at least some of the indifference toward jazz in the avant-garde Eurological community was directed toward perceived stylistic stasis, as promoted by more conservative musicians like Wynton Marsalis. In any case, the acknowledgement of multiple cultural streams within the already ambiguous and problematic classification of "Western art music" is important. As Gerald Frederic asserts, "one must apprehend the truth concerning the path that real information has traveled and the implications of this

30 Ibid., 99.

31 Ibid., 109.

32 Lewis uses the term "exnomination" - coined by media critic John Fiske - to explain how exclusionary "whiteness" avoids being named to keep from being interrogated and thus changed. Ibid., 100.

33 Bruce Duffie, "Earle Brown Interview with Bruce Duffie," radio interview transcription on web site, http://www.bruceduffie.com/brown.html [accessed on 5DEC2014]. 
in terms of the forces that have been set in motion." ${ }^{34}$

\section{Generative Music}

Inspired by texts on cybernetics, open compositions and scientific inquiry, Eno developed the notion of generative music, which acknowledges that composition can happen from the "bottom-up" instead of "top-down." Generative music is modeled after the evolution of life, the growth of a life form, and the more general observation that "simplicity turns into complexity." This compositional approach considers the composer less as an architect and more as a gardener:

What this means, really, is a rethinking of one's own position as a creator. You stop thinking of yourself as me, the controller, you the audience, and you start thinking of all of us as the audience, all of us as people enjoying the garden together. Gardener included. So there's something in the notes to this thing that says something about the difference between order and disorder ... which I take issue with, actually, because I think it isn't the difference between order and disorder, it's the difference between one understanding of order and how it comes into being, and a newer understanding of how order comes into being. ${ }^{35}$

In generative music, patterns emerge as a result of the rules of the system - a fundamental characteristic that also defines the open score and, to some degree, any compositionimprovisation. Human performers are active agents in such systems, guiding the emergence of sound.

34 Gerald Frederic, "The African Aesthetic in World Creativity: Anthony Braxton's Philosophy of Vibrational Affinity Dynamics," Journal of Black Studies, Vol. 38, No. 2 (November 2007), 133.

35 Brian Eno, "Composers as Gardeners," Edge, http://edge.org/conversation/composers-as-gardeners [accessed on 5SEPT2015]. 


\section{Games and Play}

In Rules of Play, Katie Salen and Eric Zimmerman conduct a thorough comparison of the most cited definitions of game, arriving at their own aggregated version: "A game is a system in which players engage in an artificial conflict, defined by rules, that results in a quantifiable outcome." 36 They define conflict broadly as any struggle that emerges from the circumstances of the game, whether direct or indirect. ${ }^{37}$ According to Jane McGonigal, the most "convincing and useful" definition comes from philosopher Bernard Suits, who wrote, "playing a game is the voluntary attempt to overcome unnecessary obstacles." ${ }^{38}$ She also distills four features common to all games: goals, rules, feedback system, and voluntary participation. Yet another elegant definition can be found in Jesse Schell's Art of Game Design, in which he submits that "a game is a problem-solving activity, approached with a playful attitude." ${ }^{39}$ This is supported by his definition of play as "manipulation that indulges curiosity." 40 Schell also emphasizes the importance of surprise in games, stating that it is "a crucial part of all entertainment - it is at the root of humor, strategy, and problem solving," and that "fun" could be succinctly defined as "pleasure with surprises." ${ }^{\prime 1}$ Surprise runs parallel to the concept of noise in information theory. In the words of Gregory Bateson, "all that is not information, not redundancy,

36 Katie Salen and Eric Zimmerman, Rules of Play: Game Design Fundamentals, (Cambridge: MIT Press, 2004), 80.

37 Ibid., 250.

38 Bernard Suits, Grasshopper: Games, Life, and Utopia, (Boston: David R. Godine, 1990), 41.

39 Jesse Schell, Art of Game Design, (Amsterdam; Boston: Elsevier/Morgan Kaufmann, 2008), 37.

40 Ibid., 30.

41 Ibid., 26-7. 
not form and not restraints - is noise, the only possible source of new patterns." ${ }^{42}$

Roger Caillois identifies a continuum for games that is similar to the aforementioned composition-improvisation continuum. At one extreme is paidia, which describes "spontaneous manifestations of the play instinct" 43 that is "common to diversion, turbulence, free improvisation, and carefree gaiety." 44 On the other side of the continuum is ludus, which manifests "as soon as conventions, techniques, and utensils emerge, [and] the first games as such arise with them." 45 Ludus is honed by "effort, patience, skill, or ingenuity." ${ }^{46}$ Caillois explains that ludus "is complementary to and a refinement of paidia, which it disciplines and enriches." ${ }^{47}$ Furthermore, ludus and paidia "are not categories of play but ways of playing." ${ }^{48}$ To categorize play, he offers four terms: 1) agôn (competition), 2) alea (chance), 3) mimicry (imitation) and 4) ilinx (vertigo).

According to Small, "to play is to change the context of communication, to lift it temporarily from the context of the everyday in order to explore the implications of a relationship or set of relationships without needing to commit oneself to it." ${ }^{49} \mathrm{He}$ also relates musical performance to ritual, adding that, "the more actively we participate, the

42 Gregory Bateson, Steps to an Ecology of Mind, (Chicago, London: Chicago UP, 2000), 416.

43 Roger Caillois, Man, Play and Games, Trans. Meyer Barash, (Urbana, Chicago: Illinois UP, 2001$), 28$.

44 Ibid., 13.

45 Ibid., 29.

46 Ibid., 13.

47 Ibid., 29.

48 Ibid., 53.

49 Small, Musicking, 63. 
more each one of us is empowered to act, to create, to display, then the more satisfying we shall find the performance of ritual." 50 This idea of play as something separate from everyday life is echoed in the notion of the "magic circle," which Salen and Zimmerman borrow from Johan Huizinga's influential book on play, Homo Ludens. ${ }^{51}$ The magic circle is "shorthand for the idea of a special place in time and space created by a game." 52

\section{Game Pieces}

Some composers have created works that feature game-based qualities, such as rules, conflict, and a quantifiable outcome. In his book Formalized Music, Iannis Xenakis describes his foray into heteronomous music, or music with "external conflict." ${ }^{53}$ In his compositions Duel (1958-59) and Stratégie (1962), two conductors each choose one of several musical "events," or sections, in real-time, and the chosen events are played by two separate orchestras. When played simultaneously, the result is that one side wins the conflict, their score (i.e. quantity of points) is incremented, and all proceed to the next round. At the end of an agreed upon number of exchanges or minutes, the results are tallied and one of the conductor/orchestra pairs is declared a winner, receiving a prize. Clearly, Xenakis's conception of game is firmly based in mathematics and game theory.

50 Ibid., 105.

51 Johan Huizinga, Homo Ludens: A study of the Play-Element in Culture, (London: Routledge \& Kegan Paul, 1949).

52 Salen and Zimmerman, Rules of Play, 95.

53 Iannis Xenakis, Formalized Music: Thought and Mathematics in Composition. (Stuyvesant: Pendragon, 1992), 111. 
He defines a "degenerate game" as "one in which the parties play arbitrarily following a more or less improvised route, without any conditioning for conflict, and therefore without any new compositional argument. This is a false game."54 In other words, Xenakis's perspective emphasizes ludus and agôn, while rejecting paidia entirely.

John Zorn had a different perspective on game-based compositions, and wrote a large number of them in the 1970 s and 80 s. His game pieces tied together "loose strings left dangling by composers such as Earle Brown, Cornelius Cardew, John Cage, and Stockhausen" and were "complex systems harnessing improvisers in flexible compositional formats." Zorn also explains that the musicians he was involved with were "excited by the work of Albert Ayler, Anthony Braxton, Leo Smith, and Ornette Coleman," clearly citing Afrological influence in the development of his improvisational language. ${ }^{55}$ These works were created to structure play (paidia)—“to engage, inspire, and enthrall a group of musicians into doing music that they are excited about, so that the excitement is passed on to the audience." Additionally, they do not exist in written form since they were created for specific contexts and since "there is a lot more to these pieces than just the rules." Rather, they are transmitted "as part of an oral/aural tradition" and "there can be no such thing as a definitive edition." 56

Ibid., 113.

55 John Zorn, "The Game Pieces," Audio Culture, 198.

56 Ibid., 196-200. 


\section{Digital Games}

According to Salen and Zimmerman, digital games (a term they use to encompass electronic games, computer games and console-based video games) have four distinguishing traits: 1) immediate but narrow interactivity, 2) manipulation of information, 3) automated control systems, and 4) the potential for networked communication. ${ }^{57}$ Digital games often rely on an electronic visual component, though this is not always the case as demonstrated by audio-only digital games such as Real Sound: Kaze no Regret and the Papa Sangre series. As previously stated, digital games are like musical scores in that they structure player behavior, though they are fundamentally quite different. Unlike traditional musical scores, game react and change based on player actions. As game scholar Espen Aarseth explains:

Games ... are often simulations; they are not static labyrinths like hypertexts or literary fictions. The simulation aspect is crucial: it is radically different alternative to narratives as a cognitive and communicative structure. Simulations are bottom up; they are complex systems based on logical rules.

Games are both object and process; they can't be read as texts or listened to as music, they must be played. Playing is integral, not coincidental like the appreciative reader or listener. The creative involvement is a necessary ingredient in the uses of games. The complex nature of simulations is such that a result can't be predicted beforehand; it can vary greatly depending on the player's luck, skill and creativity. ${ }^{58}$

Digital games are also often described as being "interactive," which some scholars find

Salen and Zimmerman, Rules of Play, 85-91.

58 Espen Aarseth, "Computer Game Studies, Year One," The International Fournal of Computer Game Research, Vol. 1, Issue 1, (July 2001). 
problematic. For example, in Gaming: Essays on Algorithmic Culture, Alexander Galloway explains:

One should resist equating gamic action with a theory of "interactivity" or the "active audience" theory of media. Active audience theory claims that audiences always bring their own interpretations and receptions of the work. Instead I embrace the claim, rooted in cybernetics and information technology, that an active medium is one whose very materiality moves and restructures itself - pixels turning on and off, bits shifting in hardware registers, disks spinning up and spinning down. Because of this potential confusion, I avoid the word "interactive" and prefer instead to call the video game, like the computer, an action-based medium. ${ }^{59}$

Furthermore, "interaction" can imply mutual agency between conscious beings - a quality that our current digital media may simulate but arguably do not (yet) actually achieve.

Nevertheless, the complex causal networks that are inherent in digital games are important to recognize and will be described as interactive in the current context.

\section{Design Principles for Interactive Systems}

In his book The Design of Everyday Things, Don Norman explains that designing interactive systems involves the careful consideration of certain principles. Affordance refers to the relationship between the properties of an object and the capabilities of an agent. While affordances make certain actions possible, constraints make other actions impossible. Norman also asserts that it is the job of the designer to make affordances known with a property he calls a signifier. In his words, "signifier refers to any mark or sound, any

59 Alexander Galloway, Gaming: Essays on Algorithmic Culture (Minnesota UP, 2006), 3. 
perceivable indicator that communicates appropriate behavior to a person." ${ }^{\prime 60}$ Unlike affordances, signifiers are not directly related to the physical properties of an object and can therefore be misleading. Additionally, a well-designed system employs natural mappings and immediate predictable feedback. In digital games, feedback is achieved by mapping the audiovisual stimuli produced by the game to player action. In her book Playing With Sound, Karen Collins coins the term "kinesonic synchresis" (derived from Michel Chion's "synchresis" - a portmanteau of synchronism and synthesis ${ }^{61}$ ) to describe the phenomenon of perceiving sound (or, presumably, any sensory perception) as fused to action. ${ }^{62}$ In accordance with Norman, she asserts that “...repeatability of events is one of the key elements in sound's ability to provide feedback to the player."63 These design principles help establish a conceptual model for the user, which enhances discoverability and understanding.

In game scholarship, rules are often conflated with affordances and constraints, which is potentially problematic. Building on the idea of the magic circle, Salen and Zimmerman describe games using three embedded conceptual schemata: rules, play, and culture.

(Figure 1) Rules concern the formal arrangement of structure, play concerns the experience of the player and their interaction with the game and other players, and culture concerns the cultural context that any game is a part of. They characterize rules by the

60 Don Norman, The Design of Everyday Things, Revised and Expanded Edition, (Perseus, 2013), 14.

61 Michel Chion, Audio-Vision: Sound on Screen, Ed. and trans. Claudio Gorbman, (New York: Columbia UP, 1990), 63.

62 Karen Collins, Playing with Sound, (Cambridge: MIT Press, 2013), 32.

63 Ibid., 33. 
following criteria: rules 1) limit player action, 2) are explicit and unambiguous, 3) are shared by all players, 4) are fixed, 5) are binding and 6) are repeatable. ${ }^{64}$ In a paper on Guitar Hero and Rock Band, Roger Moseley claims that, “...digital games offer rules - which is to say possibilities bound by constraints - for entering into relationships with the world that are simultaneously material and imaginary, real and virtual." ${ }^{\prime 65}$ Similarly, conflict seems to be conflated with feedback at times. Chris Crawford writes, "Conflict can only be avoided by eliminating the active response to the player's actions. Without active response, there can be no interaction. Thus, expunging conflict from a game inevitably destroys the game." 66

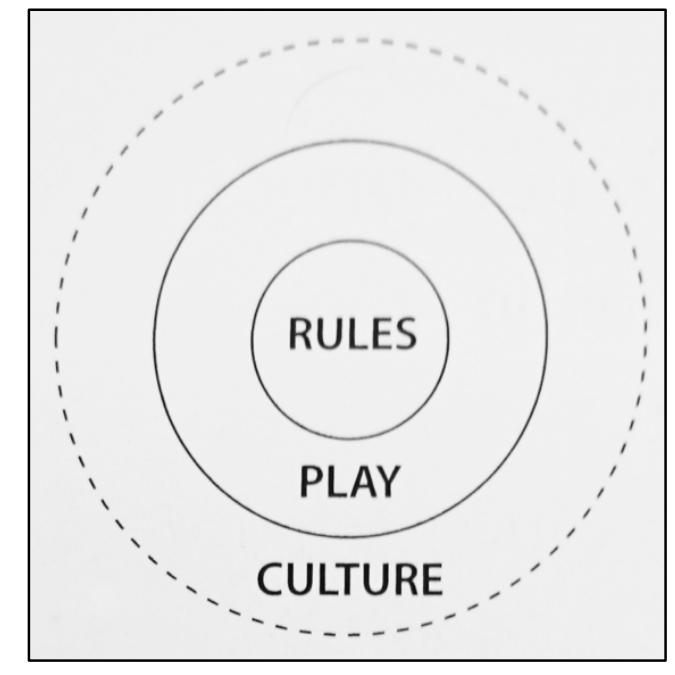

Figure 1. Salen and Zimmerman's game schema ${ }^{67}$

Experientially, a digital game is more like a designed physical object than the more

64 Ibid., Chapter 11.

65 Roger Moseley, "Playing Games with Music (and Vice Versa): Ludomusicological Perspectives on Guitar Hero and Rock Band," Taking It to the Bridge, digital edition, eds. Nicholas Cook and Richard Pettengill, (Ann Arbor: Michigan UP, 2013).

66 Chris Crawford, The Art of Computer Game Design, digital edition, (Washington State University, 1997$), 13$.

67 Salen and Zimmerman, Rules of Play, 102. 
symbolic musical score. In a well-designed system, controls have a tendency to disappear allowing the player to become immersed in the simulated physics of the game world. Game composer David Kanaga contends that "all games have affordances; not all games have rules."68 Affordances represent what he calls the ecological ("ground of the household") aspect of games. Rules, on the other hand, represent what he calls the economic ("management of the household") aspect of games, and can be broken. He uses the example of game of basketball, in which the ball and hoop represent ecological aspects while the rules of the game constitute the economical. A traditional musical score might be considered primarily economical since it's based on conventional rules that are not strictly enforced by physical affordances and constraints. An equal consideration of both ecological and economical levels might fall under a larger category that Kanaga calls ecologonomy.

Since digital games are fundamentally dependent on affordances, they are often described as toys. Game scholar Brian Sutton-Smith asserts that, "[the video game] is the most complex toy every built and is vastly more responsive than any other toy ever invented."69 Implicit in this assertion is the acknowledgment that affordances in games are dynamic; they can be created or destroyed immediately and shaped continuously. Game designer Keith Bergun proposes a hierarchy of interactive forms which shows how all digital games

\footnotetext{
68 David Kanaga, "Intro to Ludic Ecologonomy (Pt. 1)," Wombflash Forest http://wombflashforest.blogspot.com/2015/04/intro-to-ludic-ecologonomy-pt-1.html [accessed 2016FEB01].

69 Brian Sutton-Smith, qtd. in Salen and Zimmerman, Rules of Play, 85.
} 
derive from toys, puzzles, and contests. ${ }^{70}$ Kanaga contributes to this model by showing his distinction of ecology and economy. (Figure 2) The notion of the digital game as toy is discussed further below.

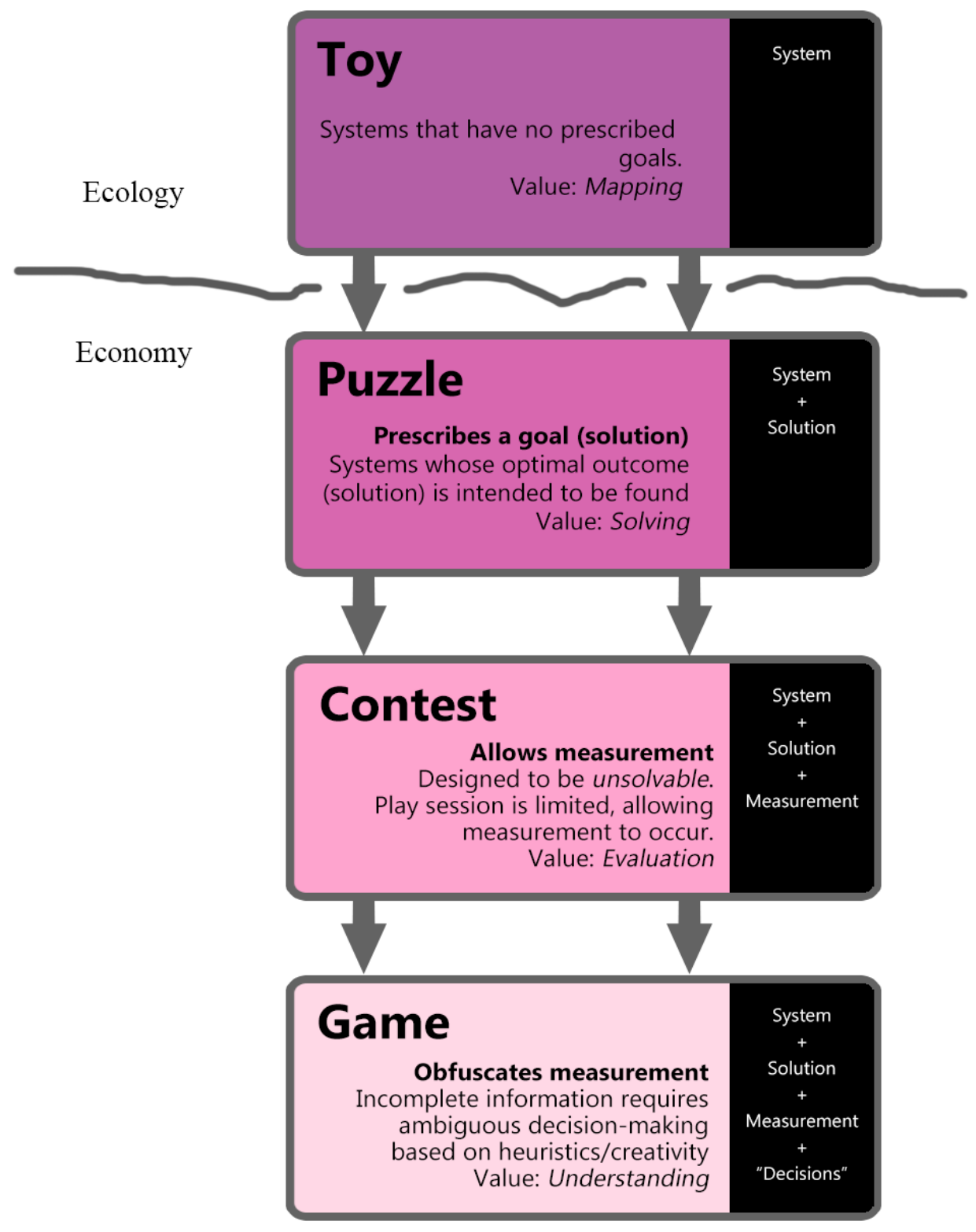

Figure 2. Bergun's hierarchy of interactive forms with alteration by Kanaga ${ }^{71}$

70 Keith Bergun, "The Four Interactive Forms," http://keithburgun.net/interactive-forms/ [accessed 2016FEB01].

71 Kanaga, "Intro to Ludic Ecologonomy (Pt. 1). 


\section{Isolating the Artifact}

In Music and Discourse, Jean-Jaques Nattiez invokes Jean Molino's semiotic tripartition as a model with which to parse out the elements of musicking. On the poietic level, music involves a "complex process of creation ... that has to do with the form as well as the content of the work." The esthesic level is characterized as a "complex process of reception ... that reconstructs a 'message."'72 The result of creation and the subject of reception is the neutral trace - a "symbolic form" that can "give rise to a complex and infinite web of interpretants." 73 Nattiez locates the "work's being in its dispersal between three spheres, in the interaction between its symbolic components as total musical face: as poietic strategies, a resultant trace, and esthesic strategies unleashed by that trace." ${ }^{.74}$ It is important to note that Nattiez doesn't consider his semiotic theory as a "science of communication" since communication is "only one of the possible results of the symbolic process. ${ }^{" 75}$ Communication happens when the producer and receiver share a common cultural context. This shared context for communication has been explained variously, including Eco’s semiotic “codes,"”7 Kofi Agawu's musical "topoi,"77 or Herbert Clark’s “common ground" for linguistic communication. ${ }^{78}$

72 Jean-Jacques Nattiez, Music and Discourse: Toward a Semiology of Music, (Princeton: Princeton UP, 1990), 17.

73 Ibid., 37.

74 Ibid., 70.

75 Ibid., 16-7.

76 Umberto Eco, A Theory of Semiotics, (Bloomington: Indiana UP, 1976).

77 Kofi Agawu, Music as Discourse: Semiotic Adventures in Romantic Music, (New York: Oxford UP, 2009).

78 Herbert H. Clark, Using Language, (Cambridge: Cambridge UP, 1996). 


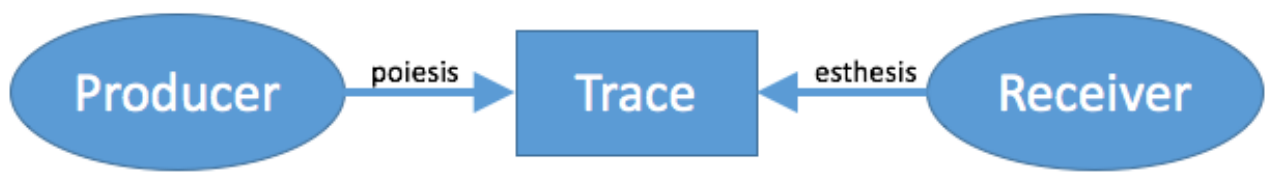

Figure 3. Basic semiotic tripartition

Rooted in Peircean semiotics, this model works well on a basic level for understanding the role of the musical score in relation to composers and performers. The score acts as a continual point of reference for the realization of the music rather than the music itself, subject to an infinite web of interpretations. As Eno states, "A musical score is a statement about organization; it is a set of devices for organizing behaviour toward producing sounds." 79 The semiotic model can be extended to account for traces that are designed to produce new traces. (Figure 4) In music, the score ("music as product") prescribes certain aspects of musical production ("music as process"). In some cases, like the open scores of the postwar era, they prescribe relatively little, allowing more player agency.

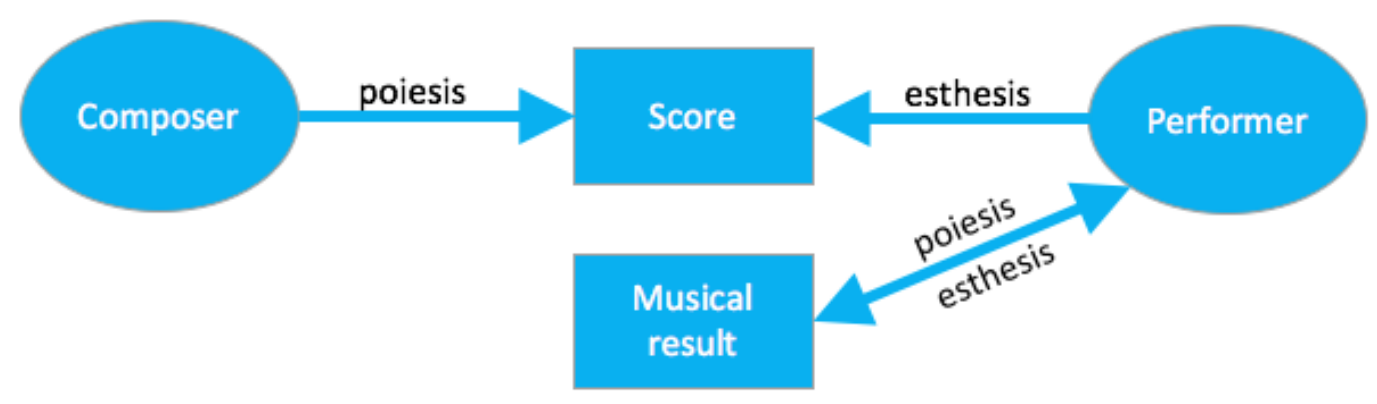

Figure 4. Extended tripartition for musical scores

79 Eno, "Generating and Organizing Variety in the Arts," Audio Culture, 226. 
The term 'trace' is useful but isn't sufficient for referring specifically to the musical score since it can denote different types of symbolic forms (e.g. the musical result). I shall henceforth refer to a material instance of the trace - i.e. a physical object created by poiesis that unleashes esthesic strategies - as an artifact. Furthermore, material traces that are designed to be performed shall simply be called performed artifacts. A musical score is an instance of a performed artifact.

Diana Taylor offers another taxonomy from the perspective of performance studies. She distinguishes between "the archive of supposedly enduring materials (i.e., texts, documents, buildings, bones) and the so-called ephemeral repertoire of embodied practical/knowledge (i.e. spoken language, dance, sports, ritual)." ${ }^{\prime 0}$ In this sense, the activities of composition and performance can be seen as categorically similar; both are facets of repertoire, which necessarily depend on embodied experiences and cultural concepts (i.e. vibrational affinity dynamics). On the other hand, instances of the archive (e.g. a performed artifact) are physically objective, even though their "value, relevance, or meaning" may change over time based on cultural context. ${ }^{81}$

80 Diana Taylor, The Archive and the Repertoire: Performing Cultural Memory in the Americas (Durham: Duke University Press, 2003), 19.

81 Ibid. 


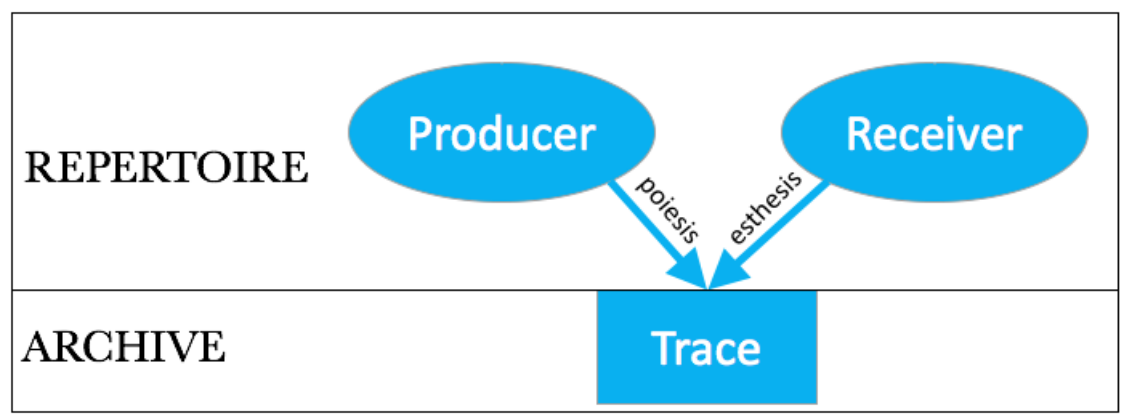

Figure 5. Taylor's archive-repertoire concept superposed over the semiotic tripartition

The Mechanics-Dynamics-Aesthetics (MDA) design model, proposed in a 2004 paper by Hunicke, LeBlanc, and Zubek, is similar to Nattiez and Taylor's models in that it separates material from behavior and describes the relationship between them. In this case, the trace is split into three parts. The game designer creates the mechanics of the game, which are made up of data and algorithms. The mechanics in turn determine the dynamics, which describes the run-time behavior of the game. Aesthetics describes the player's experience of the game. From the perspective of the player, the mechanics may be understood (or misunderstood) via the dynamics, which are understood through the aesthetics. (Figure 6) According to the authors, "thinking about the player encourages experience-driven (as opposed to feature-driven) design." 82 Their taxonomy of aesthetic goals is explored further in Chapter 3.

82 Robin Hunicke, Marc LeBlanc, Robert Zubek, "MDA: A Formal Approach to Game Design and Game Research," Proceedings of the Challenges in Games AI Workshop, Nineteenth National Conference of Artificial Intelligence, 2004. 


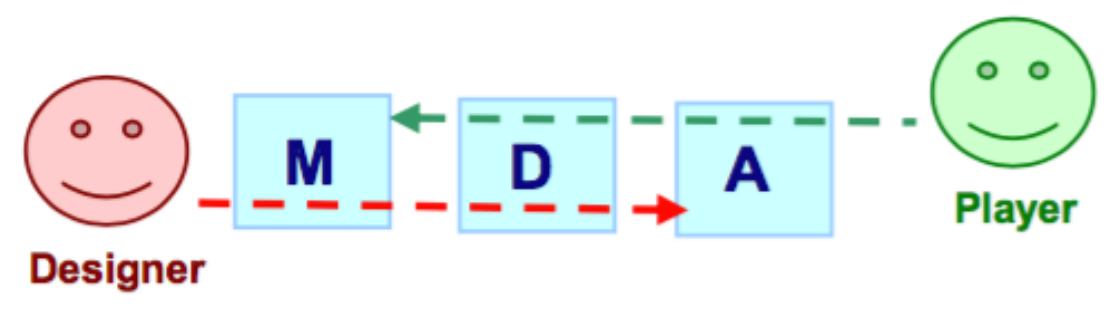

Figure 6. Mechanics-Dynamics-Aesthetics model of game design ${ }^{83}$

Like score-based musical performance, we understand the experience of playing a digital game to be contingent on choices that are made in relation to the properties of an artifact (i.e. esthesis). Yet despite this basic functional similarity, digital games are interactive performed artifacts; their dynamics change as a result of player action, and such changes provide important information to the player. Furthermore, games typically act as the sound source instead of the player, though players can of course make their own sounds and are at times expected to do so.

Clearly, the semiotic model for games is a bit more complex since the artifact is not only interactive but also directly involved in poiesis. (Figure 7) Musician and sound-artist Norbert Herber explores a similar model for what he describes as the compositioninstrument, which blurs conventional distinctions between each constituent part. It is "a work that can play and be played simultaneously. A composition-instrument is not a specific piece of music or interactive work in itself but a means of approaching any work

83 Ibid. 
where music can be created and transformed." ${ }^{44}$ He explicitly relates this concept to three musical traditions mentioned above: experimental (i.e. open works), improvisatory, and generative. Somewhat similar to the extended tripartition for digital games (Figure 7), his model describes the flow of information as perturbations that echo throughout a three-component system, affecting each and every other interconnected component. (Figure 8)

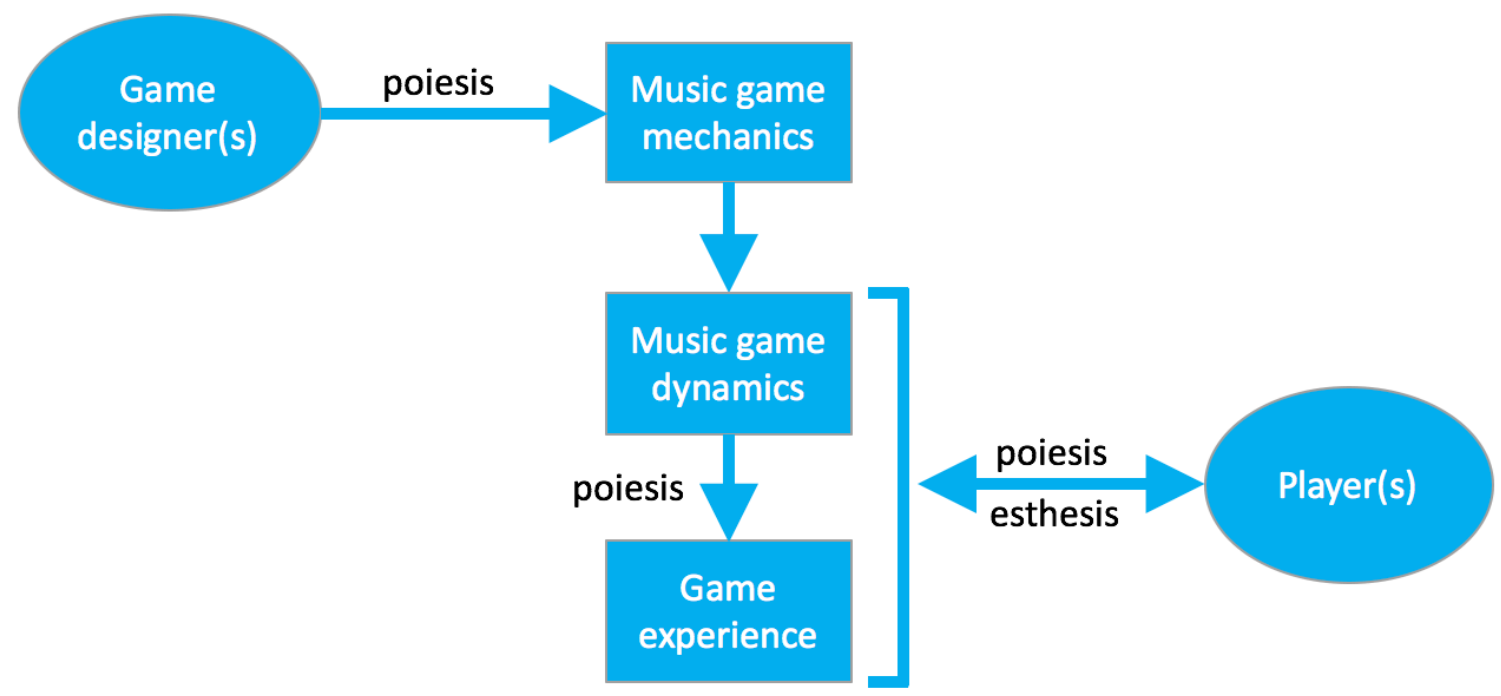

Figure 7. Extended tripartition for digital games

84 Norbert Herber, "The composition-instrument: emergence, improvisation and interaction in games and new media," From PacMan to Pop Music: Interactive Audio in Games and New Media, Ed. Karen Collins, (Farnham, Surrey, GBR: Ashgate Publishing Group, 2008), Chapter 7, pp. 104. 


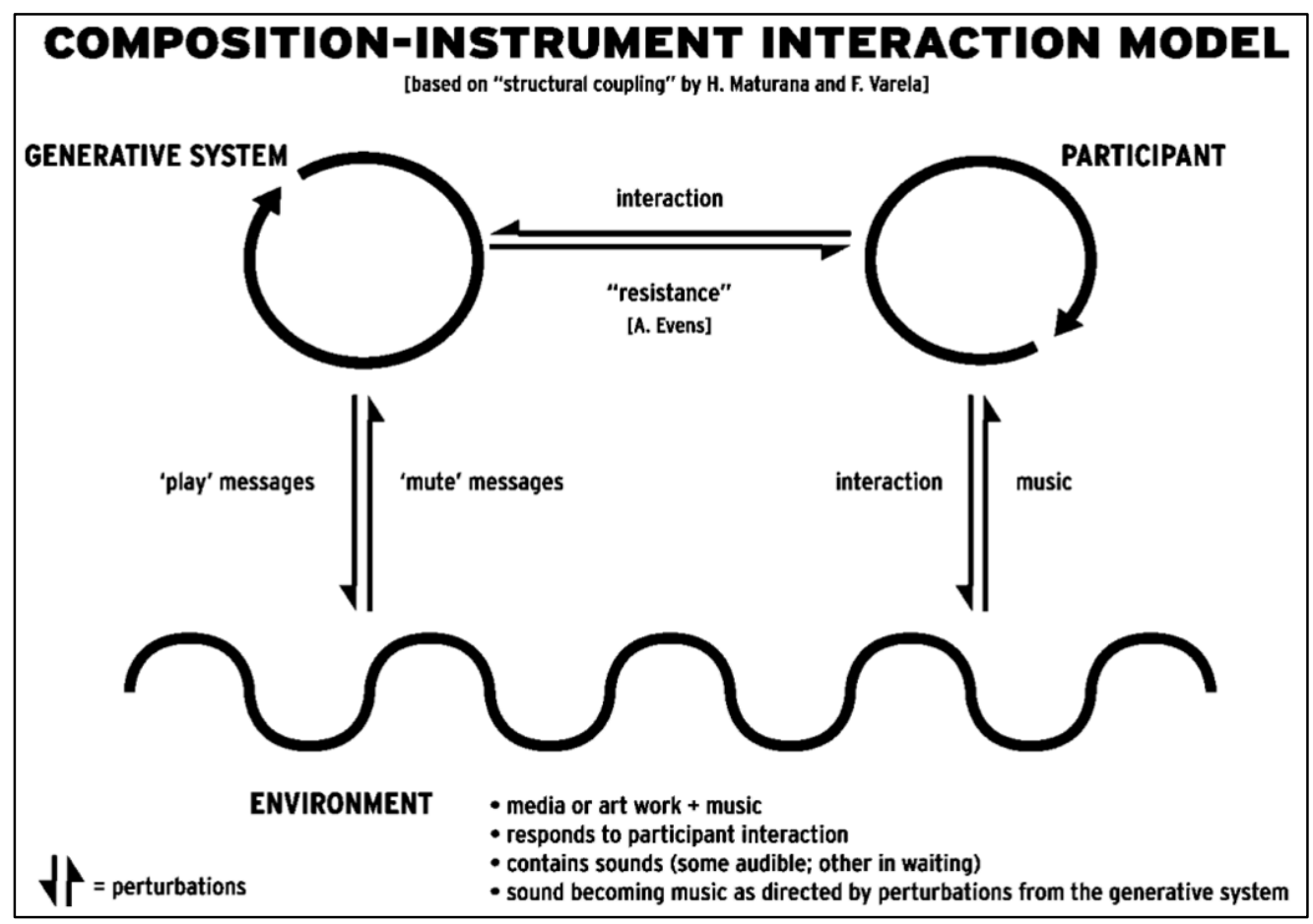

Figure 8. Herber's model of the composition-instrument

Immersion is an important aspect of the way a player experiences a game. Laura Ermi and Frans Mäyrä explore immersion in their paper titled, "Fundamental Components of the Gameplay Experience: Analysing Immersion." 85 They recognize three types of immersion that digital game players experience: 1) sensory immersion, or the audiovisual execution of the game, 2) challenge-based immersion, which relates to the balance of challenge to player ability, and 3) imaginative immersion, which relates to characters and story elements. Figure 9 below shows their model for how immersion functions.

85 Laura Ermi and Frans Mäyrä, "Fundamental Components of the Gameplay Experience: Analysing Immersion," Changing Views: Worlds in Play. Selected Papers of the 2005 Digital Games Research Association's Second International Conference, Eds. Suzanne de Castell and Jennifer Jenson, 15-27. 


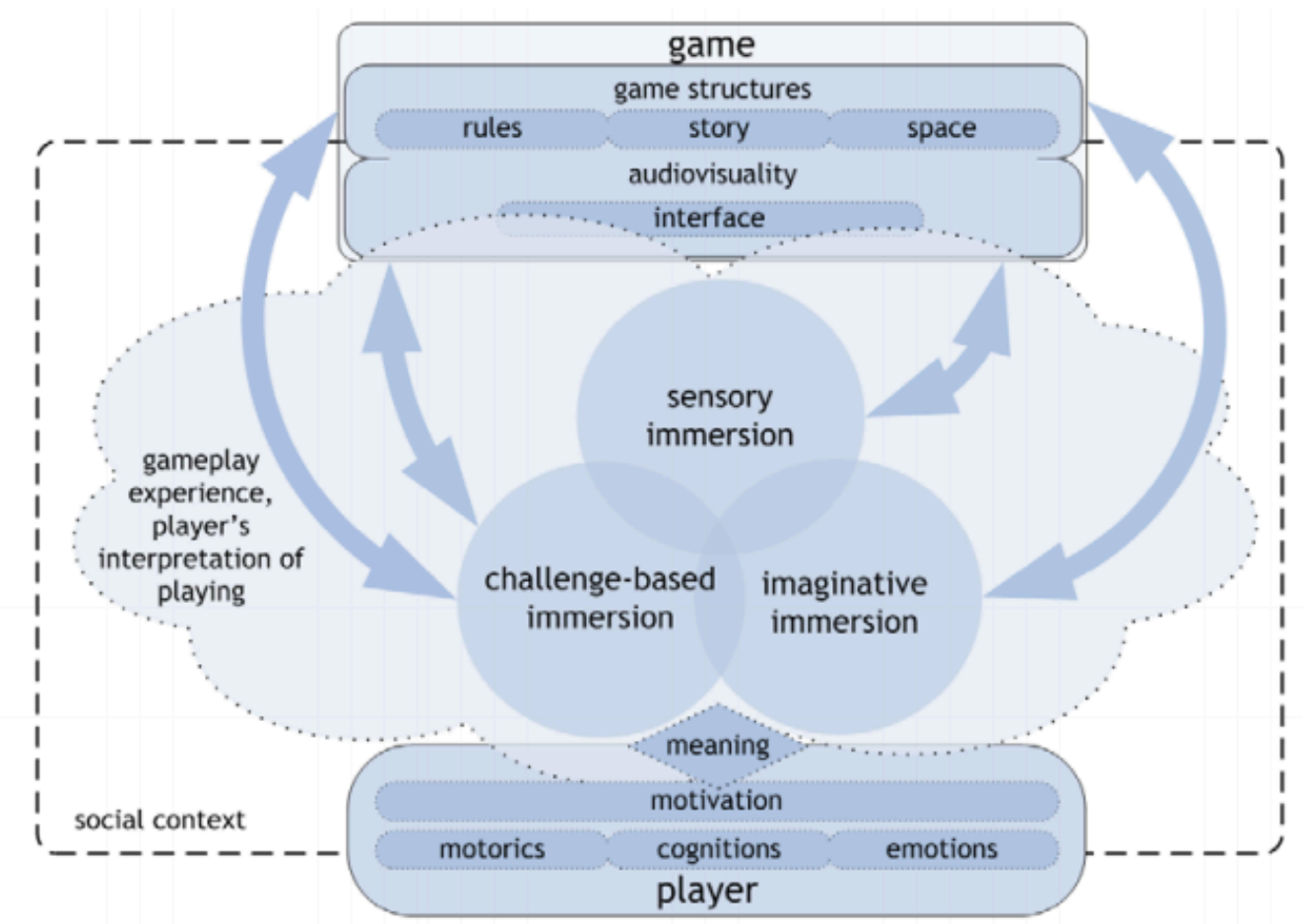

Figure 9. Ermi and Mäyrä's model of immersion ${ }^{86}$

\section{Object-Oriented Ontology}

While object-oriented ontology, or OOO, is a deep topic which extends well beyond the scope of this dissertation, some of its key concepts apply to discussions of dynamic artifacts. Coined by philosopher Manuel DeLanda ${ }^{87}$ and developed by others such as Levi Bryant ${ }^{88}$ and Ian Bogost ${ }^{89}$, OOO proposes a flat ontology, in which the human-world

Ibid., 8.

87 Manuel DeLanda, Intensive Science and Virtual Philosophy, (London: Continuum, 2002), 47.

88 Levi Bryant, "Flat Ontology," Larval Subjects, https://larvalsubjects.wordpress.com/2010/02/24/flatontology-2/, [accessed 2016JAN30].

89 Ian Bogost, Alien Phenomenology, or What It's Like to Be a Thing, digital edition, (Minneapolis: Minnesota UP, 2012). 
relationship is not privileged (as it is in Meillassoux's correlationism) but merely one of many potential relationships between all things. Moreover, it allows for the subjectless object to exist- "an object that is for-itself rather than an object that is an opposing pole before or in front of a subject." 90

In his dissertation on "Ontological Toys," Jordan Bartee explains that "the point [of OOO] is not to reduce the status of human beings, but to extend that status to other beings, taking all objects seriously as real autonomous actors." 91 He applies the lens of OOO to virtual objects in digital games like the sprites produced by his Commodore 64 . According to Bartee, "the uniqueness of computer-technological objects is not in their ontological structure, but in the way they call explicit attention to that structure through their visible complexity and multidimensionality."92 He also applies OOO to his own complex creations, which he refers to as ontological toys. Ontological toys are instruments, which he defines as "[human]made systems that enact possibilities from limitations through work and play. To the extent that an instrument is used in play, it may be called a toy; to the extent that it is used in work, it may be called a tool."93 Via game designer Chaim Gingold's master's thesis ${ }^{94}$, Bartee distills four essential features of ontological toys: 1) abstraction, which refers to visual presentation and toy behavior, 2) broadness, which

90 Levi Bryant, The Democracy of Objects, (Ann Arbor: Open Humanities Press, 2011$), 19$.

91 Jordan Bartee, "Ontological Toys," (PhD diss., Brown University, 2014), 27.

92 Ibid., 32.

93 Ibid., 57.

94 Chaim Gingold, "Miniature Gardens \& Magic Crayons: Games, Spaces, \& Worlds," (master's thesis, Georgia Institute of Technology, 2003), http://levitylab.com/cog/writing/thesis/ [accessed 2016JAN30]. 
concerns the relationship between the microscopic and macroscopic levels of the toy, 3)

freedom and 4) authorship. Abstraction relates to feelings of immersion; more specifically, "ontological toyness emerges out of simulations in which there is tension between a high degree of imaginative immersion and a low degree of sensory immersion."95 Freedom and authorship are discussed more closely in the following chapters.

95 Bartee, “Ontological Toys,” 66. 


\section{Ghapter 2: Player Experience}

\section{Memory and Perception-Action}

Music notation depends heavily on memory in different ways. On a basic level, realizing musical notation requires the ability to recall mappings of symbol to sound and/or action.

This "Western craziness" extended the transmissions of works over space and time, and

served a mnemonic purpose, as described by composer Jacques Charpentier:

When at the end of the Middle Ages, the Occident attempted to notate musical discourse, it was actually only a sort of shorthand to guide an accomplished performer, who was otherwise a musician of oral and traditional training. These graphic signs were sufficiently imprecise to be read only by an expert performer and sufficiently precise to help [them] find [their] place if, by mishap, [they] had a slip of memory. Consequently, as we see, it was not a question of precise notation but rather a mnemonic device in written symbols. ${ }^{96}$

Additionally, memory is involved when performers construct conceptual models of a musical work, which are informed by rules - e.g. performance instructions, notational symbols, and the cultural context of the piece - as well as the affordances and constraints of the instrument. In a performance, the way that performers react to sound relies, at least in part, on any conceptual models they have constructed. Furthermore, performances of a particular work contribute to the overall context surrounding that work, which of course informs subsequent performances. According to Diana Taylor,

96 Jacques Charpentier, qtd. in Bailey, Improvisation, 59. 
"performances function as vital acts of transfer, transmitting social knowledge, memory, and a sense of identity through reiterated, or what Richard Schechner has called 'twicebehaved behavior."' In other words, performance is not only the act to be studied but also the "methodological lens that enables scholars to analyze events as performance."97

Memory is crucial to the experience of playing digital games as well. On the surface level, players need to retain information about controller configurations and game structures (e.g. levels, movement patterns, etc.) to succeed, which is supported by strong conceptual models via proper interface design. These patterns may be idiosyncratic but usually conform to common conventions, such as the mapping of the most common game functions (e.g. jump) to the most easily accessible button (e.g. X on a Playstation controller). Additionally, cultural context often influences a player's gaming experience. For example, digital game genres regularly inform player expectations and permit "aesthetic appreciation from the players literate in these conventions ... by streamlining the functional aspects of a particular game."98

Prominent theories on how memory informs perception and action are rooted in early philosophical inquiries. In his Matière et mémoire, Henri Bergson posits that, we are constantly creating or reconstructing. Our distinct perception is really comparable to a closed circle in which the perception-image, going towards the mind, and the memory-image, launched into space, careen the one behind the other.... Reflective perception is a circuit, in which all the

97 Taylor, The Archive and the Repertoire, 2-3.

98 Dominic Arsenault, "Video Game Genre, Evolution and Innovation," Eludamos. Fournal for Computer Game Culture, (Vol. 3, No. 2, 2009), pp. 149-176. 
elements, including the perceived object itself, hold each other in a state of mutual tension as in an electric circuit, so that no disturbance starting from the object can stop on its way and remain in the depths of the mind; it must always find its way back to the object whence it proceeds. ${ }^{99}$

This distinction between the "perception-image" and the "memory-image" is also described as real object versus virtual object, respectively. ${ }^{100}$ For Bergson, the utility of memory is solely for directing action, bridging the body and spirit. The memory-image or virtual object is a product of the continuous bi-directional flow between "pure memory" and perception. ${ }^{101}$

However, for Bergson, as with Zeno of Elea, thinking about memory in terms of fixed images is paradoxical since time and movement are actually continuous and indivisible. ${ }^{102}$ He states, "the truth is that this independent image is a late and artificial product of the mind." 103 David Trippett suggests that the paradox can be better understood by relating it to Hegel's use of the terms Erinnerung and Gedächtnis, though the exact definition of these terms varies depending on the source. ${ }^{104}$ While Trippett translates Erinnerung to "recollection" and Gedächtnis to "representation," a slightly different and perhaps more thorough interpretation is given by M. J. Inwood:

Hegel takes Erinnerung to be, not primarily recollection, but the internalization of a sensory intuition as an image; the image is abstracted from the concrete spatio-temporal position of the intuition, and given a

99 Henri Bergson, Matter and Memory, trans. Nancy M. Paul and W. Scott Palmer (New York, 1911 [orig. French 1896]), 126-7.

100 Ibid., 167.

101 Ibid., 114.

102 Ibid., 249-252.

103 Ibid., 214.

104 David Trippett, "Composing Time: Zeno's Arrow, Hindemith's Erinnerung, and Satie's Instantanéisme," Fournal of Musicology, vol. 24 (2007): 522-80. 
place in the intelligence (which has its own subjective space and time). But the image is fleeting, and passes out of consciousness. The imagination is thus needed to revive or reproduce the image. The imagination is successively reproductive, associative, and productive or creative.

However creative the imagination may be, its images are still images of intuited objects. Liberation from intuition and image is provided by Gedächtnis. Hegel associated this with thought: the past participle of denken ('to think') is gedacht ('(having been) thought'), so that Gedächtnis has the flavour of 'having-been-thoughtness'. Hence Gedächtnis, though it precedes thought itself in Hegel's account, is thought-memory, and, since thinking, on Hegel's view, involves language, verbal memory. ${ }^{105}$

Inwood's definition provides a clearer explanation of the paradoxical dichotomy of real, temporal memory (Erinnerung), which is often unconscious and fleeting, and the memory-image that we willfully command (Gedächtnis). Like Inwood, Jacques Derrida asserts that Gedächtnis represents freedom from intuition, i.e. "thinking memory," which is necessary for creating art. ${ }^{106}$

Crucial to Bergson's position on memory is the importance of both temporal perception and timeless memory-images working together as a whole to create action. He rails against associationism, which explains the selection of memories by some "mysterious attractions" between atoms of memory-images and perceptions. Instead, Bergson submits that we perceive resemblance before we perceive individual parts, and that "our entire personality, with the totality of our recollections, is present, undivided within our actual perception." 107

\footnotetext{
105 M. J. Inwood, A Hegel Dictionary (Wiley-Blackwell, 1992), 188.

${ }^{106}$ Jacques Derrida, Memoires for Paul de Man, revised edition (Columbia UP, 1989), 67.

107 Bergson, Matter and Memory, 214.
} 
Interestingly, Bergson's philosophical notion of integrated perception-action is supported by our current understanding of the manner in which the human brain processes memories and perceptual information. Neuroscientist Joaquin Fuster describes working memory and its relationship to the perception-action cycle as a multi-modal, two-way process. His findings support the so-called "network paradigm," which asserts that memory and knowledge are "represented in widely distributed, interactive, and overlapping neuronal networks of the cerebral cortex," as opposed to the "modular paradigm," in which "cognitive functions are localized in discrete brain regions, each dedicated to a different function (attention, perception, memory, language, and intelligence)." Fuster's model essentially posits that the pursuit of a goal involves "mediation of cross-temporal contingencies between percepts and actions." 108 In other words, sensations, concepts, memories, and actions are all inextricably interrelated. (Figure 10)

\footnotetext{
${ }^{108}$ Joaquin Fuster, "Distributed Memory and the Perception-Action Cycle," Recorded lecture from Conference on Brain Network Dynamics held at the University of California at Berkeley on January 26-27, 2007, https://archive.org/details/Brain_Network_Dynamics_2007-13-Joaquin_Fuster [accessed on 2015SEPT09].
} 


\section{Executive memory Perceptual memory}

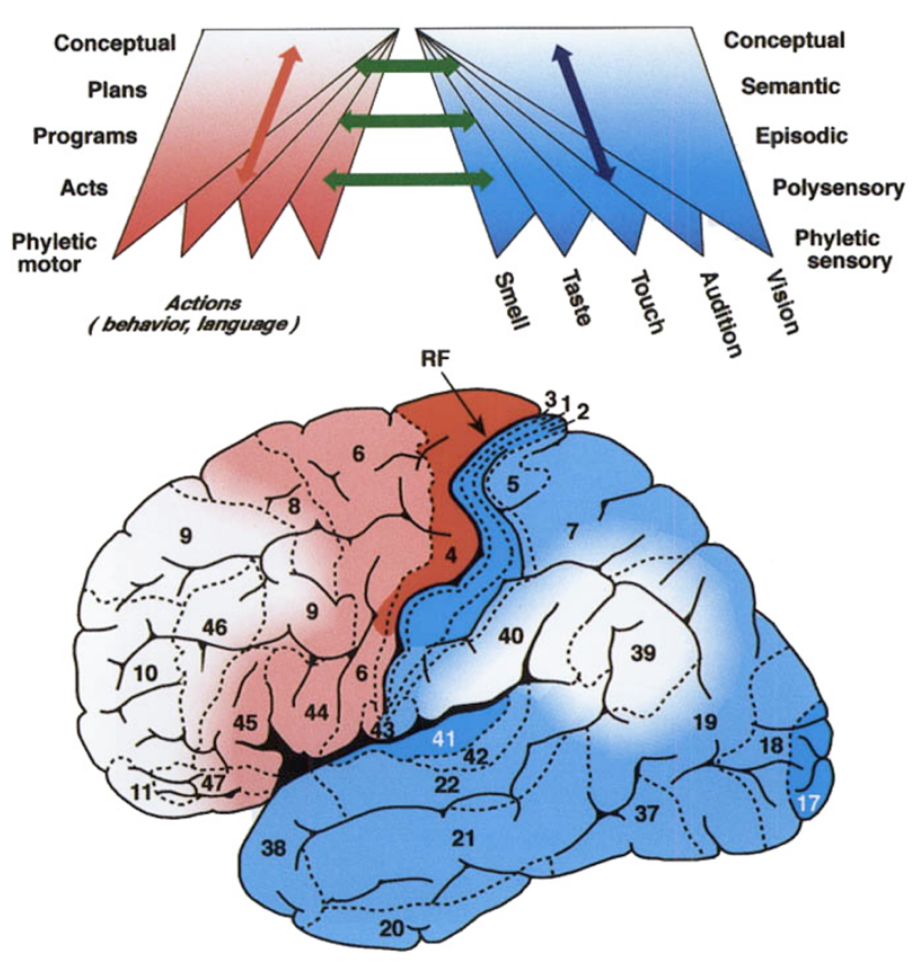

Figure 10. Fuster's multimodal two-way model of perception-action ${ }^{109}$

Initially coined by Gilles Fauconnier and Mark Turner, conceptual blending involves two input mental spaces (e.g. Gedächtnis and Erinnerung) to yield a third blended mental space. ${ }^{110}$ Cognitive scientist Edwin Hutchins builds on the notion of conceptual blending by asserting that material anchors can stabilize such a blend. For example, the perception of people standing in a particular formation and the material anchor of a line combine to yield the concept of a queue. (Figure 11)

${ }^{109}$ Joaquin Fuster, Cortex and Mind: Unifying Cognition, (New York: Oxford UP, 2003), Color Plate 1.

110 Gilles Fauconnier and Mark Turner, The Way We Think, (New York: Basic Books, 2002). 


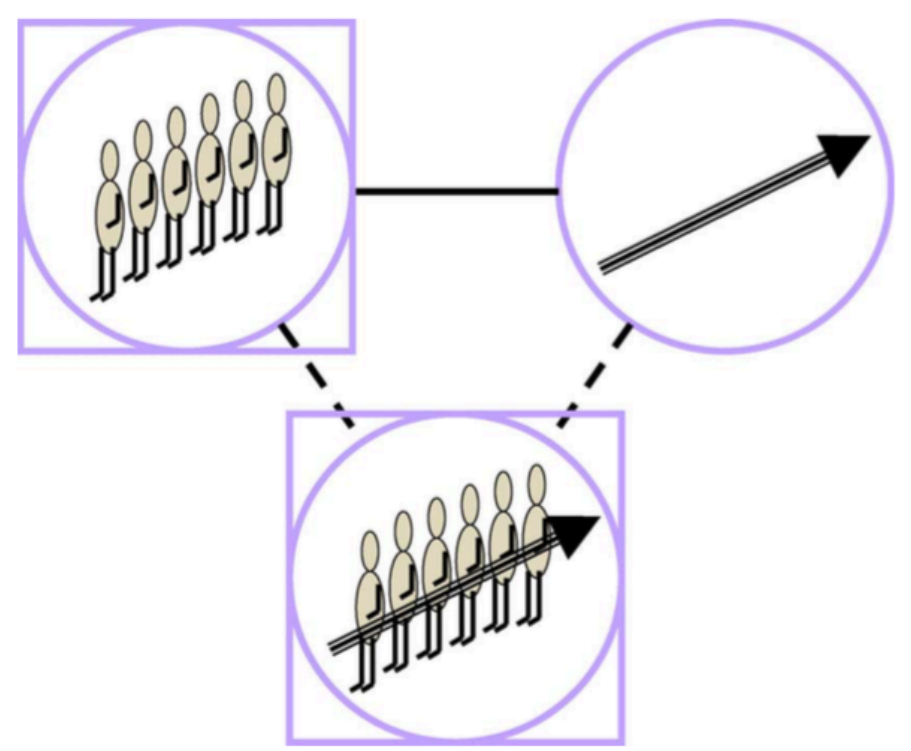

Figure 11. Hutchins's example of a material anchor stabilizing a conceptual blend

Kenny Chow and D. Fox Harrell elaborate on Hutchins's material anchor concept by considering animated anchors as they exist in dynamic images (e.g. film, digital games). In their words, "animated images work as elastic anchors for sensational, visceral, and elaborative conceptual blends because they more readily engage our embodied sensations through the coupling of perceived action and motor knowledge."111 One example they provide is the animation that accompanies the act of maximizing or minimizing a window in the Macintosh OS X operating system. As the window stretches and twists, it resembles a genie emerging from or receding into a lamp. This animation creates the conceptual blend of a "powerful genie application serving at your wish ... [performing a] spectacular magical function."112

\footnotetext{
111 Kenny Chow and D. Fox Harrell, "Material-based Imagination: Embodied Cognition in Animated Images," Proceedings of the Digital Arts and Culture Conference, 2009. 112 Ibid.
} 
Case Study: Paul Turowski, Study No. 2/2.5 (2011)

Study No. 2 and Study No. 2.5-henceforth collectively referred to as Study 2.x-were directly inspired by these notions of memory and perception-action. The animated digital score, which is visible to the audience via video projection, is generated by a computer in real-time (i.e. during the performance) and interpreted improvisationally by four performers. In Study No. 2, the ensemble consists of flute, bass clarinet, violin, and cello; Study No. 2.5 features four electric guitars with effects pedals. Each player reads one quadrant of the screen. At various times throughout the performance, pitch sets of two to three notes are displayed on three different shapes: circles, triangles, and squares. (Figure 12) When a pitch set is displayed on a shape, nothing is played at that point; the performer must only remember the pitch set, creating a mnemonic association between set and shape.

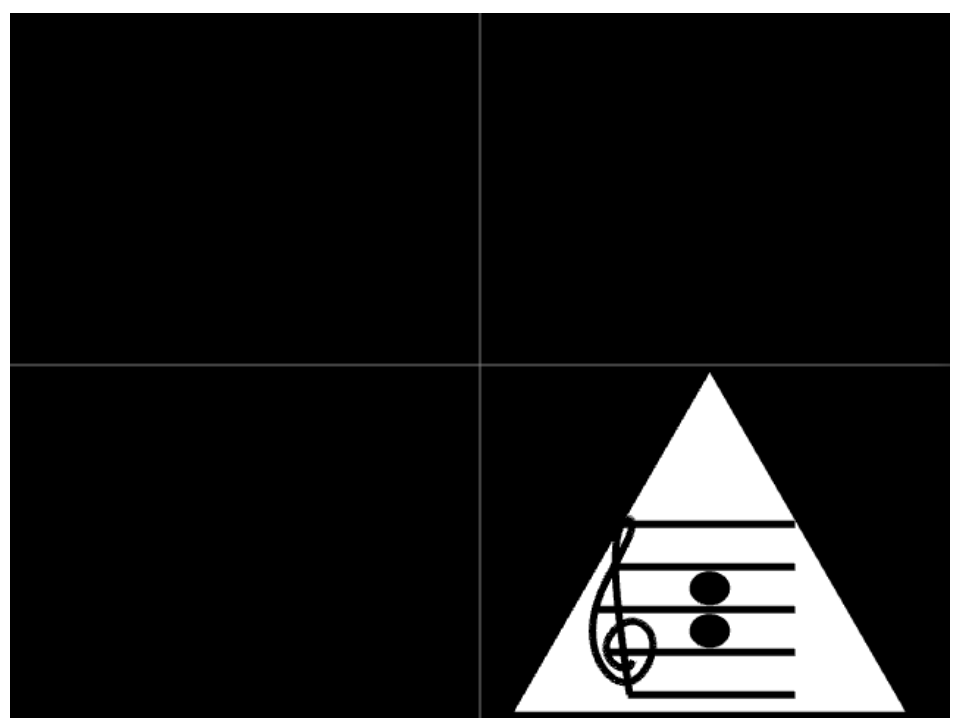

Figure 12. Turowski, Study No. 2.x. A pitch set is associated with a shape 
Once this key disappears, shapes (without musical notation) enter the screen from beyond the border of the display. (Figure 13) Pitch distribution as well as shape origin, direction and speed are all determined by random number generators in the Max programming environment. ${ }^{113}$ Players then improvise a melodic motive using the pitches that are associated with the shapes present in their quadrant. Loudness is determined by the size of the shape on the screen. Since the shapes all gradually reduce in size over time, the players continually apply decrescendi to the motives they create. Furthermore, the shapes gravitate toward the center creating a sense of perspective.

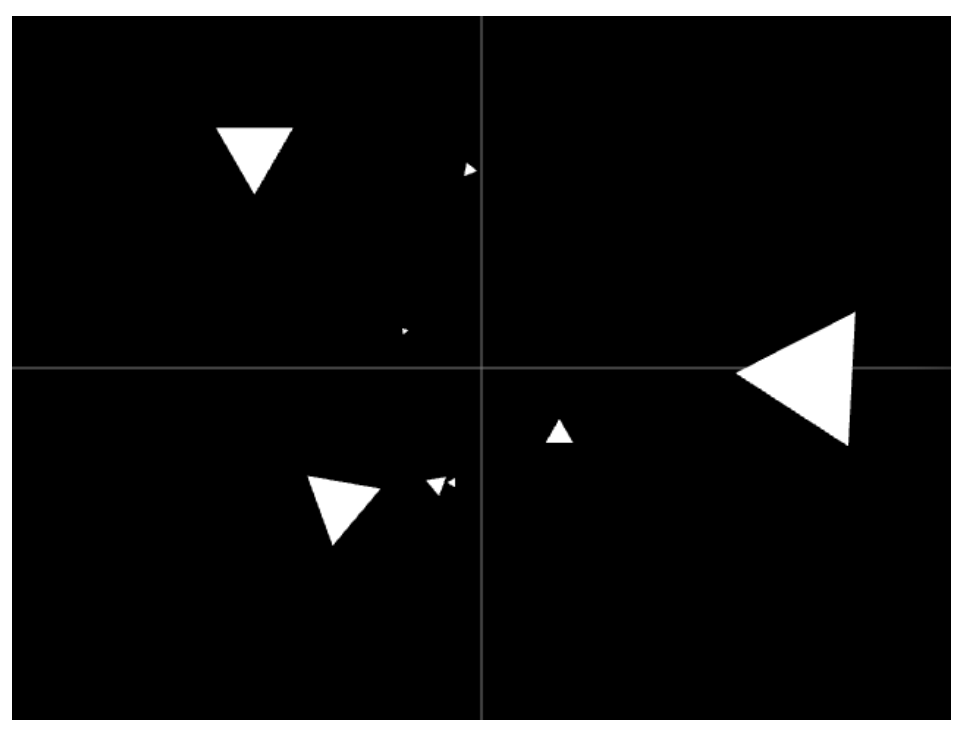

Figure 13. Turowski, Study No. 2.x. Shapes enter and recede into background

The manner of playing is also affected by wave-like patterns of color that emanate from

${ }^{113}$ Henceforth, "random" shall denote the pseudo-random functions commonly used by most computersi.e. algorithms that are influenced by a unique numerical seed, such as the time of day and date. 
center of the screen. (Figure 14) This visual effect generally indicates agitations of timbre. For example, a cellist might bow with increasing force or a guitarist might gradually incorporate a tremolo effect as the patterns become brighter and more complex.

Electronic sounds accompany the color waves and serve as a pitch reference for the players.

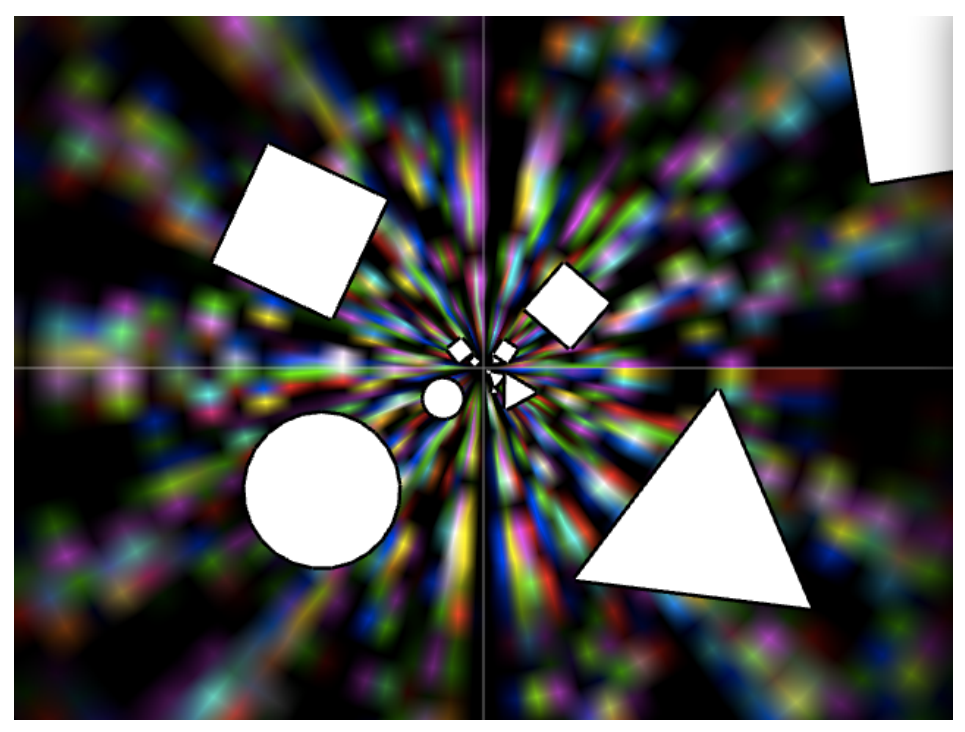

Figure 14. Turowski, Study No. 2.x. Waves of color indicate agitation of timbre

Bergson's notion of a unified duality was one of the central catalysts in the revision of Study No. 2. As mentioned above, the bi-directional flow is depicted in the video score as shapes move away from the viewer while the waves of color move toward. This unceasing motion might be considered Erinnerung, not only symbolically but practically as the performers must react in real-time to changes in the visual data. By contrast, pitch sets (i.e. representational data) are displayed statically and are more characteristic of Gedächtnis. They are revealed over time and are internalized through Erinnerung, but 
once internalized the pitch sets are transformed into thought-memory or conceptual blends, to be internally manipulated. These thought-memories guide the performers' reactions in a complex network of perception and action, within which Erinnerung and Gedächtnis operate inseparably. Also, added in Study No. 2.5, the color waves have intermingled within them single static frames which pulse at regular intervals. These further depict the memory-image, situated between the axes of pure memory and perception. Like the example of the OS X window, animated images serve as elastic anchors that establish conceptual blends for the performer.

In Study $\mathcal{N}_{0} .2$, any given shape was given multiple pitch sets over the duration of the piece. This not only made the task of remembering difficult for the players but seemed to confuse the conceptual metaphor. In Study $\mathcal{N}_{0}$. 2.5, shapes have fixed pitch sets for the entire duration of a single performance, which served to establish stronger conceptual blends - both practically and metaphorically. (The root notes and the allocation of sets to quadrants differ from performance to performance.) This required a revision of the pitch sets themselves to create meaningful aggregates in service of the overall concept. In the beginning of the revised version, performers are given the same shapes at the same time, and the resulting combination of pitch sets creates atonal harmonic relationships. (Table 2) Over time, different combinations of shapes are introduced and harmonic relationships increasingly become more consonant. As tonality emerges toward the end of the piece (Table 3), shapes, color patterns, and sounds all fuse together as one. 


\begin{tabular}{|l|c|c|c|c|c|c|c|}
\hline pitch set 1 & 0 & 2 & 3 & $6^{*}$ & 7 & 9 & 11 \\
\hline pitch set 2 & 0 & 2 & 4 & 5 & 7 & 9 & 11 \\
\hline pitch set 3 & 0 & 2 & $5^{*}$ & $6^{*}$ & 7 & $10^{*}$ & 11 \\
\hline pitch set 4 & 0 & 2 & 3 & 5 & 7 & 9 & 11 \\
\hline
\end{tabular}

Table 1. Turowski, Study 2.x. Pitch classes

(* denotes pitches altered from major/minor)

\begin{tabular}{|l|c|c|c|}
\hline & & & \\
\hline Player 1 & $\{2,7,11\}$ & $\{0,9\}$ & $\{3,6\}$ \\
\hline Player 2 & $\{3,8,10\}$ & $\{2,5\}$ & $\{0,7\}$ \\
\hline Player 3 & $\{0,1,6\}$ & $\{4,11\}$ & $\{5,8\}$ \\
\hline Player 4 & $\{0,4,9\}$ & $\{6,8\}$ & $\{2,11\}$ \\
\hline
\end{tabular}

Table 2. Turowski, Study 2.x. Set ordering in the first half

\begin{tabular}{|l|c|c|c|}
\hline & Imaj7/vi & V7 (m9) & Ger+6 (M2, M4) \\
\hline Player 1 & $\triangle_{\{0,9\}}$ & $\square_{\{2,7,11\}}$ & $\bigcirc_{\{3,6\}}$ \\
\hline Player 2 & $\bigcirc_{\{0,7\}}$ & $\triangle_{\{2,5\}}$ & $\square$ \\
\hline Player 3 & $\triangle_{\{4,11\}}$ & $\bigcirc_{\{5,8\}}$ & $\square_{\{0,1,6\}}$ \\
\hline Player 4 & $\square_{\{0,4,9\}}$ & $\bigcirc_{\{2,11\}}$ & $\triangle_{\{6,8\}}$ \\
\hline
\end{tabular}

Table 3. Turowski, Study 2.x. Set ordering in the second half

As previously mentioned, Diana Taylor regards performance as the ephemeral carrier of cultural, embodied knowledge. It is a facet of the repertoire, as opposed to the archive of 
tangible materials, such as written text. ${ }^{114}$ Cultural parcel in Study 2.x is conveyed via the improvisation of the players. Within certain boundaries (including social interaction, which is discussed below), the musicians are free to shape what they play, and their choices are surely guided by past experiences. For example, each person may have different preferences regarding tone, articulation, or style. This embodied knowledge is Gedächtnis, and is transmitted between musicians as well as to the audience. The live nature of the piece, the ephemerality, requires acute perceptual awareness at all times (Erinnerung) in order to allow this communication to happen.

In contrast to Bergson's views about the personal nature of memory, Maurice Halbwachs claims that we necessarily must think and remember as a part of some social framework. "Original" memories are "explained by the fact that they are found at the intersection of two or more series of thoughts, connecting them in turn to as many different groups." 115 This concept of collective memory inspired a particular revision to the performance instructions for Study №. 2.5. In the newer version, players are asked to listen carefully to what the other members of the ensemble are playing and respond accordingly. If a shape enters one player's quadrant from another quadrant, that person should take on some characteristics of the motivic content from the originating quadrant. In other words, they are to shape their musical "memory" in the context of someone else's perspective. The precise manner and extent of this co-opting is left to the performer. This revision is

114 Taylor, The Archive and the Repertoire, 19.

115 Maurice Halbwachs, The Collective Memory, trans. Francis J. Ditter, Jr. and Vida Yazdi Ditter (New York: Harper \& Row, 1980 [orig. French 1950]), 40. 
supported by the use of one common score shared by all players rather than individual parts on separate screens (as was the case with Study No. 2). Additionally, the musicians are asked to always be mindful of the total sound at any given time and to subtly adjust their playing accordingly. Overall, Study 2.x is designed to produce an organic evolution of musical content - i.e. the germination of seeds in a sonic garden.

\section{Musical Intention}

A musician is commonly defined as someone who plays an instrument. Implicit in such a definition is the notion that anyone who makes sound is a musician. While this notion may be interesting philosophically, I would argue that it reduces the utility of the word (everyone makes sound) and negates the importance of intention. Perhaps a more useful definition of musician is one who makes sound musically, which includes making intentional musical choices. Of course, existing sounds (e.g. sounds from the environment) can be considered musical even if one is not playing these sounds. In such a case, the listener is the musician in that they are making the choice to consider these sounds musical. Even the decision to avoid intention in a composition (as with Cage) is still a decision about sound and the arrangement of sound. As such, my definition of musician precludes any incidental sound-making, such as the sound produced by a game while a player focuses solely on a non-musical objective. However, the role of musician can be easily adopted as soon as musical intention becomes part of the activity. 


\section{Music-Based Digital games}

In a paper by game scholars Martin Pichlmair and Fares Kayali, and later in Kayali's dissertation, music-based digital games (sometimes referred to simply and somewhat ambiguously as 'music games') are classified as being either rhythm games, electronic instrument games, or musical puzzles or challenges. ${ }^{116}$ In rhythm games, players must match predetermined tempi within a certain tolerance. Instrument games allow the player to make particular sounds with fewer or no temporal constraints. Musical puzzles or challenges happen within the context of a regular game, such as the use of the ocarina in The Legend of Zelda: Ocarina of Time. According to Kayali, rhythm games are more aligned to the experience of playing a game, whereas instrument games are more aligned with the experience of playing music. He concedes that games may feature both, but argues that "the fixed structure of rhythm games contradicts the sandbox gameplay of instrumental games."117

Pichlmair and Kayali's typology is useful, though I would suggest a few modifications. First, it seems that rhythm games are actually part of a larger category that I would call the musical accuracy game, which could be based on any musical parameters, not just rhythm. For example, in the music game series SingStar, singers match pitch as well as rhythm. Second, I find the category of instrument game to be slightly confusing since

116 Martin Pichlmair and Fares Kayali, "Levels of Sound: On the Principles of Interactivity in Music Video Games," Situated Play, Proceedings of DiGRA Conference, 2007.

117 Fares Kayali, "Playing Music: Design, Theory, and Practice of Music-based Games," (PhD diss., Vienna University of Technology, 2008). 
accuracy games often use instrument-shaped controllers in a performative setting.

Instead, the term musical sandbox game seems to be more accurate in describing their unique quality. Third, I would suggest that a musical puzzle or challenge is primarily distinguished by context and could actually be described as a sub-class of the other two categories. An important distinction between musical accuracy and musical sandbox games is that in the former success-failure is objective while in the latter success-failure is subjective. (Success-failure is discussed further below.) It is also important to note that these categories are not discrete but rather poles on a continuum like composition and improvisation. (See Chapter 1.)

Kayali also includes a taxonomy of features common to digital music-based games that facilitates further classification. ${ }^{118}$ Some of the most relevant to the present discussion include synesthesia, quantization, and audio input, sound agents, and active score. He asserts that most games don't focus on any particular feature but rather a combination of multiple approaches. ${ }^{119}$

\section{Gamers as Musicians}

Since the first commercial arcade game to feature sound (Computer Space, 1971), music has

\footnotetext{
118 While Pichlmair and Kayali refer to these factors as "principles," they seem to be less fundamental and essential than such a term would suggest. For example, a music game that lacks sound agents seems to be no less a music game.

119 Kayali, "Playing Music," 106.
} 
continually influenced player understanding and behavior. ${ }^{120}$ In her guide to music composition for digital games, Winifred Phillips discusses various functional roles of music in games, from music that establishes a state of mind to more semantic manifestations like "stingers," which "alert the player to a change in the current state of gameplay."121 Collins cites various modes of listening that players engage when playing games including causal listening ${ }^{122}$, in which players focus on recognizing the source of a sound, signal listening, in which players anticipate a cue, and retentive (memory) listening, which is perhaps most clearly demonstrated by the early sound-based electronic game Simon.

While many early theories and analyses of games are ocularcentric, Collins contends that

the stakes for players' involvement, interpretation, and therefore attention are much higher in games, so they listen more actively and employ different modes of listening to guide their own movements and actions in the game. Although film may act on the body, players act with games, and thus the physical connection with games is distinct and fosters two-way interaction. Moreover, the added haptic involvement, physical interface devices, and extended auditory environment (with the Wii remote speaker, for instance) create additional multimodal interactions between vision, audio, and haptics. ${ }^{123}$

As previously stated, "interactivity" can have different meanings in different contexts, but Collins uses the term specifically to refer to the two-way process between a human user and a system, the experience of which is "embodied because it is inextricably bound to our sensorimotor experience, and our perception is always tied to a mental reenactment of our physical, embodied knowledge."124

\footnotetext{
120 Karen Collins, Game Sound: An Introduction to the History, Theory, and Practice of Video Game Music and Sound Design, (Cambridge: MIT Press, 2008).

121 Winifred Phillips, A Composer's Guide to Game Music, (Cambridge: MIT Press, 2014 ), 127.

122 Chion, Audio-Vision, 24.

123 Collins, Playing with Sound, 22.

124 Ibid., 17.
} 
With open works, musical performers are tasked with becoming active participants in the structural configuration of the performed artifact. Inversely, digital game players are sometimes asked to become musicians. This involves, in part, the utilization of what Chion calls reduced listening, which "takes the sound ... as itself the object to be observed instead of as a vehicle for something else." 125 It can also involve musical authorship, which I define as the creation of novel musical configurations, whether compositionally (prearranged) or improvisationally (in real-time). Since all digital games are dynamic artifacts (the focus of the next chapter) and thus contingent on player action, all games that make sound afford musical authorship to some degree. However, as the number of musical affordances (i.e. the openness the artifact) increases, so too does the potential for substantially unique musical configurations. Furthermore, the aforementioned objectives that usually structure gameplay can be orthogonal or even opposed to more subjective musical goals. We might consider musical authorship an ontological activity - a process of discovering and realizing a possibility space. "To the extent that players are engaged with the goal-oriented, action/reward structure of gaming, they hardly have time to pause and consider ontological problems. The ontological effect is therefore strongest when videogames are less game-like and more open, allowing the mind to wander, experiment, and play." ${ }^{26}$ (Authorship is discussed further in Chapter 4.)

125 Chion, Audio-Vision, 29.

126 Bartee, "Ontological Toys," 59. 


\section{Case Study: Guitar Hero (2005)}

Guitar Hero is one of the most successful and often-cited music game franchises. Most typically, it is described as a rhythm game, which could be more generally classified as a musical accuracy game. Its visual interface involves descending circles that represent musical events within a song, which plays synchronously. (Figure 15) When the circles overlap with indicators at the bottom of the screen, they player is expected hold down the corresponding key on the guitar-shaped game controller and trigger the strum bar. (Figure 16) Successfully playing a note at the right time gains points, increases a point multiplier, and increases the "Rock Meter," which could be considered a form of energy. Playing notes inaccurately or missing them altogether results in the loss of energy and losing too much energy on the Rock Meter causes the stage to end, at which point the player must restart or quit. (This punishment mechanic is discussed further in the "Success-Failure" section below.) The pattern of scrolling musical events generally corresponds to the song's melodic contour or chord fingerings, though a particular button might represent several different pitches over the course of a level. 


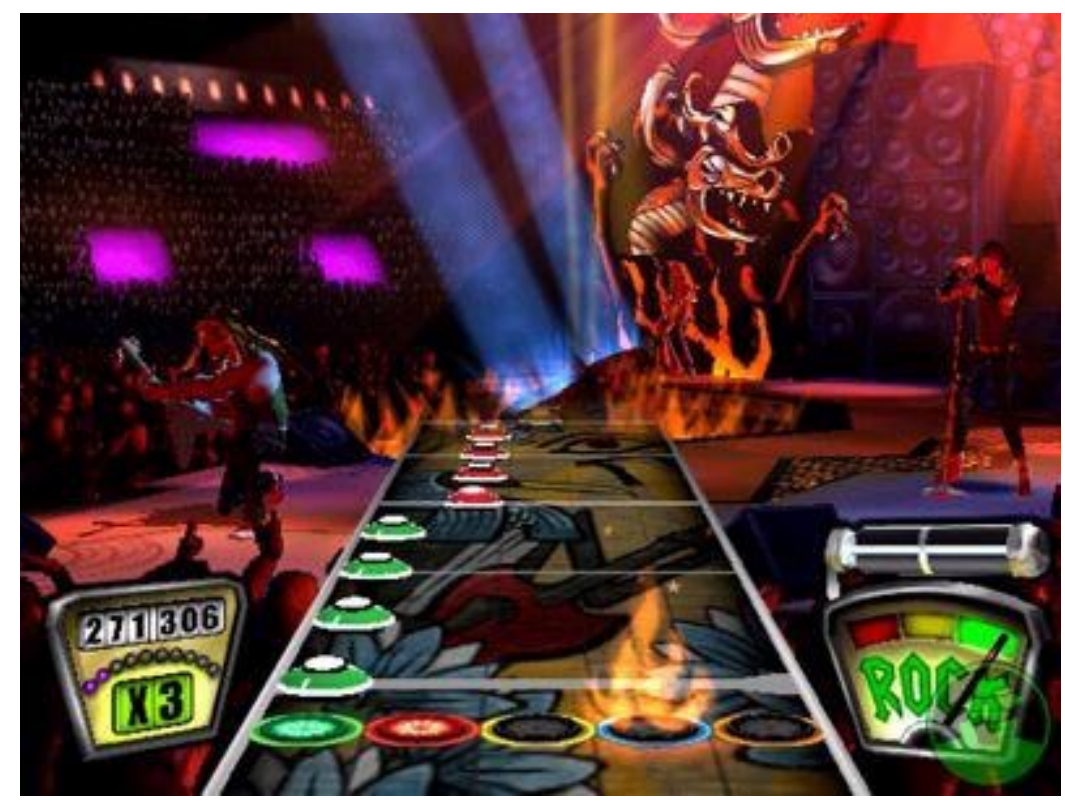

Figure 15. Visual interface of Guitar Hero ${ }^{127}$
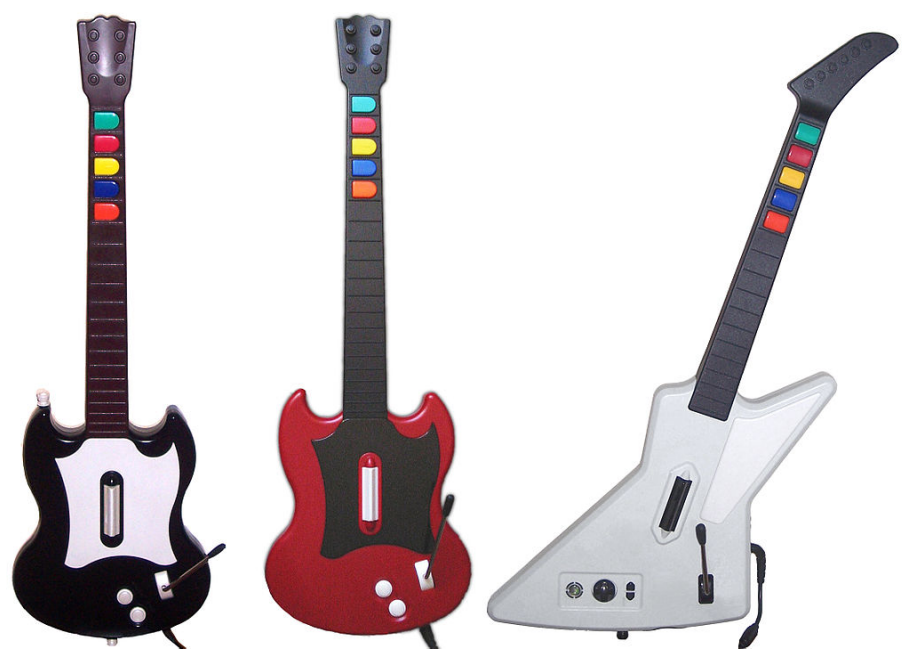

Figure 16. Guitar Hero controllers ${ }^{128}$

127 "Guitar Hero (video game)," Wikipedia, https://en.wikipedia.org/wiki/Guitar_Hero\#/media/File:Guitarhero-screen.jpg [accessed 2016FEB01].

128 Ibid., https://en.wikipedia.org/wiki/Guitar_Hero\#/media/File:Guitar_Hero_series_controllers.jpg [accessed 2016FEB01] 
While Guitar Hero certainly involves musical comprehension, the degree of authorship afforded to the player via the game's design is actually relatively low. The player is allowed to choose which song to play and the difficulty level, yet once a song begins the succession of notes is always the same; the sequence of discrete triggers is either played correctly or it isn't. In this manner, gameplay in Guitar Hero seems closer to the realization of a more traditional linear composition than an open musical work; there is a single prescribed path rather than a class of goals. This is not to say that the experience of playing Guitar Hero precludes creativity more generally. In her book Playing Along, Kiri Miller reveals that some players do express themselves creatively when playing Guitar Hero in the form of physical gestures, custom instruments, and social connections. She also points out that playing Guitar Hero can complement and inform other musical practices by helping players to "listen like musicians" and by affording analytical insights through repeat performances. ${ }^{129}$

\section{Case Study: Electroplankton (2005)}

Released in the same year as Guitar Hero, Electroplankton more closely represents the sandbox category of music games than Guitar Hero. The game was created for the unique capabilities of Nintendo DS (a handheld gaming console), which includes features like a touch-sensitive LCD screen and a microphone. Each of Electroplankton's ten stages utilizes

129 Kiri Miller, Playing Along: Digital Games, YouTube, and Virtual Performance, digital edition, (New York: Oxford UP, 2012), 124. 
the device's input mechanisms to make sound in a different way, effectively providing ten different types of musical composition-instruments.

In an article about Nintendo's unique style of incorporating musical play into their games, Roger Moseley and Aya Saiki explain that Electroplankton's designer, media artist Toshio Iwai, was inspired by the flip book and music box, and regards digital game systems as "musical instruments with which one can play with moving images and music simultaneously." 130 As a young man, Iwai played video games like Super Mario Bros. and Xevious as musical instruments - jumping or shooting, respectively, along with the background music — which satisfied his desire for childlike play (i.e. paidia). ${ }^{131}$ Effecting this sort of play in a system full of rules and constraints and using relatively simple devices to create complex phenomena points toward an artistic aesthetic that Moseley and Saiki argue is typical in Japanese culture and can be found in many other aspects of Japanese life, such as Edo-era woodblock prints and haiku. ${ }^{132}$ It also reinforces the notion the games are fundamentally toys that can be experienced in spite of prescribed rules.

In one stage, four electroplankton of the type Luminaria move around the screen. Each moves at a different rate and creates sound in a particular pitch range upon collision with nodes. (Figure 17. Note that images only show the active touch-sensitive screen of the DS.) Players can touch any of the thirty-six nodes to change its rotation, which alters the

130 Toshio lwai, Iwai Toshio no shigoto to shuhen (Tokyo: Rikuyosha, 2000), 64.

131 Roger Moseley and Aya Saiki, "Nintendo's Art of Musical Play," Music in Video Games: Studying Play, Eds. K.J. Donnelly, William Gibbons, and Neil Lerner, (New York: Routledge, 2014), 58-9. 132 Ibid., 53. 
path of any Luminaria that encounters it and thus changes the overall sonic result. Through a very simple touch interface, players make decisions about pitch, tempo, melodic length, harmonic density, and overall duration.

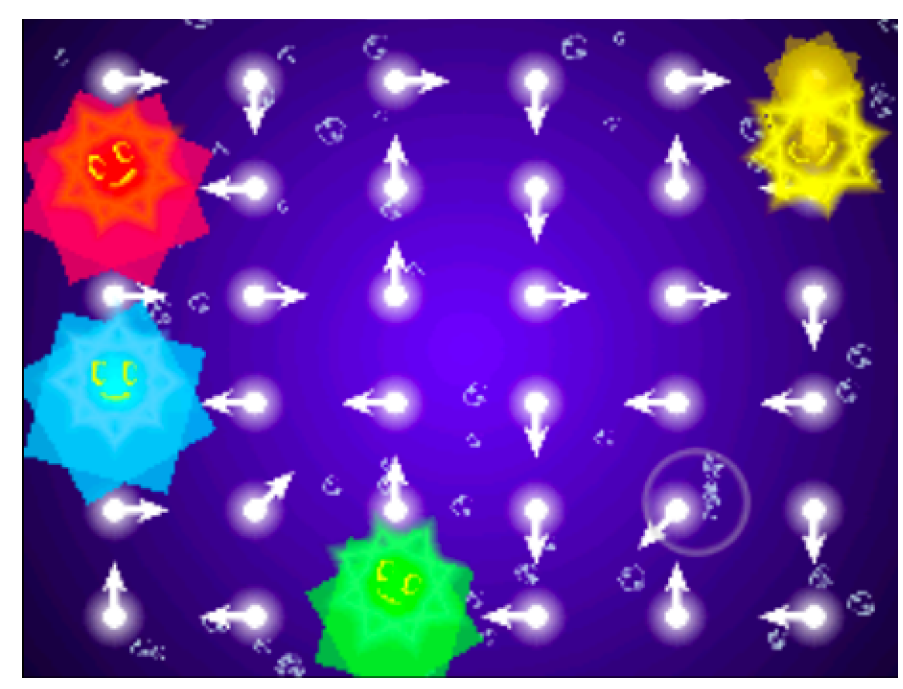

Figure 17. Electroplankton, Luminaria stage

In another stage, five electroplankton named Lumiloop are controlled via the stylus and touchscreen. Players touch the tip of the stylus anywhere within the torus shaped agent and drag the stylus in a circular motion causing the Lumiloop to rotate. Each Lumiloop generates a particular ambient tone that is proportional to the speed at which it rotates, and the speed of the Lumiloop's rotation, as well as the size of its aura, correlates to how fast the player's stylus moves. In this stage, the sense of musical creation is even more embodied than the Luminaria stage since the amount of physical energy expended is mapped directly to the intensity of the sound (kinesonic synchresis). 


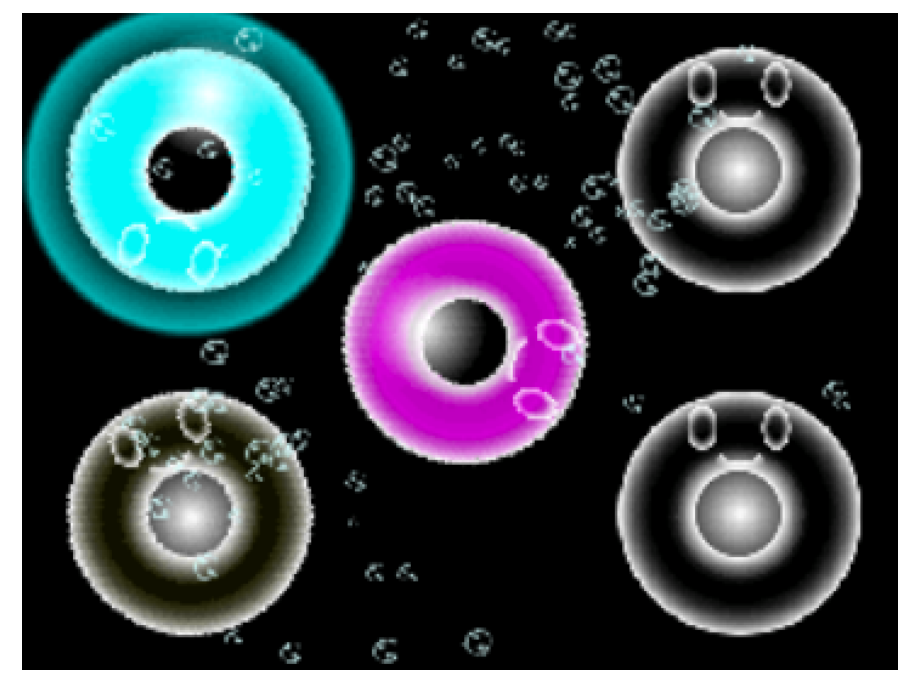

Figure 18. Electroplankton, Lumiloop stage

In yet another stage, players are asked to create sounds themselves, which are picked up by the microphone and recorded into game memory. The sounds are sucked up by the Volvoice electroplankton, who then repeats the sound with different timbral qualities depending on its user-defined shape.

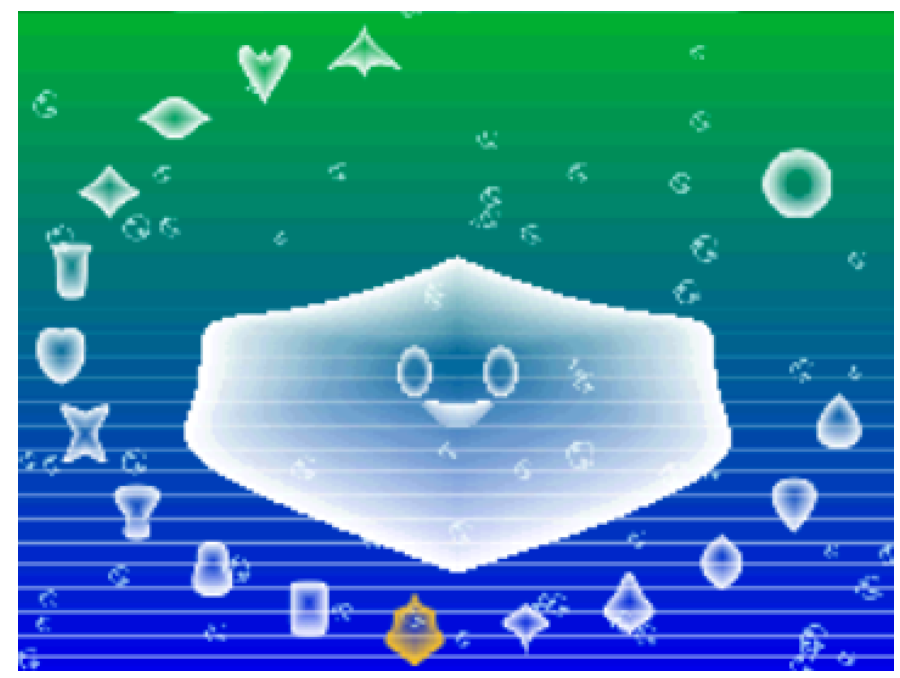

Figure 19. Electroplankton, Volvoice stage 
Electroplankton affords musical authorship to a greater extent than Guitar Hero; by way of its design, the player has relatively more freedom to create novel musical configurations within the parameters of the game. Again, this does not mean that one game necessarily involves more player creativity than the other, nor does it mean that one is more performed. As Steven Reale states, "games keep score by tabulating and evaluating a player's ability to accomplish its tasks, but when game scores and musical scores collide, we might consider player performance literally: that the playthrough becomes - more than a measure of skill - a kind of expression to be judged aesthetically."133 Both Guitar Hero and Electroplankton are performed and involve aesthetic expression, but Electroplankton's designed affordances make it better suited for musical authorship.

As previously stated, the distinction between accuracy games like Guitar Hero and sandbox games like Electroplankton is not discretely defined. Increasingly, game designers have attempted to blur such a distinction. With accuracy-based musical games, more musical authorship is afforded by expanding the possibility space. For example, in the musical accuracy game Rock Band 4 (2015), a soloing system was introduced to the franchise that allows players to improvise musical phrases within the constraints of the system. On the other hand, musical sandbox games like Electroplankton achieve a more game-like quality by imposing more constraints and limiting the possibility space. Furthermore, the style

133 Steven Beverburg Reale, "Transcribing Musical Worlds; or, Is L.A. Noire a Music Game?” Music in Video Games, 102. 
and playfulness of the interface can greatly affect how gamers perceive and thus interact with the system.

\section{Musicians as Gamers}

According to Hunicke, LeBlanc, and Zubek, considering the player's ultimate emotional responses first when creating a game facilitates an experience-driven design process. This is important because "games are more like artifacts than media. By this we mean that the content of a game is its behavior - not the media that streams out of it towards the player."134 To better describe and understand the player's experience, they suggest some common aesthetic goals:

Sensation: Game as sense-pleasure

Fantasy: Game as make-believe

Narrative: Game as drama

Challenge: Game as obstacle course

Fellowship: Game as social framework

Discovery: Game as uncharted territory

Expression: Game as self-discovery

Submission: Game as pastime

If we apply this taxonomy to a musical context, many (if not all) of these goals retain their relevance. Sensation, challenge, and fellowship seem to already be intrinsically part of any public musical performance, albeit to various degrees. Perhaps one of the least applicable goals in a musical context would be narrative in a literal sense, though some musical scholars use the concept of narrative in a more metaphorical sense. For example,

134 Hunicke, et al., "MDA". 
Agawu includes narrative as one of his six criteria for analyzing musical meaning, and uses the term frequently and broadly to describe the development of various musical concepts over the course of a piece. In his words, "The idea that music has the capacity to narrate or to embody a narrative, or that we can impose a narrative account on the collective events of a musical composition, speaks not only to an intrinsic aspect of temporal structuring but to a basic human need to understand succession coherently. Verbal and musical compositions invite interpretation of any demarcated temporal succession as automatically endowed with narrative potential."135 The aesthetic goal of fantasy is perhaps questionable in a musical context, though it could be argued that musical performances often serve as a way of transcending the mundane, as Small suggests in his assessment of musicking and its direct relationship to ritual.

The remaining goals are particularly significant with respect to open performed artifacts. With any piece of music, expression ("self-discovery" or self-articulation) is a critical goal, at least implicitly, yet it is explicitly central to open works in which players are expected to take part in musical authorship. Similarly, discovery ("uncharted territory") is important when access to information is controlled or limited and when surprise is a deliberate factor, as is often the case with interactive performed artifacts. (More on this in Chapter 3.) Ostensibly, all musical performances, like games, are voluntary, and therefore include the goal of submission ("pastime"). However, I would argue that as play ("manipulation

135 Agawu, Music as Discourse, 102. 
that indulges curiosity" 136 ) increases in a performance, so too does the sense of submission. Eno describes submission as being the opposite of control (yet another continuum):

What we're not so used to is the idea that another great gift we have is the talent to surrender and to cooperate. Cooperation and surrender are actually parts of the same skill. To be able to surrender is to be able to know when to stop trying to control. And to know when to go with things, to be taken along by them. And that's a skill that we actually have to start relearning. Our hubris about our success in terms of being controllers has made us overlook that side of our abilities. So we're so used to dignifying controllers that we forget to dignify surrenderers. ${ }^{137}$

He relates surrender to religion, sex, art, and drugs, which all tend to be "either totally dignified or totally taboo" in the vast majority of our world's cultures. ${ }^{138}$

\section{Success-Failure}

Asking a classically trained musical performer to literally play during a performance can be problematic, despite the fact that "play" is the de facto descriptor for such an activity. Traditionally, performers undergo rigorous training within a paradigm that does not tolerate failure. As such, it is understandable how being put into a situation where failure is likely might cause performer anxiety. Beyond aesthetic goals, digital games often have very specific and idiosyncratic objectives that musical scores (usually) do not. Such objectives may include successful navigation through perilous terrain, the destruction of enemy forces, pattern matching, etc. More generally, game players usually aim to

\footnotetext{
136 Schell, Art of Game Design, 30.

137 Eno, "Composers as Gardeners."

138 Ibid.
} 
complete a stage, earn an achievement, beat a friend, get the high score, etc. In other words, they strive for elements of designed endogenous [in-game] value, which serve to create compelling conflict. ${ }^{139}$ Implicit in this objective-based paradigm is the ability of the game to quantitatively measure success or failure and to react accordingly.

In an article titled, "Fear of Failing? The Many Meanings of Difficulty in Video Games," Jesper Juul claims that "failure adds content by making the player see new nuances in the game."140 He defines a common progression of player punishment, beginning with 1) energy punishment, which brings the player closer to 2) life punishment (loss of a "life" unit), which brings the player closer to 3) game termination punishment. Additionally, failure may result in 4) setback punishment, which means losing territory or abilities. He adds that "all failures eventually translate into setbacks, and the player's use of time and energy is the most fundamental currency of games." 141 Juul conducts a study in which he finds that players simultaneously do not want to fail yet do not want to win without failing. To explain this phenomenon, he invokes Mihaly Gsikszentmihalyi's often-cited concept of flow. Simply stated, players of any game become bored if there is not enough challenge, yet become discouraged if there is too much challenge. As such, the thresholds of flow in a game are determined by a player's skill level and the game's difficulty level. (Figure 20) When the optimal blend of challenge to skill is achieved, the player has reached the state of flow. In the state of flow, a person experiences a merging of action and awareness, a

\footnotetext{
139 Schell, Art of Game Design, 32.

140 Jesper Juul, "Fear of Failing? The Many Meanings of Difficulty in Video Games," Eds. Mark J. P. Wolf \& Bernard Perron, The Video Game Theory Reader 2, (New York: Routledge 2009), 237.

141 Ibid., 238.
} 
centering of attention, loss of ego, and better control of action and environment.

Furthermore, the state of flow is autotelic; it appears to need no goals or rewards external to itself. ${ }^{142}$ Phillips relates flow to a sense of immersion that "takes place when the gamer loses consciousness of the methods of perception and interaction with the game."143

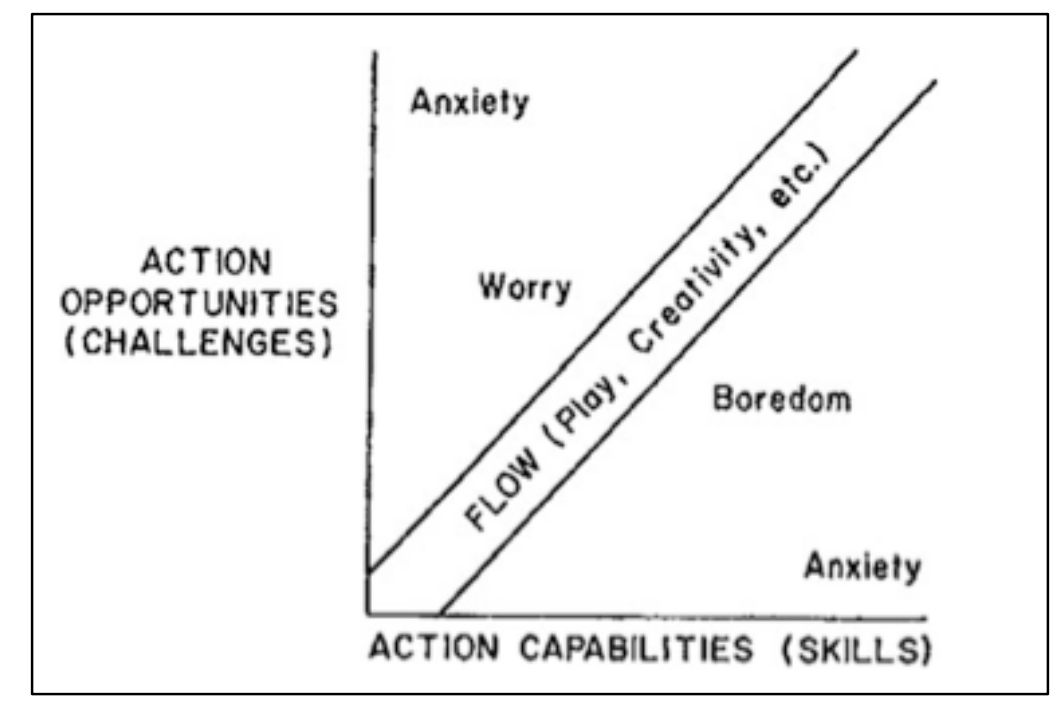

Figure 20. Csikszentmihalyi's model of flow ${ }^{144}$

Game designer Jenova Chen draws from Csikszentmihalyi's idea of flow in suggesting the benefit of dynamic difficulty adjustment (DDA) in games. He explains that, "In order to realize optimal experiences for a much wider audience, not only do we need to offer a wide Flow Zone coverage, we also need a highly adaptive system to weave the rich

\footnotetext{
142 Mihaly Csikszentmihalyi, Flow and the Foundations of Positive Psychology: The Collected Works of Mihaly Csikszentmihalyi, (Springer: New York, 2014), 145.

143 Phillips, A Composer's Guide to Game Music, 37.

144 Csikszentmihalyi, Flow, 147.
} 
gameplay experiences together, adjusting Flow experiences based on the players." 145

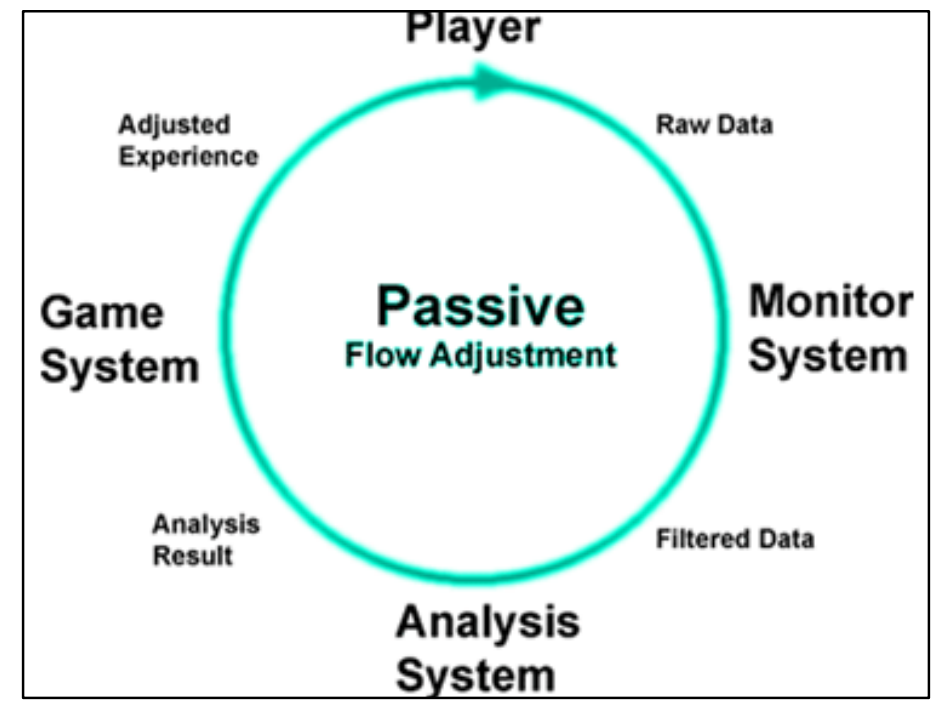

Figure 21. Chen's model of dynamic difficulty adjustment (DDA)

Zorn stresses the importance of balancing challenge and fun in creating his game scores, and finds that inviting improvisation into a composition is highly conducive to achieving that balance since openness can accommodate different skill levels. He also states: "I wanted to find something to harness the personal languages that the improvisers had developed on their own, languages that were so idiosyncratic as to be almost unnotatable (to write it down would be to ruin it). The answer for me was to deal with form, not with content, with relationships, not with sound." 146 Just like in digital games, the demands of the score and the skill of the performer also contribute to a flow zone, and with dynamic

\footnotetext{
145 Jenova Chen, "Flow in Games," (master's thesis, University of Southern California, 2006), http://www.jenovachen.com/flowingames/designfig.htm [accessed 2016FEB06].

146 Zorn, "The Game Pieces," Audio Culture, 199.
} 
game-based performed artifacts that zone has more flexibility.

\section{Case Study: Paul Turowski, GENIV (2013)}

$G E N I V$ was created for the Dither electric guitar quartet, an ensemble well-known for their prowess with openness, improvisation, and game scores. (Their first album, Dither Plays Zorn, features realizations of John Zorn's game pieces.) GENIV involves four discrete channels of audio input that are each processed and regulated separately in a Max patch. The musical score for the piece is presented to players on iPads, which are all connected via a local area network.

In Stage 1, players swipe on the screen to initiate movement of the flashing column in the center known as the "paddle." (Figure 22) After the paddle is activated, players quickly strum the indicated notes using any articulations or effects they feel are appropriate. The amplitude of their strum is represented by a small circle (“amp meter") moving from left (piano) to right (forte). Players match the amp meter to the paddle to gain more energy

(i.e. a higher score) and the order of accuracy determines the given pitches to play (based on precomposed pitch sets). (Figure 23) Furthermore, energy and paddle velocity determine the outcome of several aspects of future stages, such as the tonality of the next section. A player's current energy level is indicated by the meter bar on the left side of the screen. Since loudness is mapped to dynamic score elements, it is governed by the affordances, constraints, and feedback of the designed system (ecology). Meanwhile, the 
specification of pitches, which determines composed harmonic relationships, is rule-based (economical); it is not reinforced by the affordances and constraints of the system and can be easily ignored.

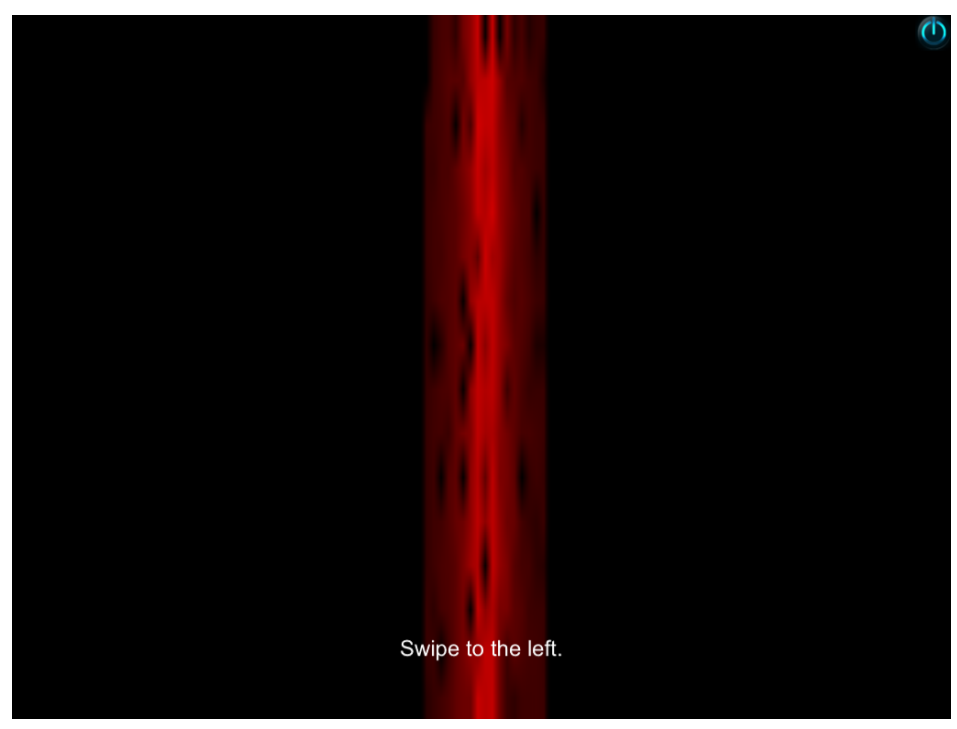

Figure 22. Turowski, GENIV, Stage 1. Initialization

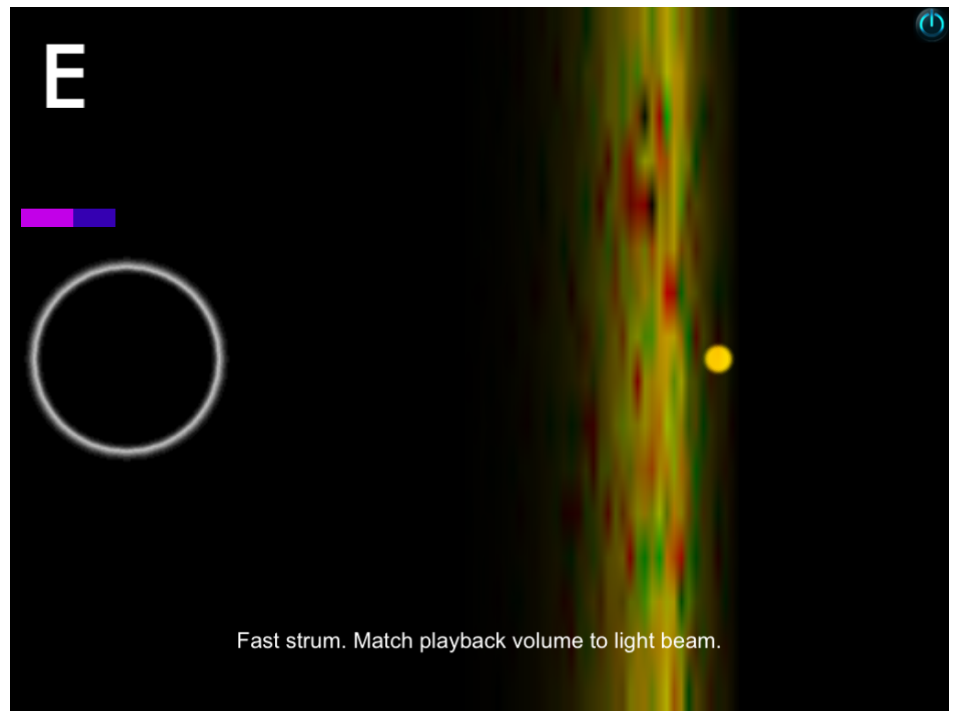

Figure 23. Turowski, GENIV, Stage 1. Volume control 
In Stage 2, players may touch the screen to select one of the available "spawners." (Figure 24) Once a spawner is chosen (either by the player or at random if no input in given), it will indicate that the audio input of the performer is being recorded by showing a microphone icon. As in the first stage, players are instructed to play within the pitch set displayed on the left side of the screen. Objects spawned by the spawner represent recorded buffers of audio that are played back electronically. (Figure 25) Throughout each spawner's life span, the visual pattern of sound objects is saved into memory and displayed in miniature on the right side of the screen. (Figure 26) After a spawner expires, the process is repeated until all spawners have expired.

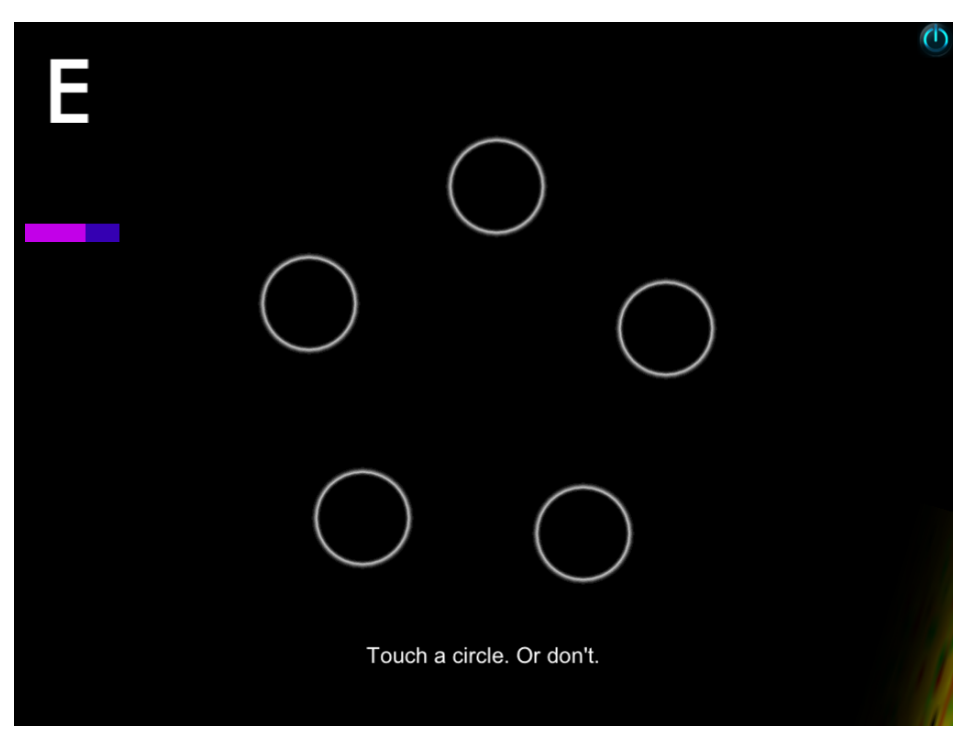

Figure 24. Turowski, GENIV, Stage 2. Spawner selection 


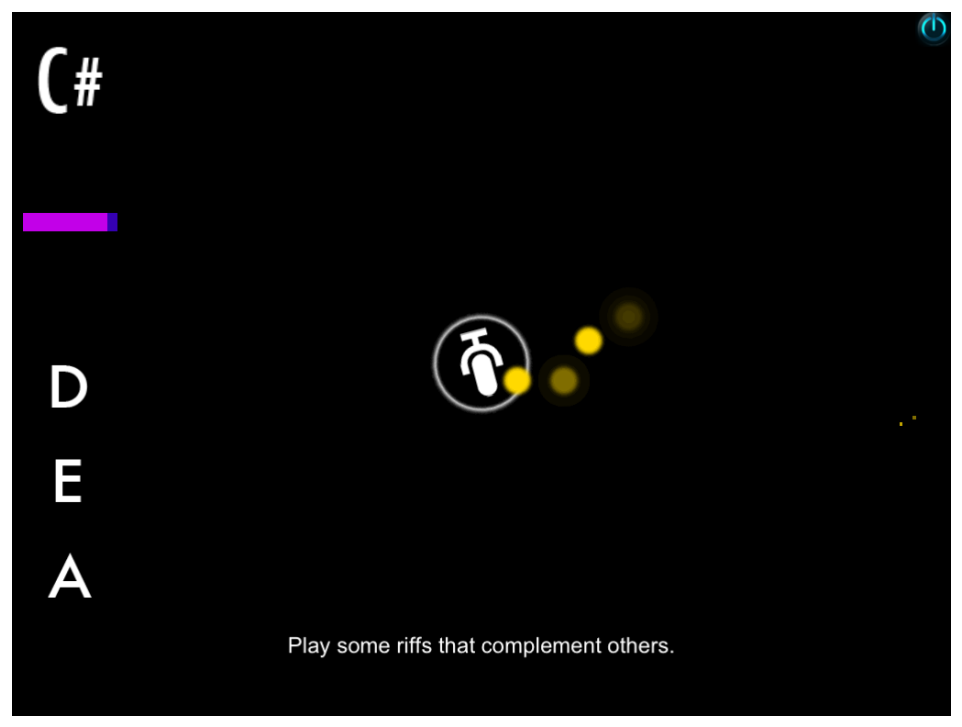

Figure 25. Turowski, GENIV, Stage 2. Spawned instances

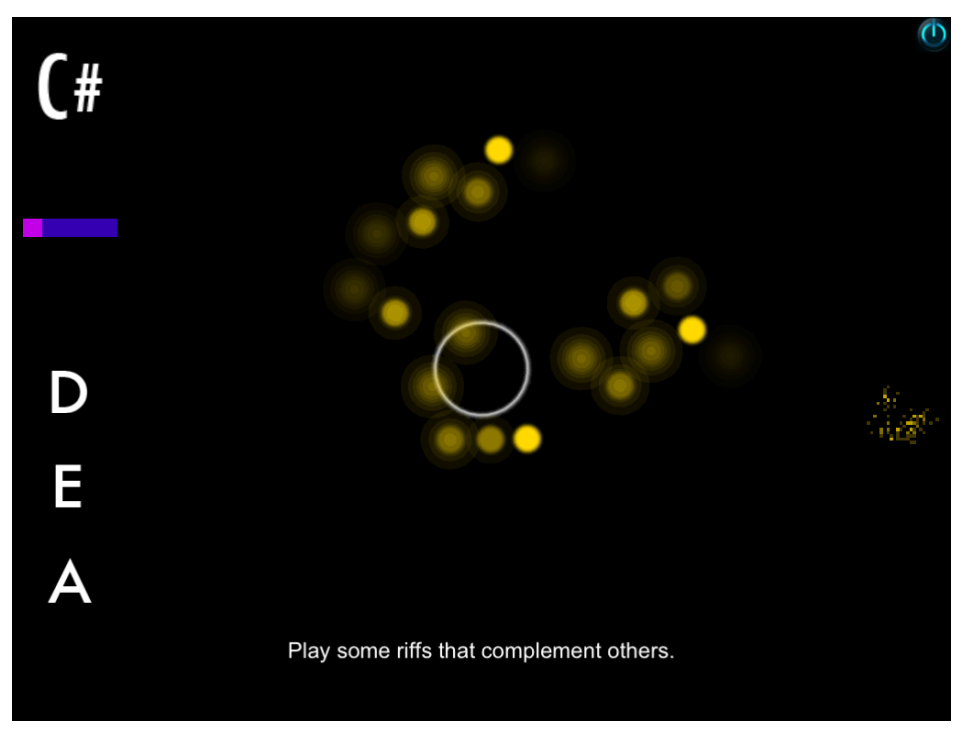

Figure 26. Turowski, GENIV, Stage 2. Emergence of the artifact

In Stage 3, patterns created in Stage 2 are recalled in an order that relates to player rankings (i.e. Stage 1 accuracy). The player may touch the parts of the screen in which these patterns appear while performing hammer-ons/pull-offs with the indicated notes. 
Touching a bright part of a pattern generates more energy allowing more sound (both direct and previously recorded) to be heard. The current energy level is displayed using the meter bar on the left. (Figure 27) If there is no pattern displayed, the performer cannot play audibly since the software silences their incoming audio signal. Once again, constraints reinforce the rules of the piece.

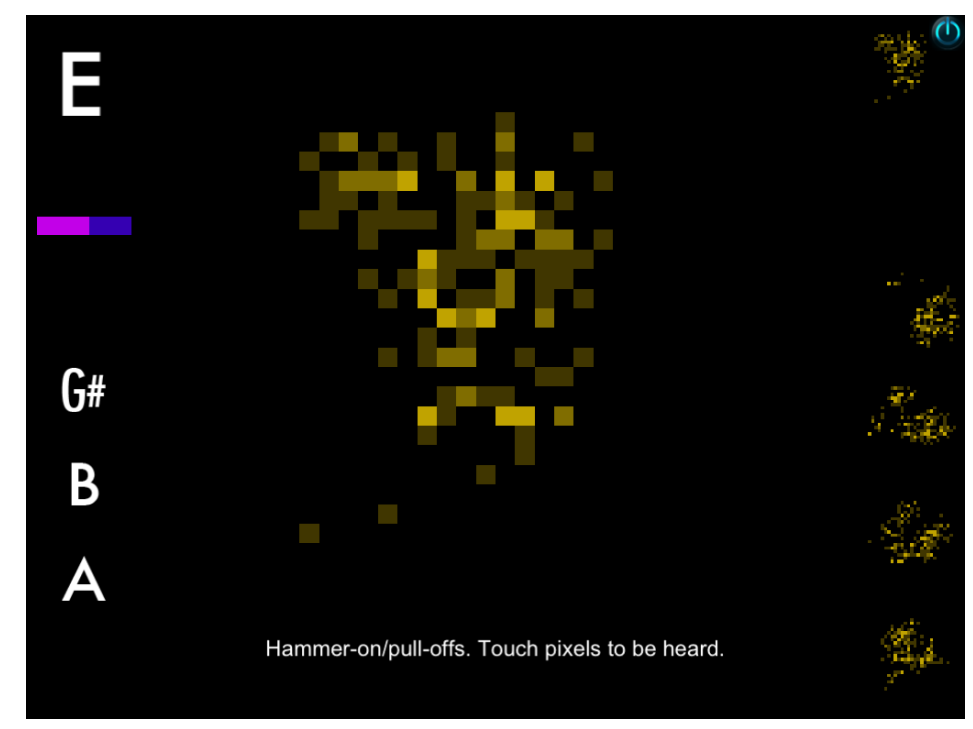

Figure 27. Turowski, GENIV, Stage 3. Playing the artifact

Once the players reach Stage 4, the textures from the previous stage become particles in a cloud. Cloud position and brightness correspond to patterns drawn by the player in Stage 3. Players are asked to play freely within the indicated pitch set on the left side of the screen and with a dynamic level equal to the cloud's brightness. (Figure 28) Rankings from Stage 1 are used to determine the order of player drop-outs in this final stage such that the "winner" (i.e. the player with the most accurate amplitude matching) enjoys a solo at the end of the piece. 


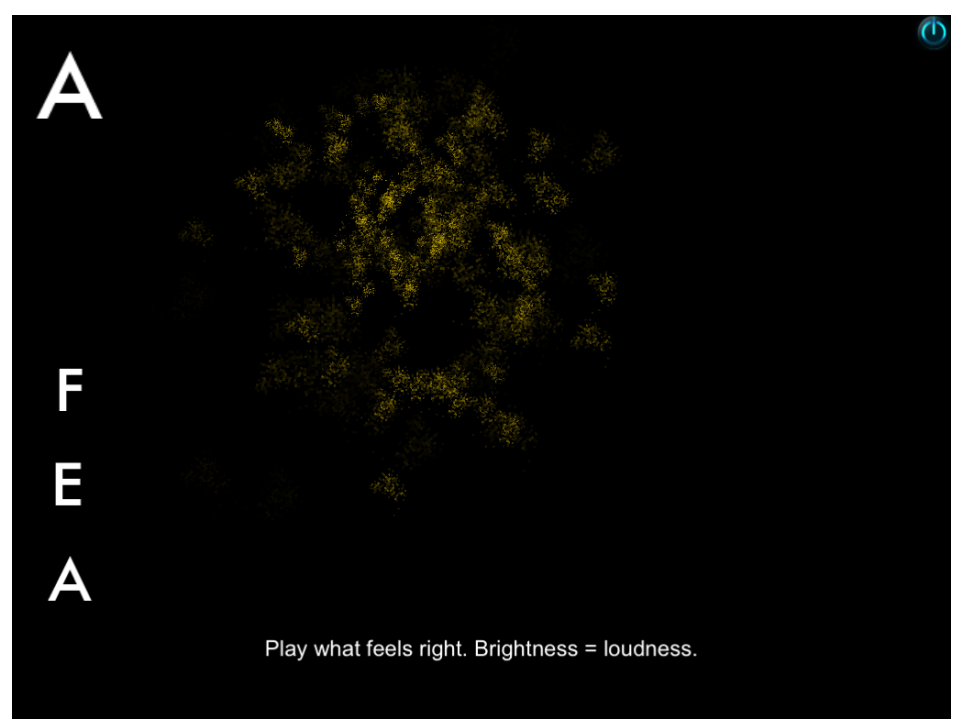

Figure 28. Turowski, GENIV, Stage 4. Improvising with generated clouds

GENIV was inspired by many of the same ideas as Study $\mathcal{N}_{0} .2 . x$, though GENIV is closer to the realm of the digital game. While both rely on animated digital scores, the configuration of the score for GENIV is contingent on player input. Both works could be described as open, but GENIV utilizes affordances, constraints, and specific objectives to reinforce the rules of the piece. 


\section{Chapter 3: Dynamic Artifacts}

\section{Ergodicity and Gybernetics}

In his book, Cybertext: Perspectives on Ergodic Literature, Espen Aarseth describes the mechanism of texts as machines "for the production of variety of expression." $147 \mathrm{He}$ repurposes the term "ergodic"148 to describe a machine in which work is exerted by the receiver (e.g. reader, performer) to physically construct the artifact.

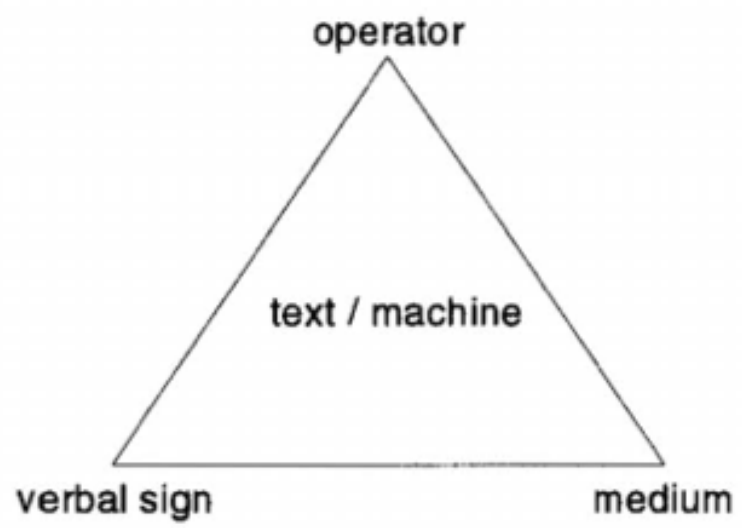

Figure 29. Aarseth's diagram of the textual machine

Aarseth asserts that ergodicity is not defined by the medium itself but rather by the way in which it functions. For example, the ancient Chinese I-Ching could be considered an ergodic text in that it presents the reader with a possibility space which requires non-

147 Espen Aarseth, Cybertext: Perspectives on Ergodic Literature, (Baltimore, London: Johns Hopkins UP, 1997$), 3$.

148 The term "ergodic" is borrowed from physics, and is derived from the Greek works ergon and hodos, meaning "work" and "path", respectively. Ibid., 1. 
trivial extranoematic (i.e. outside the mind) effort to navigate. Aarseth also links the idea of ergodic literature to Penelope Reed Doob’s discussion of labyrinths, which can be described as either unicursal (only one path of traversal) or multicursal (multiple paths) systems. ${ }^{149}$ A key distinction between ergodic texts and the interpretive ambiguities of linear text is that with ergodic texts "you are constantly reminded of inaccessible strategies and paths not taken, voices not heard." 150

The concept of cybernetics has been applied to various contexts, from designing mechanical systems to describing phenomena like natural selection and social behavior. In general, it denotes a system that governs itself via a feedback loop. For example, a steam engine includes a governor, which regulates the fuel intake of the system and thus the movement of the cylinder.

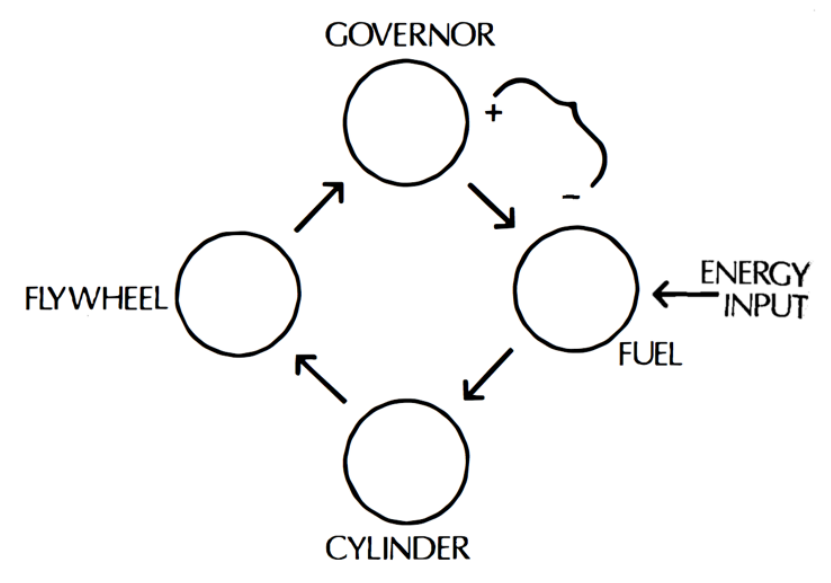

Figure 30. Diagram of a steam engine ${ }^{151}$

149 Penelope Reed Doob, The Idea of the Labyrinth from Classical Antiquity through the Middle Ages, Ithaca: Cornell UP, 1990.

150 Aarseth, Cybertext, 3.

151 Gregory Bateson, Mind and Nature: A Necessary Unity, (E.P. Dutton: New York, 1979), 104. 
Aarseth uses the term cybernetic somewhat loosely to refer both to cybernetic sign production (a model of communication) as well as the mechanics of the media itself. Since the former usage is complex and well covered in other texts ${ }^{152}$, and for the sake of clarity, I subscribe to the latter usage so that cybernetics and ergodicity may be mutually exclusive. For example, the I-Ching is ergodic but not cybernetic, whereas a conventional novel on a digital device is cybernetic but not ergodic. In other words, cybernetic shall henceforth denote a medium's designed self-regulating mechanical quality while ergodic will denote the quality of the traversal functions that are afforded to the user. By this definition, digital games are always cybernetic.

Aarseth broadly defines "text" as "any object with the primary function to relay verbal information," adding that "(1) a text cannot operate independently of some material medium, and this influences its behavior, and (2) a text is not equal to the information it transmits. Information is here understood as a string of signs, which may (but does not have to) make sense to a given observer."153 In his subsequent analyses of various texts, he includes the genre of computer games known as adventure games, in which a player typically navigates through dungeons vanquishing monsters and collecting items and treasure. In such games, elements are represented by graphic images that signify their

\footnotetext{
${ }^{152}$ For a deeper discussion on the various applications of cybernetic principles, including in the arts, see: N. Katherine Hayles, How We Became Posthuman: Virtual Bodies in Cybernetics, Literature, and Informatics, (Chicago UP, 1999).

153 Aarseth, Cybertext, 62.
} 
type and function, which can change over time. Aarseth explains that, "images, especially moving images, are more powerful representations of spatial relations than texts, and therefore this migration from text to graphics is natural and inevitable." $154 \mathrm{He}$ also distinguishes cybertext from narrative in such a context thusly:

"The cybertext reader is a player, a gambler; the cybertext is a gameworld or world-game; it is possible to explore, get lost, and discover secret paths in these texts, not metaphorically, but through the topological structures of the textual machinery. This is not a difference between games and literature but rather between games and narratives. To claim that there is no difference between games and narratives is to ignore essential qualities of both categories. And yet, as this study tries to show, the difference is not clear-cut, and there is significant overlap between the two."155

This definition of text and the conclusions that Aarseth draws can be easily extended to musical works, particularly open works. All musical notation conveys verbal information that could be rewritten using words, yet graphic images tend to be favored because they are better at relating spatial (i.e. temporal) relationships. Of course, musical scores (especially those that use non-traditional notation, such as graphic scores) often include text that explains how graphic symbols should be interpreted, especially if those symbols are non-standard. Open musical scores are closer to cybertext than they are to narrative, and cybertexts are closer to open works than unicursal scores. The performance of any musical work requires non-trivial effort, but the performer of the open musical score actively configures the scriptons of the performed artifact while the performer of a more

\footnotetext{
154 Aarseth, Cybertext, 102.
}

155 Ibid., 4-5. 
traditional fixed and linear score does not.

\section{Ergodic Classification}

Aarseth proposes a typology that regards units of textual information as either textons or scriptons - strings of signs as they exist in the text or as they exist to the reader, respectively. For example, in hypertext (e.g. an interconnected group of web pages), a textual passage may be invisible to the reader until a particular link is clicked. Once the textons in this passage are revealed they become scriptons, and their meaning may be influenced by their adjacency to other scriptons. If the passage is never revealed, its strings of signs remain only textons, which then make up, along with other unrealized textons, the "inaccessible strategies and paths not taken, voices not heard." Aarseth's typology is comprised of several attributes that relate to how textons and scriptons function: 1) dynamics (static, intratextonic [texton quantity conserved], or textonic [texton and scripton quantity variable]), 2) determinability (scripton adjacency), 3) transiency (affect of passing time) 4) perspective (user identification with a character), 5) access (texton availability), 6) linking (link access), and 7) user functions, which define the

role of the user in relation to the artifact. The interpretive function exists in all texts and is thus not a distinguishing quality of ergodic works. The explorative function is exemplified by the hypertext, which allows the user to choose a path of traversal through the text even though scriptons remain mostly preconfigured. Cybertexts allow for the configurative function (scriptons are configured by the user) and the textonic function 
(textons or traversal functions can be added to the text).

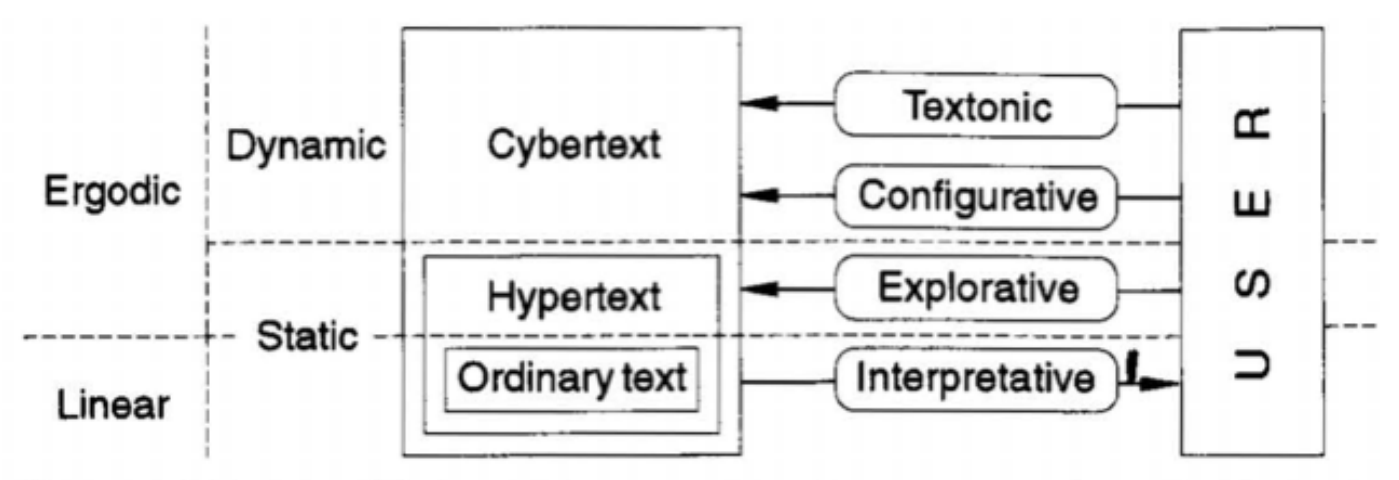

Figure 31. Aarseth's diagram of user functions ${ }^{156}$

Returning to the music game comparison in the previous chapter, we can better understand the distinction between Guitar Hero and Electroplankton through this ergodic typology. In the latter, determinability (scripton adjacency) is more variable, allowing for more possible combinations of musical information and thus a greater capacity for player authorship. By extension, the user function is also slightly different; both are configurative to some degree, but Electroplankton is both more configurative and also textonic, since players may add their own sounds in the Volvoice stage. (See Chapter 2.) In other words, ergodicity and potential player authorship are closely correlated.

\section{Meta-Composition}

Another lens through which to look at complex performed artifacts is the concept of the

156 Aarseth, Cybertext, 64. 
meta-composition - i.e. a composition that can create other compositions - as defined in the doctoral dissertation of performer and composer Curtis Bahn. ${ }^{157}$ The meta-composition is inspired by Pamela McCorduck's discussion of meta-art ${ }^{158}$ and Stephen Holtzman's notion of the metamedium ${ }^{159}$, which is conceptualized as intelligence amplification (I.A.) rather than artificial intelligence (A.I.); the machine augments the consciousness of the human that programmed it rather than serving as a completely autonomous consciousness. This philosophy seems to be more consonant with Meillassoux's correlationism, which privileges human-world relationships, than OOO's flat ontology. (See Chapter 1.) In language very similar to Aarseth's, Bahn describes the qualities of a system that produces meta-compositions: "Given a machine for producing text, there can be three main positions of human-machine collaboration: (1) preprocessing, in which the machine is programmed, configured, and loaded by the human; (2) coprocessing, in which the machine and the human produce text in tandem; and (3) postprocessing, in which the human selects some of the machine's effusions and excludes others. These positions often operate together: either 1 and 2; 1 and 3; or 1, 2, and 3; or 1 by itself, although the human operator need not be the same in different positions."160 He also explicitly relates the meta-composition to improvisation and postwar experiments with indeterminacy, like the open works of Wolff, et al.

\footnotetext{
157 Curtis Robert Bahn, "Composition, improvisation and meta-composition," (Ph.D. diss., Princeton University, 1998). In ProQuest Dissertations and Theses, http://search.proquest.com/docview/304373240?accountid=14678 [accessed November 10, 2014$].$

158 Pamela McCorduck, Aaron's Code, Meta-art, Artificial Intelligence, and the work of Harold Cohen, (New York: W.H. Freeman and Company, 1991). 159 Stephen Holtzman, Digital Mantras, (Cambridge: MIT Press, 1996).

160 Aarseth, Cybertext, 135.
} 
Case Study: Christian Wolff, For 1, 2 or 3 People (1964)

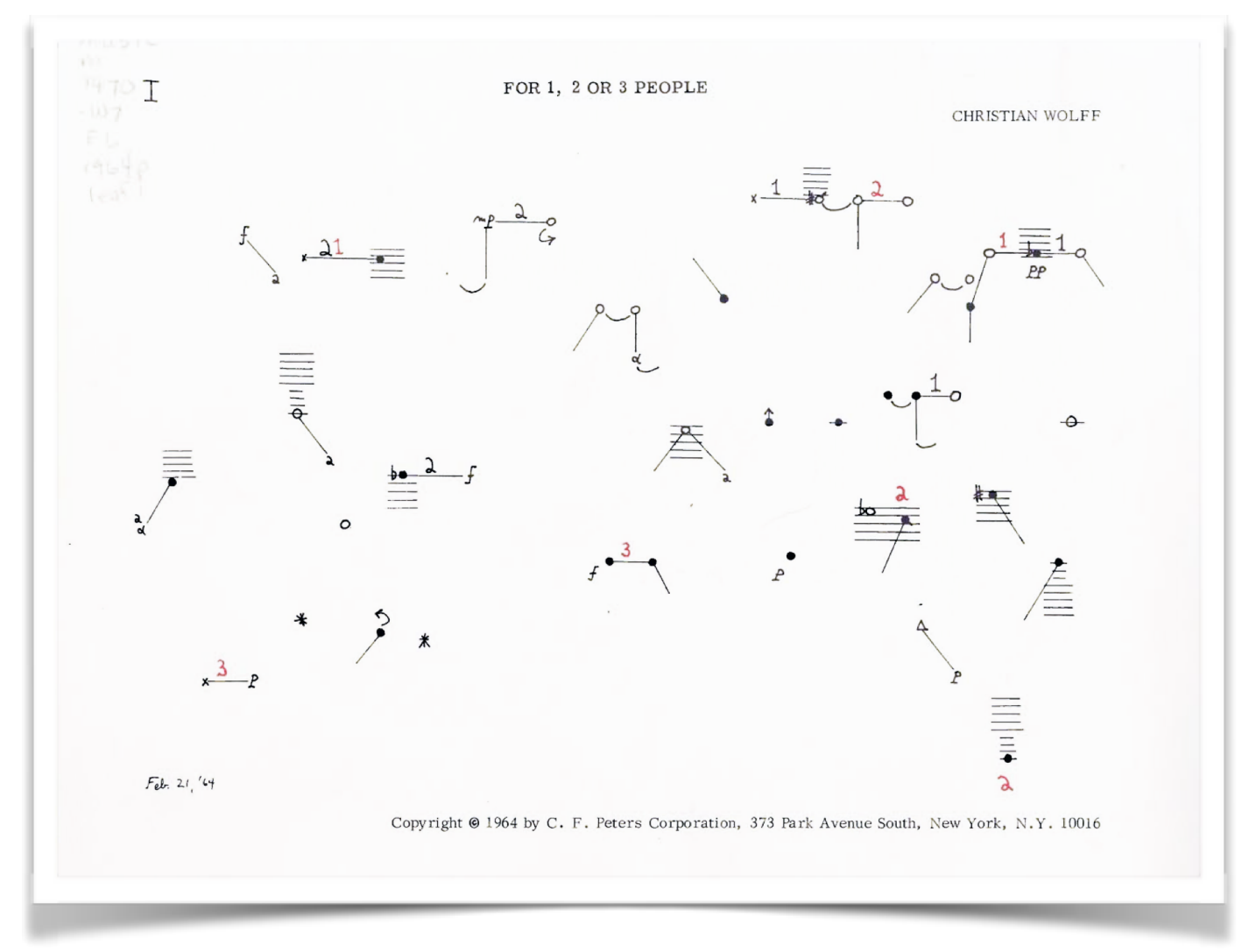

Figure 32. Wolff, For 1, 2 or 3 People, pg. 1 of 10

In For 1, 2 or 3 People, musical events (i.e. textons) are distributed on each of ten pages.

For the purpose of this analysis, an event is defined as a spatially isolated sound or group of sounds. A performance can be comprised of any number of pages to be played without repetition, or any one page repeated no more than ten times. Each performer must play everything on a page in any sequence, except for the ninth page where any number of events can be played or omitted any number of times. Minimally, a performer could 
choose to play just one event from the ninth page one time. Maximally, a performer could choose to play all pages and repeat the ninth page ad infinitum. Thus, the duration of the piece can be anywhere from the length of one musical event to as long as any performer wishes to (or can) play.

One might expect an even spatial distribution of events; a linear layout might look too much like a traditional score and be read in the same manner between performances and performers. (Even distributions are used in similarly notated works such as Feldman's Intermission 6 or Stockhausen's Klavierstïck XI.) However, Wolff widely varies the distribution from page to page. (Figure 33) Some pages are fairly even and non-linear (page III) while others are clearly more linear (page VII, which also includes unexplained markings of "1." and "2." before the second and third row from the top, respectively).

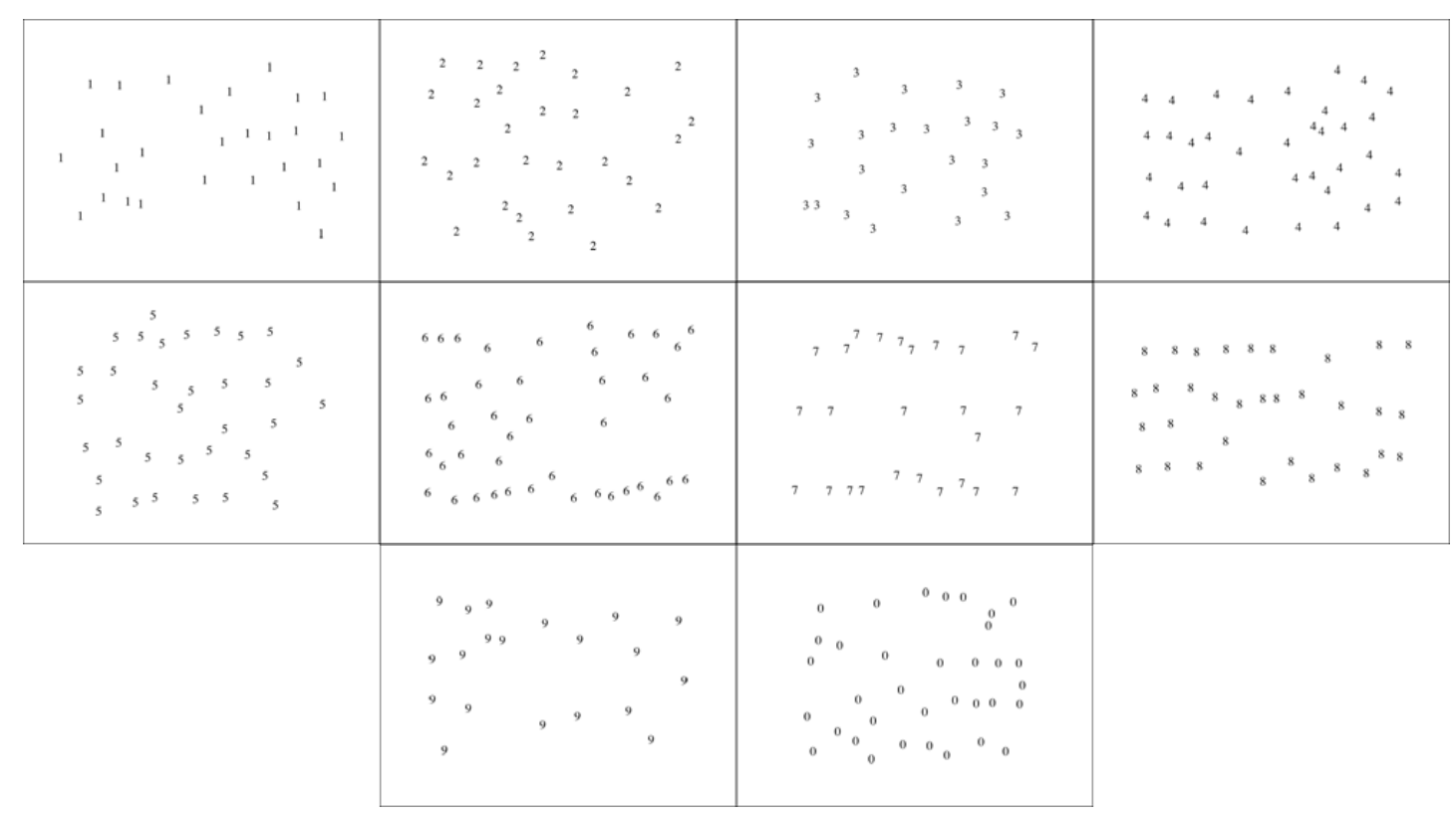

Figure 33. Wolff, For 1, 2 or 3 People. Spatial distribution of events 
Each page features unique syntactic formations which, as Thomas DeLio explains, "yield a different complex of interactions"; e.g. page III features multiple seven-second rests, whereas page IV features many two-second rests. ${ }^{161}$ In an attempt to better understand how pages differ, I have catalogued the number of different elements per page in the table below.

\begin{tabular}{|c|c|c|c|c|c|c|c|c|c|c|}
\hline & I & II & III & IV & V & VI & VII & VIII & IX & $\mathbf{x}$ \\
\hline events & 28 & 29 & 23 & 36 & 34 & 42 & 26 & 33 & 20 & 35 \\
\hline pitches (on staff) & 11 & 8 & 5 & 11 & 9 & 0 & 0 & 0 & 1 & 0 \\
\hline non-specific tones & 20 & 40 & 36 & 30 & 48 & 52 & 60 & 49 & 38 & 54 \\
\hline microtones & 2 & 1 & 1 & 0 & 2 & 0 & 0 & 0 & 0 & 0 \\
\hline diagonal lines (incl. rests) & 16 & 15 & 13 & 29 & 22 & 14 & 13 & 20 & 0 & 20 \\
\hline horizontal lines (incl. rests) & 9 & 13 & 14 & 12 & 17 & 17 & 15 & 17 & 14 & 16 \\
\hline rests (---x:0---) & 0 & 1 & 6 & 8 & 7 & 3 & 2 & 7 & 3 & 4 \\
\hline coordination delays (--- x) & 4 & 5 & 0 & 5 & 1 & 4 & 3 & 8 & 0 & 8 \\
\hline cues from other players/environment & 2 & 3 & 1 & 4 & 3 & 0 & 5 & 0 & 0 & 0 \\
\hline vertical lines & 5 & 13 & 14 & 7 & 15 & 19 & 14 & 9 & 8 & 5 \\
\hline ties & 7 & 14 & 8 & 2 & 14 & 8 & 13 & 4 & 5 & 6 \\
\hline multi-tones (black \#) & 0 & 0 & 0 & 0 & 0 & 0 & 0 & 4 & 3 & 6 \\
\hline simult. multi-tones (black \# in bracket) & 0 & 1 & 0 & 0 & 2 & 0 & 0 & 0 & 0 & 0 \\
\hline multi-timbres (red \#) & 2 & 1 & 5 & 13 & 8 & 1 & 5 & 11 & 9 & 12 \\
\hline non-spec. changes (black \#) & 6 & 6 & 4 & 4 & 3 & 2 & 2 & 0 & 1 & 0 \\
\hline timbral changes (red \#) & 5 & 7 & 3 & 3 & 1 & 3 & 4 & 2 & 1 & 0 \\
\hline specific transformations (arrows, etc.) & 2 & 0 & 0 & 0 & 3 & 6 & 3 & 4 & 3 & 0 \\
\hline anything (x) & 3 & 1 & 0 & 1 & 1 & 0 & 0 & 2 & 0 & 0 \\
\hline high/low (attached arrows) & 1 & 4 & 1 & 0 & 3 & 0 & 4 & 3 & 0 & 0 \\
\hline dissonant/far/middle & 3 & 1 & 0 & 2 & 2 & 0 & 0 & 1 & 0 & 0 \\
\hline noise & 2 & 1 & 1 & 2 & 1 & 0 & 0 & 0 & 0 & 0 \\
\hline harmonics & 0 & 0 & 0 & 0 & 0 & 1 & 0 & 0 & 0 & 0 \\
\hline physical materials/gestures & 0 & 0 & 0 & 0 & 0 & 23 & 13 & 13 & 8 & 23 \\
\hline
\end{tabular}

Table 4. Wolff, For 1, 2 or 3 People. Statistical data

161 Thomas DeLio, Circumscribing the Open Universe (University Press of America, 1984), 62. 
This table illustrates structural patterns that seem to indicate Wolff's poietic strategies for increasing openness in his composition. For example, the first five pages have more specifically notated pitches than the last five and consequently contain more instances of markings associated with pitched instruments, such as microtones, dissonance, noise. They also feature more non-specific changes over horizontal legato lines and explicit cues from other players (though cues exist on every page except IX). The last five pages seem to be more suitable for percussion instruments as there are less indications of pitch and more indications of physical materials and gestures. Clearly, the score is designed to accommodate many different modes of sound production and, by extension, a broader range of potential performance contexts.

Like the I-Ching, For 1, 2 or 3 People is ergodic in that the textual machine requires the performer to determine the configuration of scriptons. This piece is guided by a "class of goals" (as Eno suggests), and it exists somewhere near the middle of the compositionimprovisation continuum; the ergodic design makes it less determinate ("a particular goal"), and the careful decisions made about the specificities of the score make it less indeterminate ("goalless behaviour"). ${ }^{162}$ However, while the score for For 1, 2, or 3 People could be considered ergodic, it is not cybernetic.

Using Aarseth's typology, For 1, 2 or 3 People could be classified in the following manner:

1. Dynamics - static (texton and scripton quantity are preserved)

2. Determinability - indeterminate (scripton adjacency is variable via page selection

162 Eno, "Generating and Organizing Variety in the Arts," Audio Culture, 227. 
and choices about event sequence)

3. Transiency - intransient (passing of time has no effect on the score)

4. Perspective - impersonal (user does not identify as an agent within the score)

5. Access - controlled (via rules, not mechanism)

6. Linking - none

7. User Function - configurative (includes explorative and interpretative)

Because the notation used is somewhat ambiguous, the interpretative user function is extremely important in determining the actual musical result. (As previously stated, all music notation is at least partially ambiguous since notation cannot possibly account for every single aspect of sound.) The elements of this piece, including all of its ambiguities, were careful chosen by Wolff and he addressed the inclusion of ambiguous notation in the following manner:

I often have the experience of performers being confronted with these scores, and trying them out, and then really complaining... "Why do I have to do this? This doesn't make any sense. Is this the way you wanted it?" And they just have to wrestle with it. Especially the question "Is this the way you wanted it?", which I always evade, just on principle. ${ }^{163}$

For 1, 2 or 3 People possesses many characteristics of games as they have been defined in Chapter 1, such as goals, rules, and voluntary participation are all present. A feedback system is not intrinsically part of the artifact, though feedback does affect score traversal since performers often wait for external cues before playing an event. The presence of a quantifiable outcome or a solvable problem is debatable, but certainly a conflict or struggle to follow the rules of the score exists. To defy such rules is to violate the magic circle and operate outside of the boundaries of the piece. One important quality of games

163 Christian Wolff in R. Carl, "Christian Wolff: On Tunes, Politics, and Mystery," Contemporary Music Review, vol. 20, no. 4 (2001), 64. 
that was evidently considered by Wolff is surprise. While it's true that performers may choose their materials and paths ahead of time, the complexity of the score is such that cues could potentially align differently from performance to performance, which would change the overall result. Furthermore, in the case of only one performer, sounds from the audience or the environment may act as cues and thus affect how the artifact is traversed, which adds an extra element of surprise to an already complex structure.

Despite its ergodic properties, the score for For 1, 2, or 3 People is like most traditional scores in that it operates almost entirely on rules rather than affordances. If anything, the printing of each movement on one sheet of paper affords easy configuration on a macro level (like hypertext or the selection of stages in Guitar Hero). However, any violation of the performance instructions is met only by expulsion from the magic circle of play.

\section{Screen Scores}

At the turn of the 21 st century, composers began employing computers to create dynamic visual scores, also known as animated scores or screen scores. Like hypertexts, screen scores dynamically control the presentation of information to the player. As Cat Hope and Lindsay Vickery point out, the enterprise is not entirely new, with similar experiments by Kandinsky, Scriabin, and Stockhausen dating back to the beginning of the century. ${ }^{164}$

164 Cat Hope and Lindsay Vickery, "Screen Scores: New Media Music Manuscripts," Paper presented at the annual meeting of the International Computer Music Conference, University of Huddersfield, UK, 31 July - 4 August 2011. 
But since computers have become smaller, faster, and cheaper, they have also become more generally accessible and more useful for such a purpose. As Aarseth explains, computers offer an unprecedented level of automation and speed when storing and accessing information, which for the first time "breaks down concepts such as 'the text itself' into two independent technological levels: the interface and the storage medium." 165 Screen scores allow for dynamic musical notation with respect to movement (e.g. scrolling), permutation, transformation (e.g. scaling), and generation, and such change can happen practically instantly. ${ }^{166}$

Screen scores are mechanically cybernetic, and thus have the potential to exhibit various behavior given various input. Such a capability affords the element of surprise, an important quality of games and playful behavior. In other words, it is a way to intentionally introduce noise into a system to provide new information. Another benefit of screen scores is the potential to easily display the traversal of the score publicly via projection, video monitors, or even personal electronic devices, allowing the audience to "more readily comprehend the score's relationship to its musical interpretation." 167

\section{Case Study: Ryan Ross Smith, Study No. 46 (2014)}

Ryan Ross Smith is a prolific composer of what he calls Animated Music Notation or AMN,

\footnotetext{
165 Aarseth, Cybertext, 10.

166 Hope and Vickery, "Screen Scores," 226-8.

167 David Kim-Boyle, "Real-time Score Generation for Extensible Open Forms," Contemporary Music Review, vol. 29, no. 1 (2010), 5 .
} 
creating approximately fifty works in this format for various ensembles since 2010. After seeing the the work of Icelandic composer Gudmundur Steinn Gunnarsson and the S.L.A.T.U.R. collective, Smith was inspired by "the rhythmic complexity [Gunnarsson] was able to achieve with such simple means."168 In his paper, "An Atomic Approach to Animated Music Notation," Smith highlights some salient qualities of AMN such as "the actualization of contact and intersection, which provide perceptible indications as to the specific temporal location of sonic events," and the fact that "the dynamic score has agency over the performer; movement is perceptible not of the eye, but to the eye." Quoting Vickery, he makes the case for "semantic soundness" and the importance of "extensible clarity over verbose ambiguity." 169

In his Study No. 46, twelve vocalists follow attack cursors (six per side, for the sake of legibility) that travel on curved lines. (Figure 34) These attack cursors indicate vocalizations between any 2 of 43 phonemes. Phonemes are not repeated and paths are generated randomly with each performance. Rate of change is also determined randomly between a defined range of 1200-2000 milliseconds. He explains, "This range is deliberately small, but with enough micro-variation that the general density is preserved, but irregular. I generally refer to this as a sense of consistent-inconsistency."170 In describing the concept for the piece, Smith says, "I wanted to write a piece for a choir,

\footnotetext{
168 Ryan Ross Smith, email message to author, September 17, 2015.

169 Ryan Ross Smith, "An Atomic Approach to Animated Music Notation," Tenor 2015, Proceedings of the the International Conference on Technologies for Music Notation $\&$ Representation, Universite Paris Sorbonne/IRCAM, Paris, 2015.

170 Smith, email message.
} 
wanted to use text, but did not want to be responsible for the implications (narrative, etc.) of that text. By using the sonic building blocks of language, text is only a potential, its actualization as anything meaningful a mere byproduct of its functionality." 171

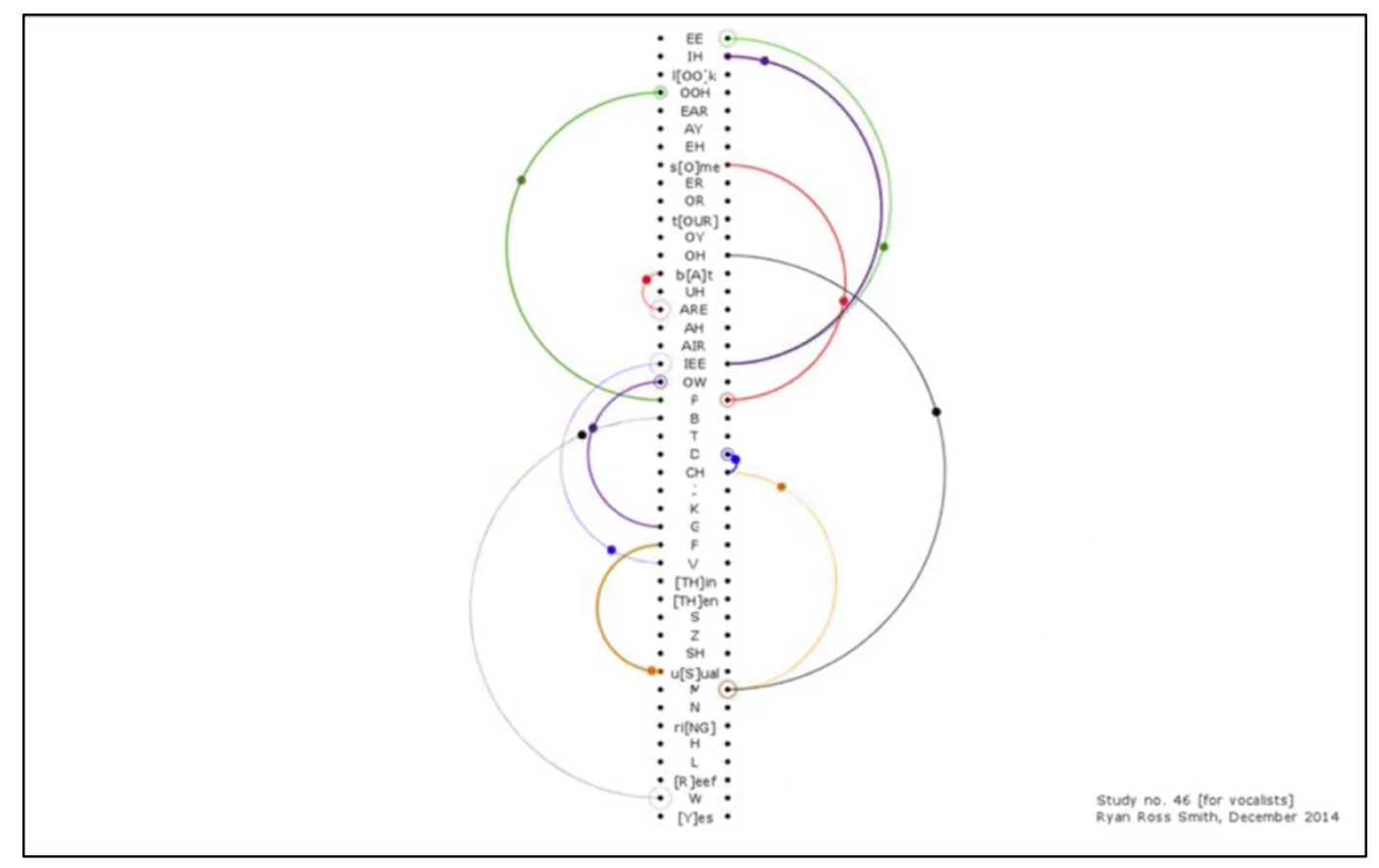

Figure 34. Smith's Study No. 46

\section{Case Study: Paul Turowski, $S Q(2012)$}

I composed $S Q$ for the Voxare string quartet, a group of talented musicians with excellent sight-reading skills, keen musical memory, and mastery of traditional performance practice. I decided to draw upon these strengths by exploring the potential of animated

171 Ibid. 
digital scores. ${ }^{172}$

On a fundamental textonic level, the performed artifact of SQ exists as a fixed score. (Figure 35) Standalone software, created in the game development environment Unity, regulates access to images of the fixed score in a randomized fashion. Pitches that are permissible to play (according to the rules) are indicated by an overlapping active window; upcoming pitches are visible to the right of the active window, but they are slightly grayed out. The active window resizes and moves over the course of the piece, indicating anywhere from one to seven pitches in length at a time.

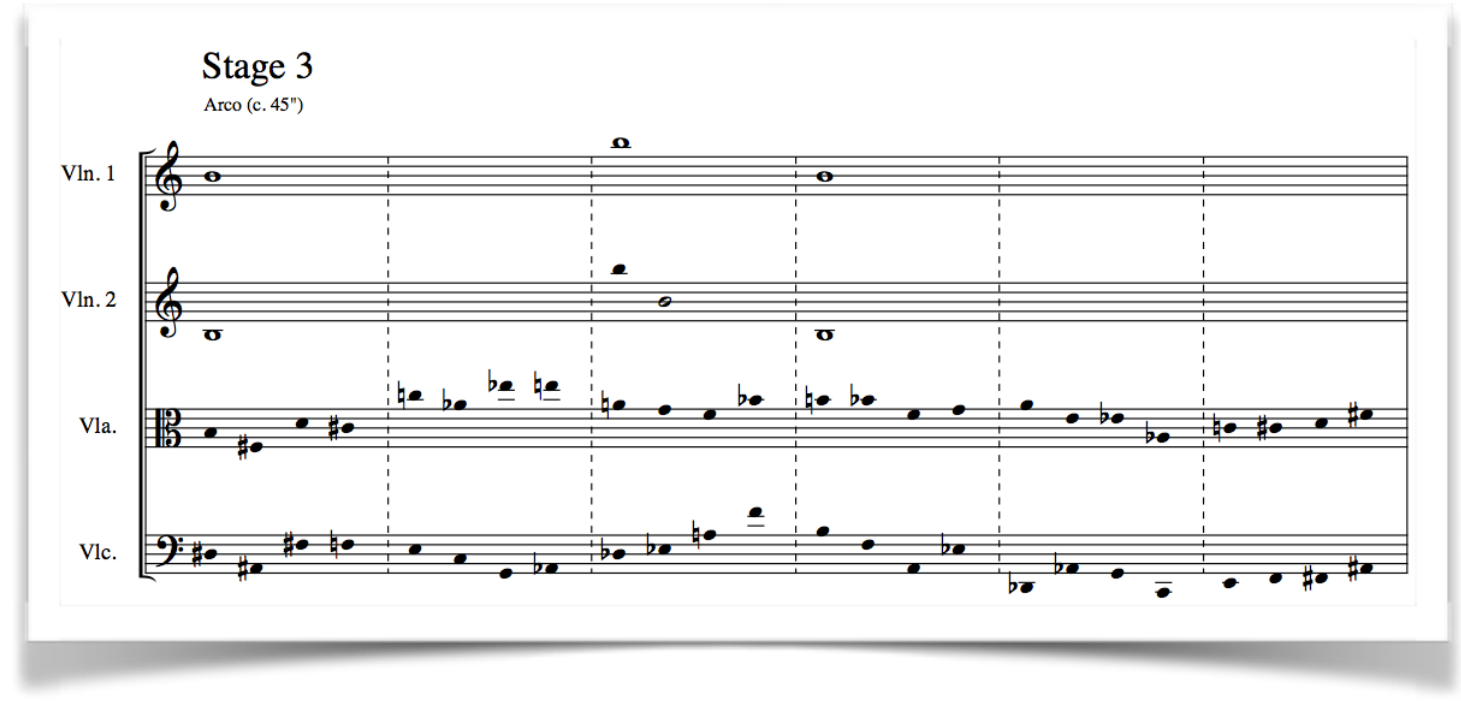

Figure 35. Turowski, SQ. Fixed score component: pitch set for Stage 3 of 5

In addition to pitch, several other parameters are specified by the animated score. Onsets

172 A video of a realization by the Voxare quartet can be viewed at: https://www.youtube.com/watch?v=Aax5Vg69Yh0 [accessed 2016MAR02]. 
are indicated redundantly by 1) a flashing orange bar at the top of the active window, 2) the movement of an object that resembles a bow, and 3) the collision of colored dots with the center of this screen. Moments of temporal alignment between all players are indicated by simultaneous convergence of the colored dots as well as by the word "ALIGN" in the active window. The outward displacement of the dots indicates relative dynamics in three levels (soft, medium, loud). Additionally, the bow indicates particular types of articulation (arco, ricochet, staccato, and tremolo), both with animated movement (elastic anchor) and text located directly above the bow.

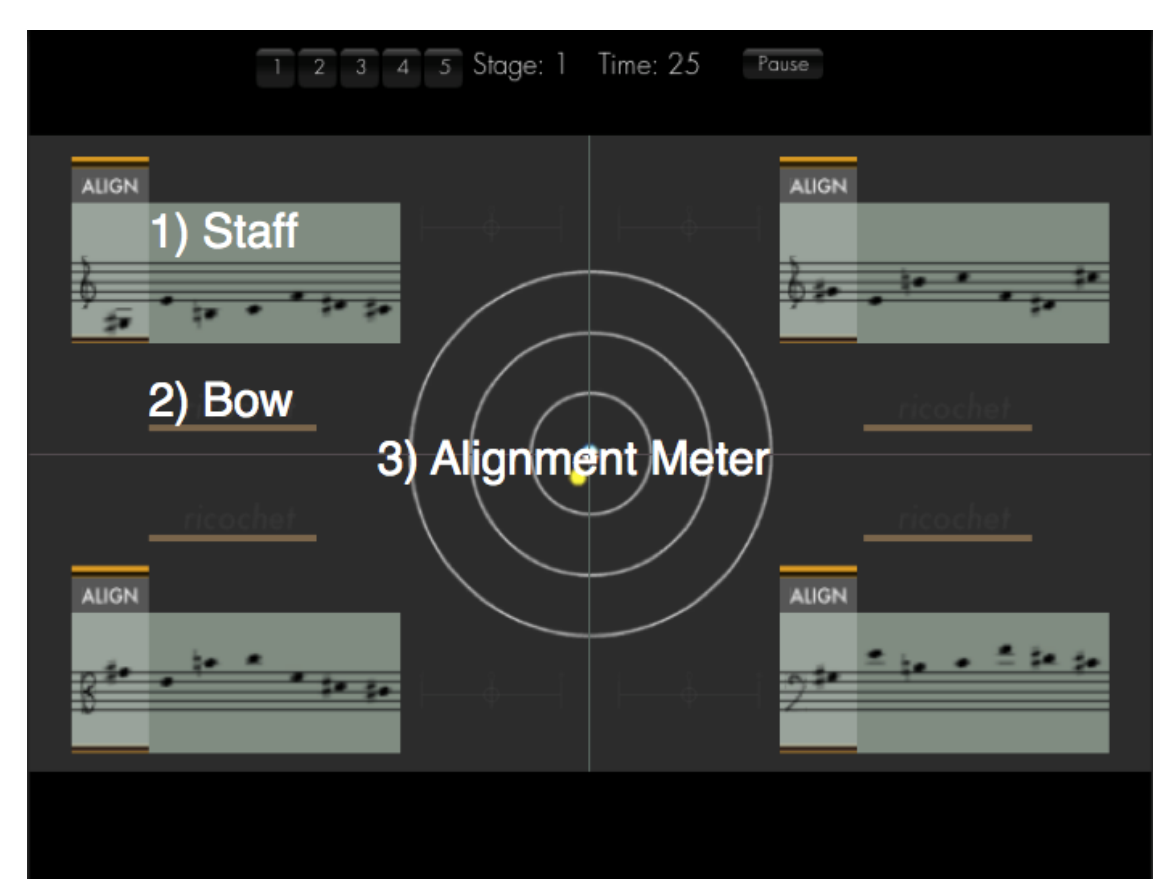

Figure 36. Turowski, $S Q$, Stage 1 screenshot + labels

In Stages 3 and 4, meters appear in each quadrant that indicate relative amounts of detuning to be implemented by performers. A circle at the center of the meter indicates 
no change, a circle at the left end of the meter indicates -50 cent modification and and a circle at the right indicates a +50 cent modification. These detuning meters are also animated and the speed of the animation indicates the speed at which pitches should fluctuate. (Figure 37)

The fifth and final stage marks a return to the more mechanical spirit of the first two stages from the relatively quiet, slow, and meandering character of the third and fourth stages. The piece's intensity peaks as the animated onset circles indicate the fastest tempo of the piece (approximately $120 \mathrm{BPM}$ ), maximum loudness (full radial displacement of center dots), and an unwavering synchronization.

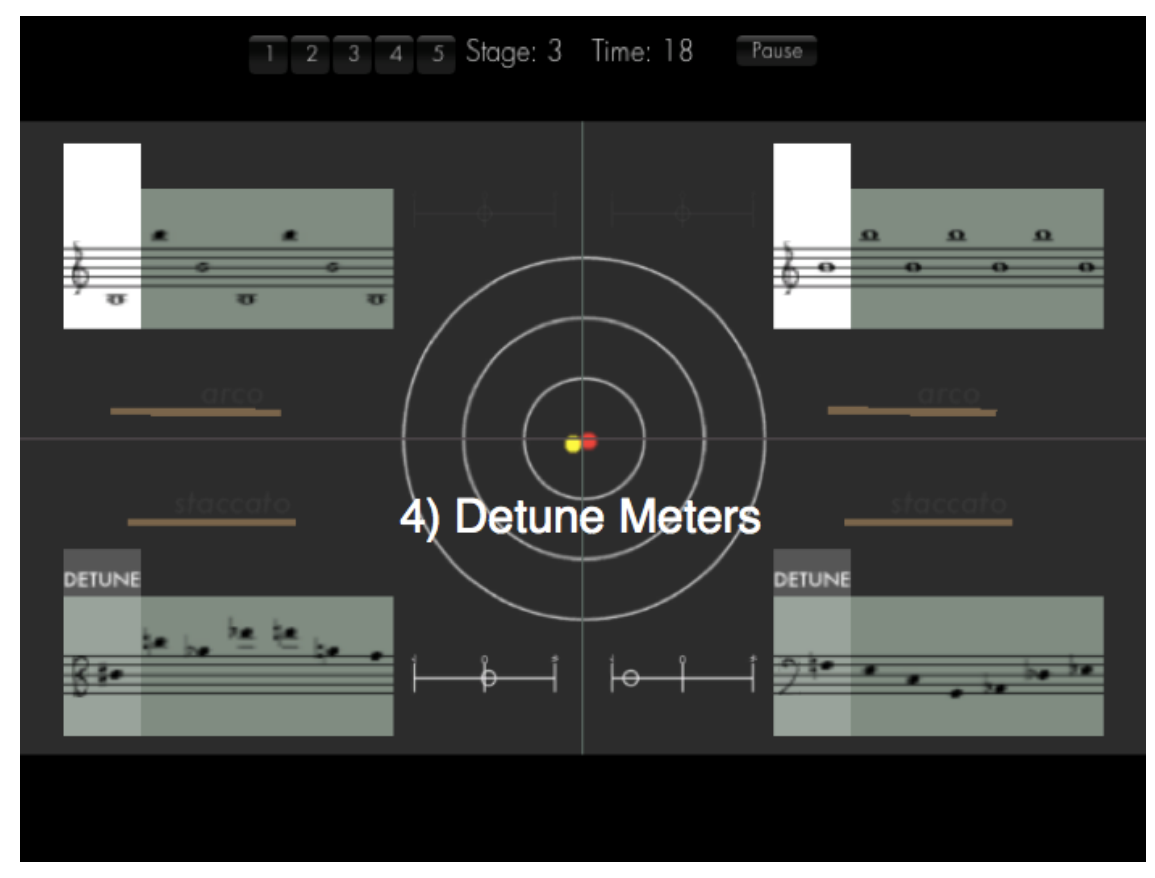

Figure 37. Turowski, $S Q$, Stage 3 screenshot + label 


\section{Aleatoricism}

Both Study No. 46 and $S Q$ rely on random number generators to create new scriptons from composed (i.e. pre-considered) textons. In the case of Study $\mathcal{N}_{0}$. 46, scripton adjacency (i.e. the 'determinability' attribute of Aarseth's typology) is variable, resulting in a larger possible number of combinations and thus a more surprising outcome. In $S Q$, the performers can rely more on a preconceived path since scripton adjacency remains almost entirely fixed. In general, a performed artifact that utilizes randomness to generate scriptons easily evokes a sense of play (see Caillois's play category of alea), though of course there is also a substantial amount of work involved in learning the nature of the materials and the dynamics of the system. As with Wolff's open score, realizations of Study $\mathcal{N}_{0} .46$ and $S Q$ are primarily guided by rules, which can be broken.

\section{Interactive Artifacts}

Interactive digital scores build upon the principles of screen scores by involving player input and dynamic feedback. One robust manner of enabling score interaction is through the use of common digital interfaces such as buttons and potentiometers. For a traditional instrumentalist, such a manner of interaction is potentially problematic as it is common to have both hands (or more) occupied with the apparatus of the instrument. To some extent, this can be mitigated with careful design of the controller, such as the implementation of foot pedals or other conveniently placed controllers. In any case, a 
learning period is required to acclimate the performer to a modified manner of playing.

Another method of score interaction might be called control sound -i.e. mapping sonic features to control functions. With this technique, performers need not worry about diverting attention away from the native apparatuses of their instruments. In a paper titled “Computer Game Piece: Exploring Video Games as Means for Controlled Improvisation," the authors list several viable sonic parameters for control sound, including dynamics, density, register, intervals, pitch, and rhythms or phrases. ${ }^{173}$ Timbral features like spectral flux are also accessible via spectral analysis libraries like those created by William Brent for the audio programming environment Pure Data $(\mathrm{Pd}) .{ }^{174}$ Additionally, we might consider that derivatives (i.e. the rate of change of a particular parameter over time), derivatives of derivatives and machine learning algorithms (e.g. Brent's timbreID object) could allow for higher level feature detection, which would provide more lower level freedom for the performer.

Jackowski et al. also catalog a number of approaches for performed artifact sound generation. These approaches include event-based sound effects (e.g. stingers), precomposed soundtrack, direct sonification of game state, sonification of game statistics (e.g. points), and usage of game state elements as parameters in generative music.

173 Dariusz Jackowski et al., "Computer Game Piece: Exploring Video Games as Means for Controlled Improvisation," Proceedings of International Computer Music Conference, Athens, Greece, 2014.

174 William Brent, "Research," web page, http://williambrent.conflations.com/pages/research.html [accessed 2016FEB01]. 
According to the authors, these approaches served multiple functions such as providing control feedback, musical accompaniment and a rhythmic framework within which to improvise. ${ }^{175}$

\section{Case Study: Harris Wulfson, LiveScore (2006)}

LiveScore employs of a system of computers linked together in a client-server network. Each performer is assigned to one client computer and reads the notation from a computer screen. (Figure 38) The server computer regulates data flow to and from the client computers and is connected to a control box with potentiometers that members of the audience can manipulate as they see fit. (Figure 39)

At the start of the piece, the various parameters of the music are initialized to their default values and remain static. When an audience member approaches the box, they are presented with a choice to affect lower-level musical parameters such as 'range', 'register', 'dynamics', 'pulse', 'durations' and 'durations range', as well as higher-level factors such as 'sparseness', 'irregularity', 'pitch-i-ness', 'non- stasis', and 'togetherness'. ${ }^{176}$ Any change to these audience-controlled parameters results in a change in notation, which in turn affects the overall quality of the composition.

\footnotetext{
175 Jackowski, "Computer Game Piece."

176 G. Douglas Barrett and Michael Winter, "LiveScore: Real-Time Notation in the Music of Harris Wulfson,” Contemporary Music Review, Vol. 29, No. 1, (February 2010), 59.
} 


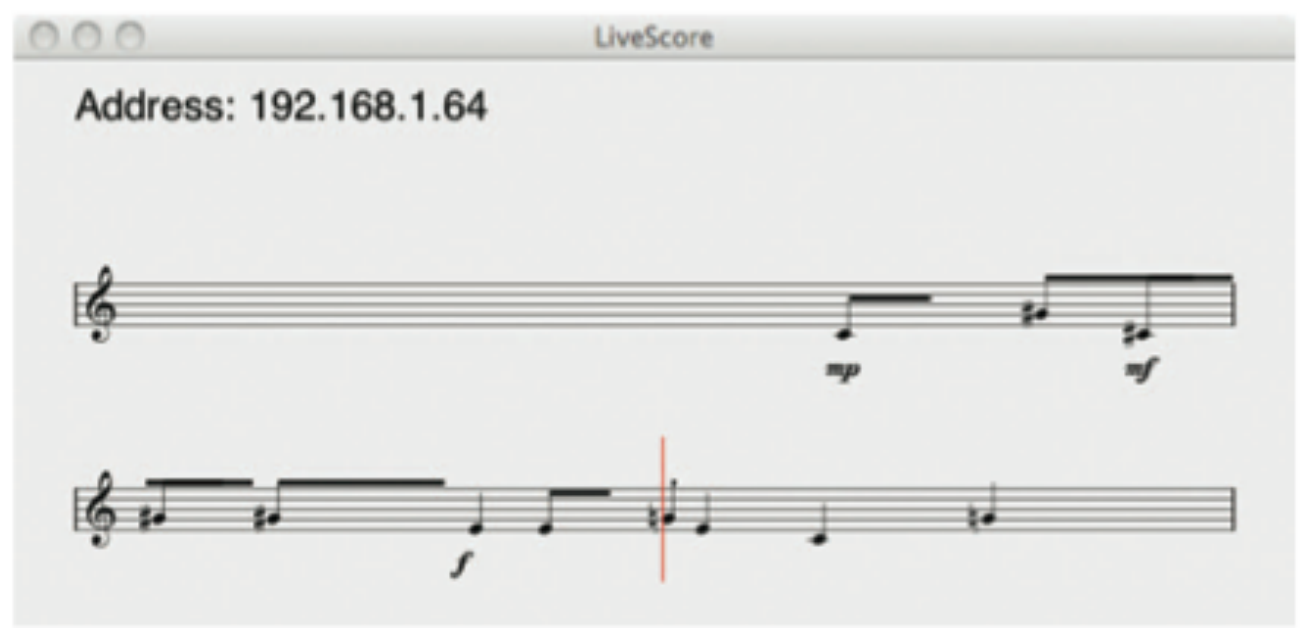

Figure 38. Wulfson, LiveScore. Client interface ${ }^{177}$

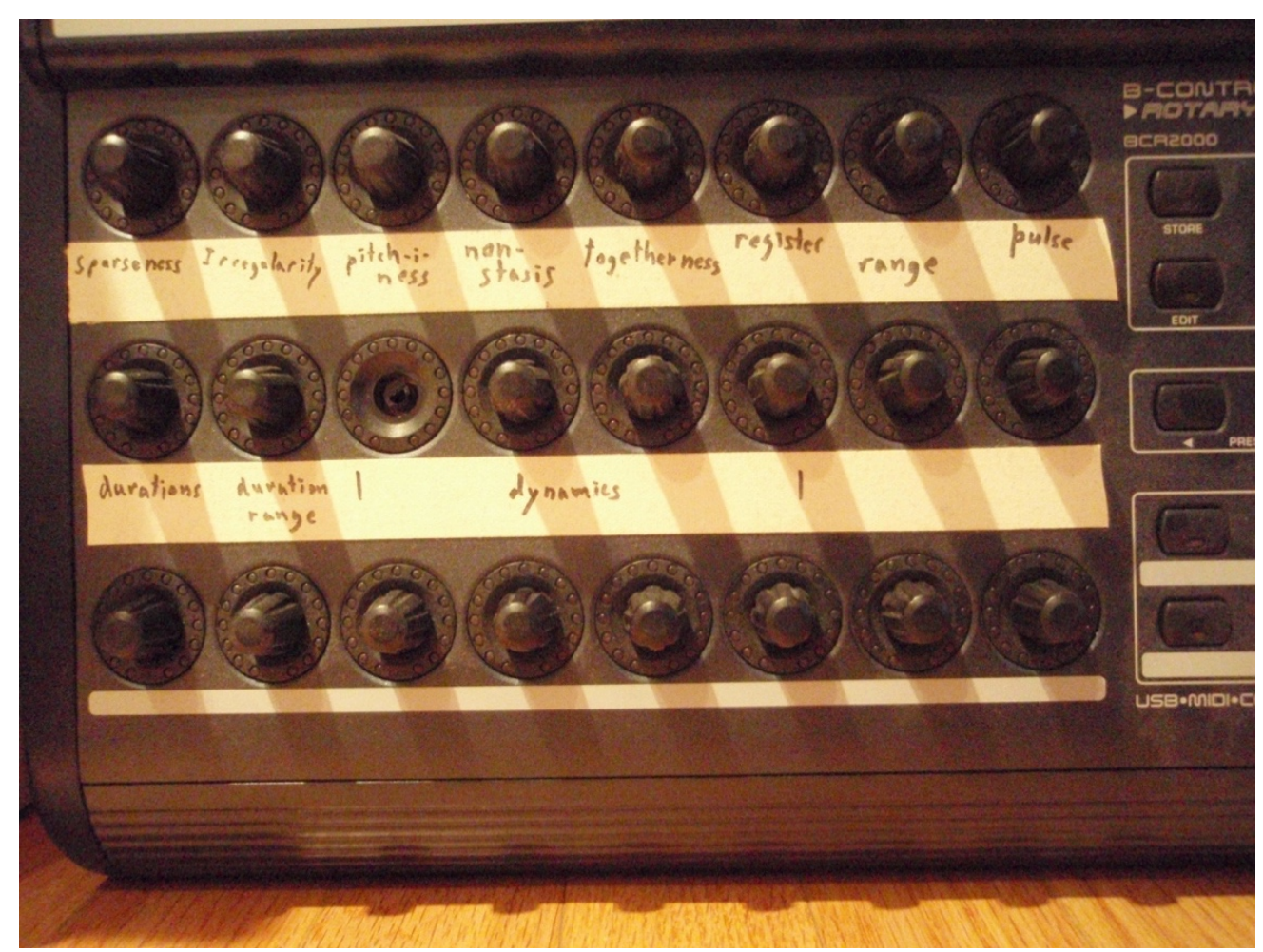

Figure 39. Wulfson, LiveScore. Audience interaction knob box ${ }^{178}$

177 Image from Barrett and Winter, "LiveScore," 61.

178 Harris Wulfson, "LiveScore," http://harris.wulfson.com/?page_id=724 [accessed on 2015SEPT07]. 


\section{Case Study: Joost van Dongen, Cello Fortress (2012-2016)}

Like LiveScore, Joost van Dongen's Cello Fortress makes use of a conventional digital interface - in this case, the video game controller - yet it also implements control sound. It also features a more competitive (Caillois's “agon”) approach to audience interaction than LiveScore. Up to four participants control tanks with game controllers and work together to attack a fortress and gain the high score, while the cellist defends the fortress using musical parameters like pitch, harmony, and melody as controls. For example, fast high notes controls guns, "ugly" dissonant chords control flamethrowers, and low notes set mines. ${ }^{179}$

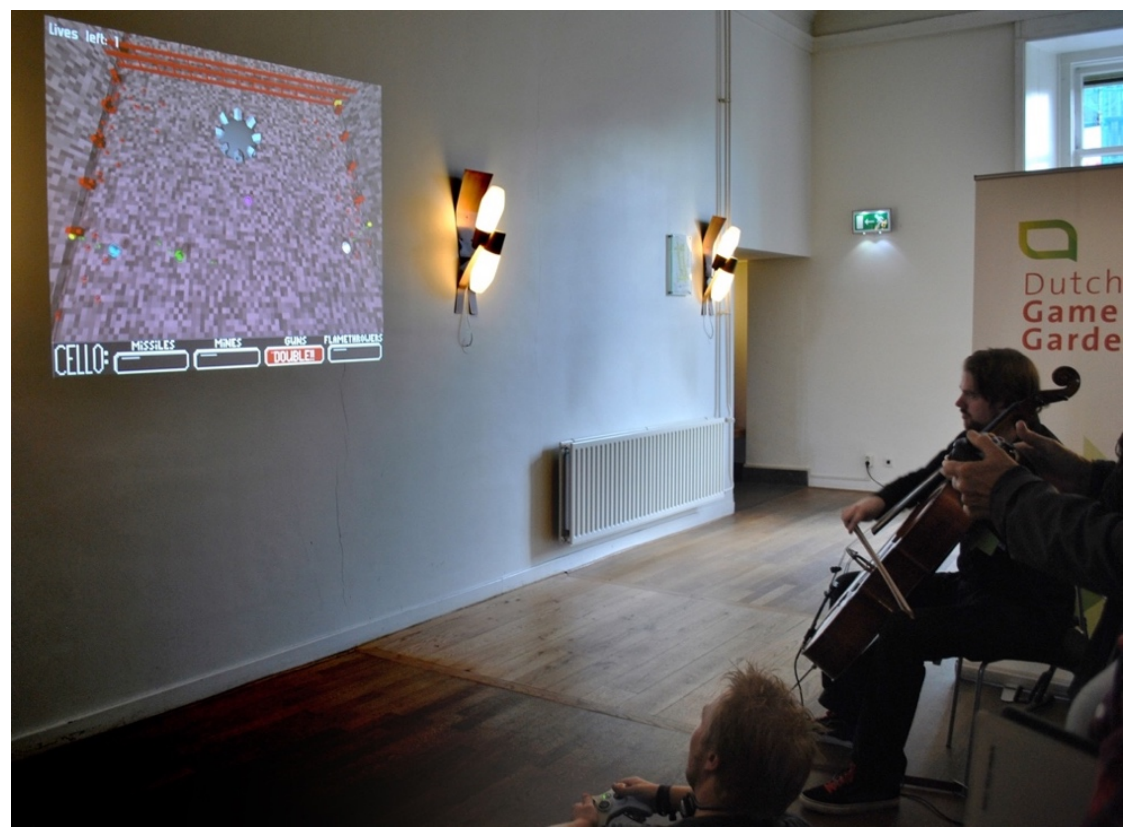

Figure 40. A performance of van Dongen's Cello Fortress ${ }^{180}$

179 Joost van Dongen, Cello Fortress web site, http://www.cellofortress.com/ [accessed 2016FEB01]. 180 Ibid. 
In an email correspondence with van Dongen, he offers more details about the nature of Cello Fortress:

In the end the cello is practically never beaten. I have designed it like this deliberately because this gives the next group a reason to play. If the first group makes it to the end, then the second group is basically going to just play the exact same level again (although with different music since I improvise differently)....

I also take the skill of the players into account: if I play against people who rarely play videogames, I deliberately don't play as effectively to not let them die all the time....

Variation is also important: some attacks are stronger than others but ... I am not trying to win. If I was only trying to win as quickly as possible the game and music would become boring because I would constantly do the same efficient attack. I didn't bother balancing this exactly because my game design assumes the cellist acts as a performer, not as a gamer trying to win. ${ }^{181}$

It is interesting to note that the composer/game designer/performer also functions as a sort of director/conductor who has a significant amount of control over the trajectory of the piece. Such a quality might be compared to performances of Zorn's game pieces, which are usually guided by a leader (usually, the composer himself). Though audience members/players seek to achieve the game-based objective of getting the high score and aren’t necessarily asked to make musical decisions, the cellist does make musical decisions about game length and which musical features to deploy to achieve both aesthetic and gameplay goals. Furthermore, in addition to playing in a way that sounds good and fits the ideal performance duration, the cellist is also taking into account the skill level of the

181 Joost van Dongen, email message to author, June 13. 2015. 
other performers and dynamically adjusting the difficulty level in real-time to maximize the balance of ability to challenge-i.e. flow.

While the cellist and the game players are objectively opposed, they might also be seen as working/playing together to collaboratively form the performed artifact in the same manner as Wulfson's LiveScore. With respect to ergodicity, both artifacts feature configurative user functions that allow all performers (in the case of Cello Fortress, both instrumentalists and participating audience members) to rearrange scriptons, which are comprised of precomposed textons; since performers cannot add new information to the artifacts in real-time, the textonic user function is not afforded. However, a significant ergodic difference between the two pieces is that Cello Fortress makes use of the personal perspective and, by extension, dynamic affordances. Participating audience members identify with the tanks, which can move freely but only in two dimensions and only within the boundaries of the stage. The cellist identifies with the battlements of the fortress, which are stationary but, nevertheless, part of the same pseudo-physical game world inhabited by the tanks.

\section{Digital Game Piece}

Of all the examples presented thus far, Cello Fortress most aptly exemplifies what I call the digital game piece. I use this term rather than "computer game piece"182 for the sake of

\footnotetext{
182 Jackowski et al., "Computer Game Piece."
} 
greater clarity and inclusivity. In the most literal sense, video game refers to a specific type of digital game that is played on game consoles while computer game refers to a digital game that is played on a personal computer. (See Salen and Zimmerman's definition of digital game in Chapter 1.) These terms are often used interchangeably (as demonstrated by Jackowski et al.), and it could be argued that game consoles are also computers, albeit with a specialized function. Nevertheless, such a distinction is potentially useful and thus will be observed in the present context.

The digital game piece is an interactive performed artifact that possesses characteristics of the types of works I have heretofore described, yet in various ways it refines each of them. Like the cybertext, it is ergodic and cybernetic, yet it is primarily musical. Like the open work, it allows more freedom of choice to the player, yet it possesses uniquely designed affordances in a toy-like way. Like the ontological toy, it is designed as a system of play, yet it applies more rules and constraints for the sake of a relatively narrower interactivity and aesthetic outcome. (See Salen and Zimmerman's definition of digital game.) Like the meta-composition, it can procedurally generate a score, yet it is structured with gamebased objectives. Like the digital game, it offers players a world within which to make sound (either acoustically, electronically, or both), yet it is specifically designed to guide external (beyond the artifact) sound production in a concert setting. 


\section{Ghapter 4: Poietic Considerations}

\section{Role of the Author}

Some argue that as constraints are loosened, as a creative work becomes more open, so too does authorship dissipate. 4'33", the (in)famous silent piece, is a prime example of attempted authorial effacement. Through his studies with D. T. Suzuki, Cage was introduced to concepts of Zen, such as the loss of ego, no-mind, being present in the moment, and the "possibility of saying nothing," 183 and allowed these concepts to manifest materially. Similarly, in the world of literature, the author was pronounced dead so that the reader might be born. Barthes notes that Mallarmé was the first to substitute language itself for the person who speaks. ${ }^{184}$ Foucault clarified soon after this idea by stating that "the point is not to manifest or exalt the act of writing, nor is it to pin a subject within language; it is, rather, a question of creating a space into which the writing subject constantly disappears." 185

4'33" may be about loss of ego and saying nothing, but the identity of the composer/author is no less significant as a result. Cage's decision to write out the score

183 John Cage and Lukas Foss, Concerto for Prepared Piano \& Orchestra/Baroque Variations, Liner notes, Nonesuch H-71202, Vinyl LP, 1968.

184 Roland Barthes, "The Death of the Author," UbuWeb, http://ubu.com/aspen/aspen5and6/threeEssays.html\#barthes [accessed 2016FEB01].

185 Michel Foucault, "What is an Author?" The Foucault Reader, Ed. Paul Rabinow, (Michigan: Pantheon, 1984), 102. 
by hand and sign the cover suggests that authorship was at least somewhat important to him. In describing a text score by Stockhausen, Hugh Davis explains that, "one remains aware of the composer influencing the performance from a distance through his score. And the structural indications in the score ... ensure that those elements at least will make the result completely different from a free improvisation." 186 In other words, the influence of authorship is inescapable - even for the most open examples. Perhaps this explains some of the aforementioned comments about jazz by Cage. As Zorn puts it: "I can understand why composers at that time felt compelled to justify their work with intellectual systems and words such as "aleatoric," "intuitive," and "indeterminate." They were trying to justify to the critical community that this was not "improvised music" - music that the performers were making up as they went along - but music that was truly envisioned by a particular mind and then passed down to the performers."187

As with other forms of industrialized entertainment, the creation of digital games is a complex process that often involves large teams of individuals, each with a specialized role. However, it is often the case that they work under a creative director, who guides the collective toward a focused vision of the completed game artifact and, more importantly, the experiences afforded to the player. Works by prominent game directors display recognizable creative styles, as demonstrated by the work of Shigeru Miyamoto (Super Mario Bros., The Legend of Zelda), Goichi Suda (Killer7, No More Heroes), Will Wright

186 Qtd. in Bailey, Improvisation, 80.

187 Zorn, "The Game Pieces," Audio Culture, 196. 
(The Sims, Spore), Peter Molyneux (Populous, Fable), and many more. Even when a so-called auteur is not involved, development teams typically establish a creative identity that guides their output, as exemplified by differences between the detailed open worlds of Bethesda Software titles (Fallout, Elder Scrolls) and the real-time-strategy (RTS) emphasis of Blizzard Entertainment. As is often the case with composers of music, game companies have unique identities that prime player interpretation and expectations.

Authorship is a form of ontological exploration that is related to the aesthetic goals of discovery ("game as uncharted territory") and expression ("self-discovery" or selfarticulation). It might be considered an attempt to find desirable and novel locations in a cultural possibility space - to generate new scripton configurations (e.g. music) from a bank of preexisting textons (sonic resources). Furthermore, performed artifacts like open scores, digital games, and everything between are collaborative authorial experiences. (See Eco's definition of open work in Chapter 1.) As Bartee explains, "authorship allows low-level processes to be combined in unanticipated ways to form complex high-level effects. ... Authorship is extremely important for ontological toys because it allows the player to inject their own philosophical concerns into the toy world, to particularize it in some way by constructing idiosyncratic ontological arrangements." 188

188 Bartee, "Ontological Toys," 74-5. 


\section{A Flat Poiesis}

In conventional Western art music, composers design musical affordances by way of decisions about instrumentation. This includes the employment of extended techniques to expand the field of sonic possibilities. The selection of particular performers and concert spaces also contributes to performance ecology. In other words, affordances are conventionally preset and static, and the role of composer is chiefly concerned with the rule-based organization of these mostly static elements.

The advent of electronic instruments (which decoupled the interface from the soundproducing mechanism) allowed for more various designs of affordance, such as the modular synthesizer - a meta-instrument of sorts. Bartee explains:

Modular synthesizers are not really instruments at all, instead constituting a kind of lego-set or tool box for building instruments. ... Despite its astronomical potential, a disconnected pile of materials and tools does not constitute a musical instrument. From an inordinate number of possible combinations, only a particular instrument must be chosen and constructed. It is exactly this particularity that makes an instrument meaningful. ${ }^{189}$

Nevertheless, the distinction between composer and instrument-builder or score and instrument remains common. Even when considering people that famously both composed music and created new instruments (e.g. Harry Partch), the two roles are usually discussed separately.

189 Ibid., 49. 
By contrast, the composition of a digital game piece involves the simultaneous and relatively balanced consideration of both affordances and rules - i.e. Kanaga's ecologonomy. As Kanaga asserts, "The Form of Games is dualistic-Economic and Ecological, the one dealing with rules which can be broken and changed abstractly, the other dealing with forces which cannot be broken and which can only be changed by concretely reconfiguring the materials conditions which cause them. This is the ground, not goals or optima or anything else - those are economic categories." He also connects this realization to a broader context, stating that, "Ecology is the ground of the economy," and "ignoring this ground is proving deadly on a global scale."190 If a flat ontology places humans and objects on equal ground, then the creation of digital game pieces (as well as other composition-instruments) might be considered a flat poiesis that privileges neither rules not affordances. Since game-based objectives (rules) can sometimes interfere with aesthetic choices (particularly, in a highly improvised context), finding the right practical balance is crucial.

The balanced creation of digital game pieces involves careful consideration of the manner in which subtle changes to game variables can affect the musical outcome. For example, as Smith contends regarding AMN, contact and intersection serve to clearly provide temporal information about sonic events. In game worlds, decisions about the physical properties (e.g. mass, shape, restitution, etc.) of objects directly inform the potential for contact and intersection, and these properties may change over time. Furthermore, the

190 Kanaga, "Intro to Ludic Ecologonomy (Pt. 1)," Wombflash Forest. 
manner in which in-game objects move and react can relate (mis)information to the player as an elastic anchor (e.g. whether an agent is friend or foe).

\section{Didacticism}

Communication (musical or otherwise) is possible through shared codes. Digital games often operate within idiosyncratic systems of logic, employing their own unique symbolic codes and mappings. When presented incrementally and interactively, the rules of the system can usually be learned fairly quickly, regardless of they player's prior experience. For example, in the game Super Mario Bros. a player quickly learns that pressing the A button causes the avatar on the screen to jump. This learned mapping can then be interpolated to other settings to solve puzzles and overcome obstacles. Further, such a basic mapping can be augmented, such as with the acquisition of ability-expanding power-ups. When applied to active scores, this kind of didactic design of affordances can be used to establish strong mappings that can facilitate communication (or miscommunication, as the case may be) of rules. Ben Shneiderman calls this type of interaction "direct manipulation, ... a visual representation of the world of action," which results in the simplification of user tasks. "Direct manipulation is appealing to novices, is easy to remember for intermittent users, and, with careful design, can be rapid for frequent users."191 In other words, it can improve flow and inform the implementation of

\footnotetext{
191 Ben Shneiderman and Catherine Plaisant, Designing the User Interface, $4^{\text {th }}$ ed. (Boston: Pearson/Addison
} Wesley, 2005), 71. 
DDA (see "Success-Failure" in Chapter 2). Additionally, didactic systems that employ physics simulations engage a universal common ground that transcends cultural boundaries. Signifiers (e.g. conventional notation) are, of course, helpful in conveying information to the player too, provided that the signifier uses codes that are shared between the producer and receiver.

\section{Playtesting}

As Salen and Zimmerman point out, digital games often require an iterative design process "because the play of a game will always surprise its creators, particularly if the game design is unusual or experimental. Even a veteran designer cannot exactly predict what will and will not work before experiencing the game firsthand." 192 This iterative process is often referred to as playtesting, and becomes increasingly necessary as the size and complexity of a game increases.

As with any sufficiently open musical work, the experiences afforded by the digital game piece are also difficult to predict. While rehearsal is about smoothing the mechanisms of a performance, playtesting is about smoothing the mechanisms of the performed artifact. (Of course, both can happen simultaneously.) Playtesting serves a practical (fixing bugs) and aesthetic purpose, allowing the composer to shape affordances and constraints so that the desired sonic results are achieved.

192 Salen and Zimmerman, Rules of Play, 12. 


\section{Gase Study: Paul Turowski, Hyperions (2014)}

Hyperions was composed for the Cellotronic Games competition, which was organized by Juraj Kojs and supported by the Foundation of Emerging Technologies and Arts (FETA). It features an animated digital score that combines elements of traditional musical notation with the dynamic quality of digital games to create an interactive context for musical improvisation. Real-time performance decisions about pitch, timing and activity level are recognized by the computer via audio input and influence a physics-based game world. Collisions within this game world act as triggers for the playback and processing of sounds recorded during performance. Chance-based factors, including interactions with non-playable characters (NPCs), allow unique visual and sonic patterns to emerge with each performance. ${ }^{193}$

\section{Game Mechanics and Dynamics}

In Hyperions, the performer creates allies (multi-colored circles) by playing a sustained pitch while the spawner (hollow white circle) is inside one of the three chargers (triangular beams extending out from the center of the playing field). (Figure 41. Note: the embedded image of the performer does not appear on the performer's monitor.) Created allies surf counterclockwise on three ellipses - one for each pitch range - which resize based on total

\footnotetext{
193 A performance video featuring cellist Kevin Davis can be viewed at: https://www.youtube.com/watch?v=ggq49UjScOg [accessed 2016FEB08].
} 
ally energy level per range. An ally's energy (and size) increases whenever it crosses a charger. The outcome of collisions with foes (squares) is determined by relative energy/size. If the ally is equal to or larger than the size of the foe, the foe is destroyed; if the foe is larger, both are destroyed. Allies are connected by springy joints if they collide with each other and are both members of the same chord, which is contingent on the first pitch played and the current stage. There is an additional non-playable character called the freezer (a blue triangle) that darts around randomly and freezes any ally group that touches it. The piece ends once all allies are destroyed (beyond four minutes of elapsed gameplay time; see below).

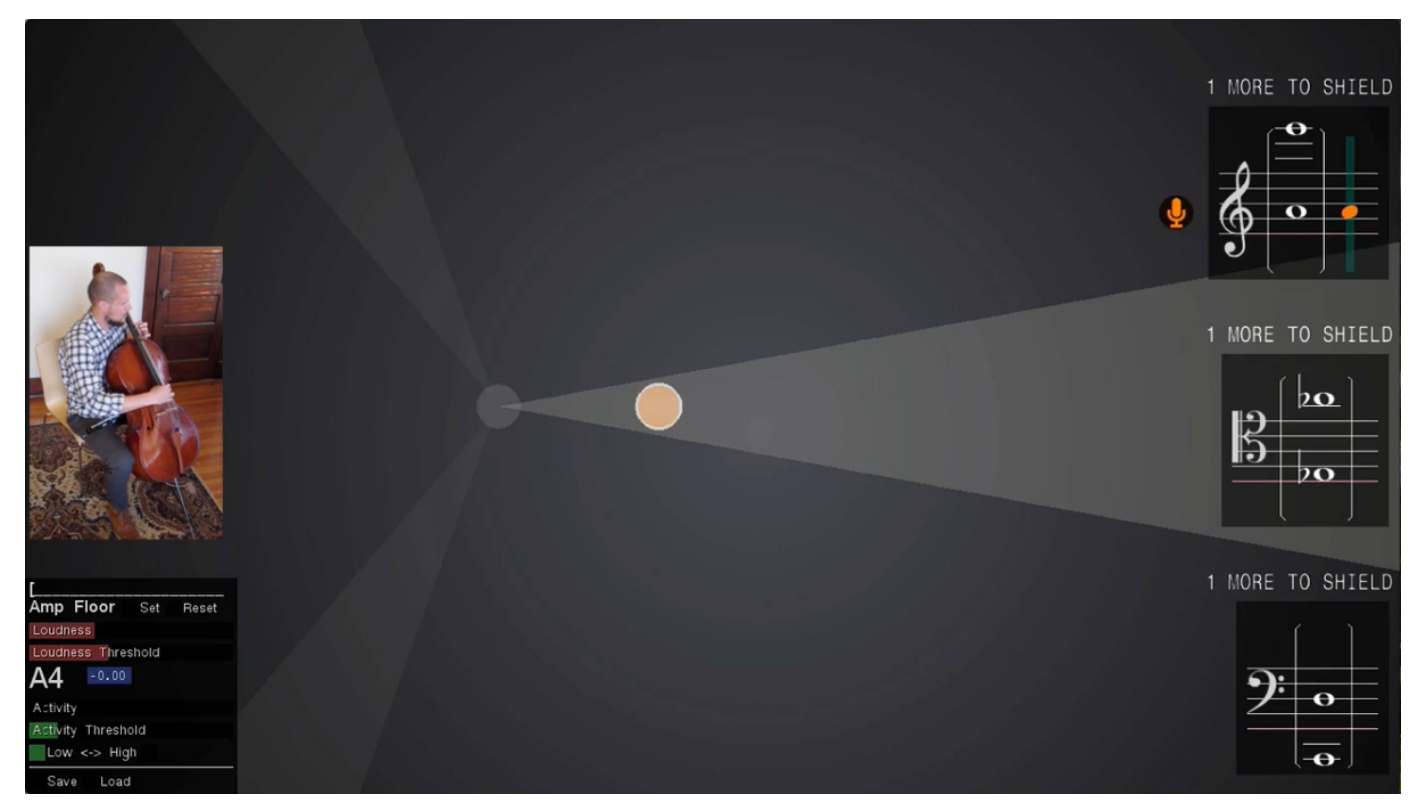

Figure 41. Turowski, Hyperions, Creating an ally

The primary game-based objective of Hyperions is to stay alive against the onslaught of invading foes, but a points-based system also can inform player decisions. Most 
importantly, the performer is asked explicitly (via text-based instructions) to always think musically about their choices rather than just striving for the highest score or longest play time. Ideally, gameplay and music-making should always happen simultaneously, though there may be instances when the the two activities are opposed. In those instances, it's up to the performer to decide which of the two is more important at that particular moment.

\section{$\underline{\text { Performer's Interface }}$}

The visual interface of Hyperions focuses on created allies, which always remain near the center of the screen. As they grow and expand outward, the camera zooms outward to fully encompass them. Additionally, the performer is provided with relevant information via the onscreen graphical user interface (GUI), which includes three musical staves on the right side of the screen and a feature feedback window the bottom left corner. Each staff depicts pitch ranges in brackets as well as colored noteheads that correspond to created ally pitches. If all allies in a particular range are destroyed, the related staff disappears.

\section{Control Sound}

Two channels of audio input are used. A magnetic pickup is used for control sound to prevent audio feedback, while a microphone is used for the recording of audio buffers that play upon particular events and game states. (See "Emergent Garden" below.) Fundamentally, any sounds made by the performer must exceed a preset loudness threshold to serve as control sound. Allies can only be created if the spawner is within a 
charger and if the performer plays pitches that lie within the indicated pitch ranges. Any ally can be shielded (shown as a green polygon surrounding the ally) by playing its corresponding pitch. If a foe collides with a shielded ally, the shield and foe are destroyed while the ally remains intact. (The shield can be reactivated at any time.) As such, pitches corresponding to allies that the player wants to protect might be played more often. Once five allies are created in any pitch range, all allies in that range are given the power to boost in the direction they are facing (shown by a white arrow). Boost can be applied by playing with a high level of activity (i.e. change in amplitude and frequency over time), and allows the player to actively attack, distract, or evade foes.

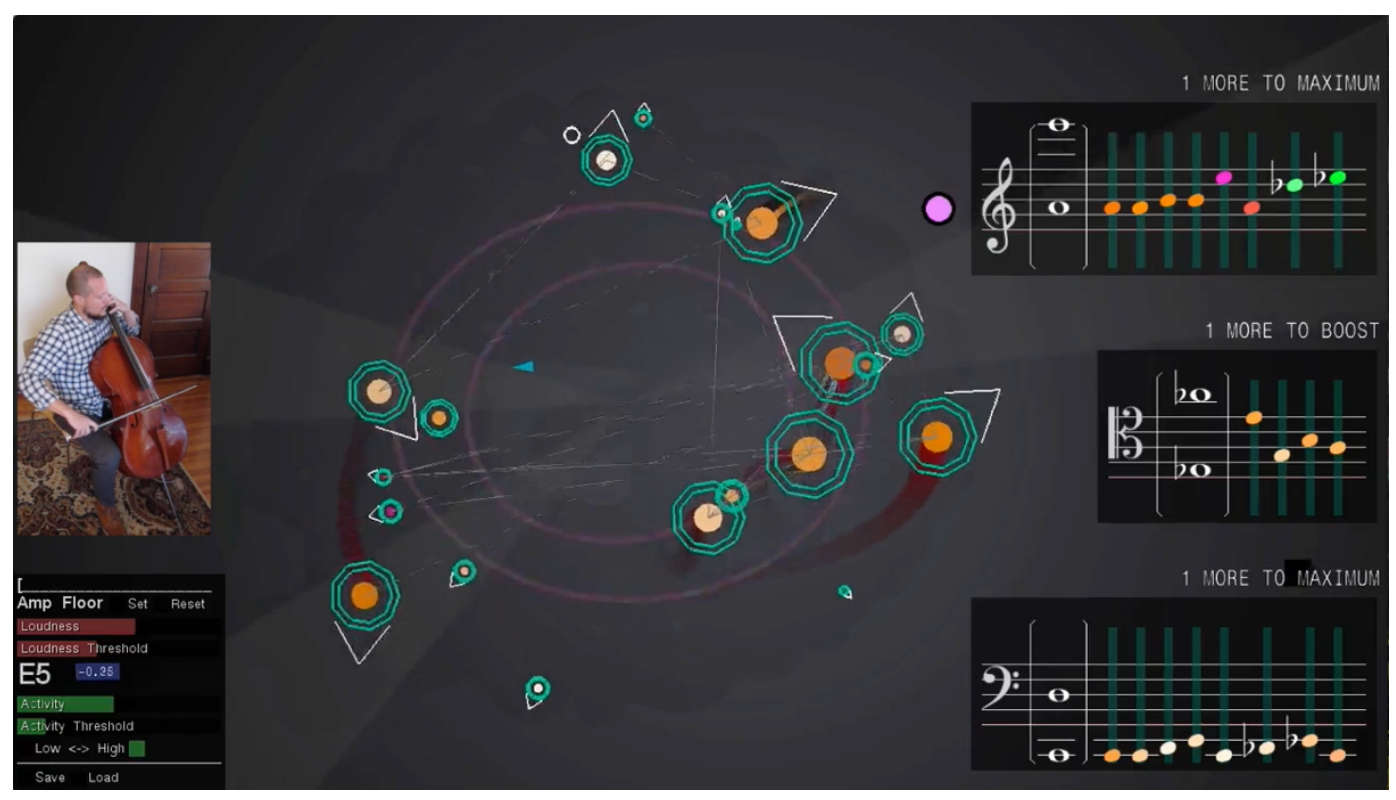

Figure 42. Turowski, Hyperions. Allies boosting

\section{$\underline{\text { Generative Garden }}$}

Allies can be classified as what Kayali calls sound agents, which are "dedicated objects that 
act as audio representations and acoustic attributes. The player can influence partially autonomous objects that act according to the game's set of rules, thus enabling accessible, yet diverse, musical creations." 194 Allies display automatic behavior (e.g. orbiting counterclockwise, bonding together) while also being directly affected by the performer's input. In addition to containing pitch information (that allows mapping of input sound to shield and boost functions), each ally is also mapped to the playback of sounds that were played and recorded during its creation. These sounds play any time an ally crosses a charger beam (i.e. event-based sound effects) or collides with a foe. Given different movement speeds (based on size), boost capabilities, interactions with other agents (e.g. the freezer), and the eventual shifting of charger beams, playback onsets vary widely. Furthermore, these recorded sounds are subject to additional processing (e.g. delay, sample-and-hold) as allies grow in energy and size, and precomposed chordal relationships determine which allies bond together on impact. Since sonic material (textons) can be added to the artifact, Hyperions might be considered to have a textonic user function.

Another generative aspect of Hyperions is the visual design. Ally colors were initially mapped to values of the real-time feature analysis - specifically, bands of bark frequency cepstral coefficients (BFCGs). (Subsequent changes to ally color mapping are discussed below.) As each stage passes, the color scheme shifts to emphasize a different pair of complementary colors. Additionally, ally colors bleed into the background causing

194 Kayali, "Playing Music," 101. 
unique patterns to gradually emerge over time. (Figure 43) This results in a somewhat unpredictable visual stage with each performance, which can potentially influence sound production indirectly via player interpretation.

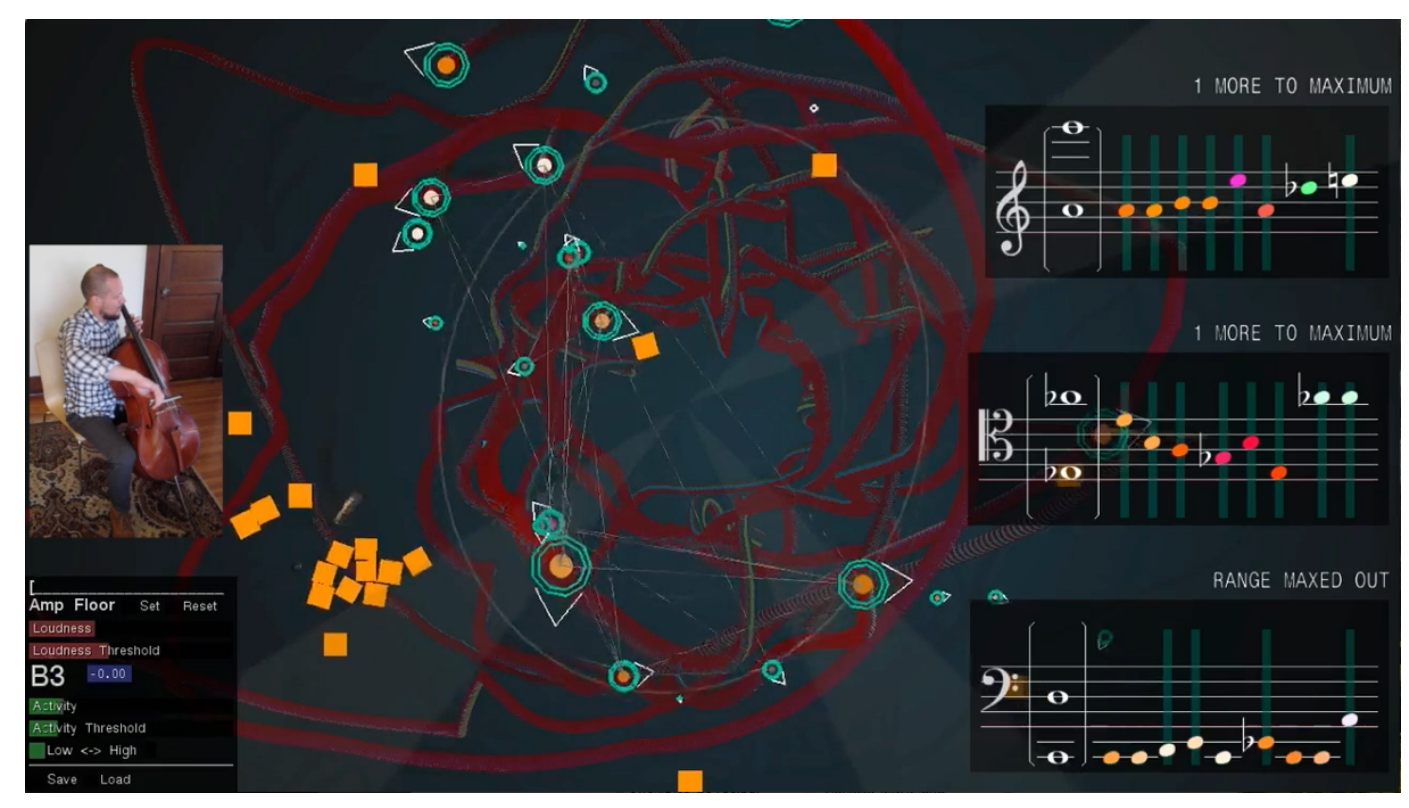

Figure 43. Turowski, Hyperions. Emergent patterns

Despite having an open and emergent quality, many constraints are built into Hyperions which guide the performer toward a specific outcome. For example, when certain ratios of a predetermined goal time arrive, the stage changes. As previously mentioned, stage changes affect the color palette, but they also affect ally binding patterns (and thus chord relationships), as well as foe creation. Foes gradually increase in number over the course of each stage, leading to periodic peaks of intensity. When a stage changes, all existing foes are destroyed, allowing the player to regroup and reassess. Each successive stage produces more foes than the last, resulting in an increasing difficulty curve over time. 
Also, players cannot die until four minutes have passed allowing novice players more time to practice or for unfortunate circumstances to be mitigated. This precomposed and regulated use of time characterizes Hyperions as transient (as defined by Aarseth's ergodic typology).

\section{Playtesting and Revision}

I was fortunate to work with experienced cellist and improviser Kevin Davis to develop this piece iteratively over several rehearsals and performances, and some significant revisions were made as a result of our collaboration. Colors were remapped from the relatively arbitrary BFCGs to pitch - i.e. red (low pitch) to violet (high). Similarly, notehead size was matched to the energy/size of the ally. (Figure 44) These changes improved the interface greatly and allowed Kevin to more easily and more quickly form conceptual blends. Additionally, smoothing values for feature analysis parameters were tweaked to make control more responsive and immediate. Lastly, the difficulty level (i.e. the slope of foe generation) was tuned to heighten flow and to more consistently achieve an average duration of eight minutes.

All of the decisions made about the affordances and rules of Hyperions served to refine the potential outcomes of the performance. While the piece is largely open-ended and heavily determined by the choices of the performer, there is a consistent sequence of events that is predictable for both performer and audience. This consistency is practically useful for the purposes of programming such a piece on a concert, and also contributes to 
a stable identity for the work. Additionally, playtesting served to refine the mechanism of the score and ultimately facilitated a more immersive performative experience.

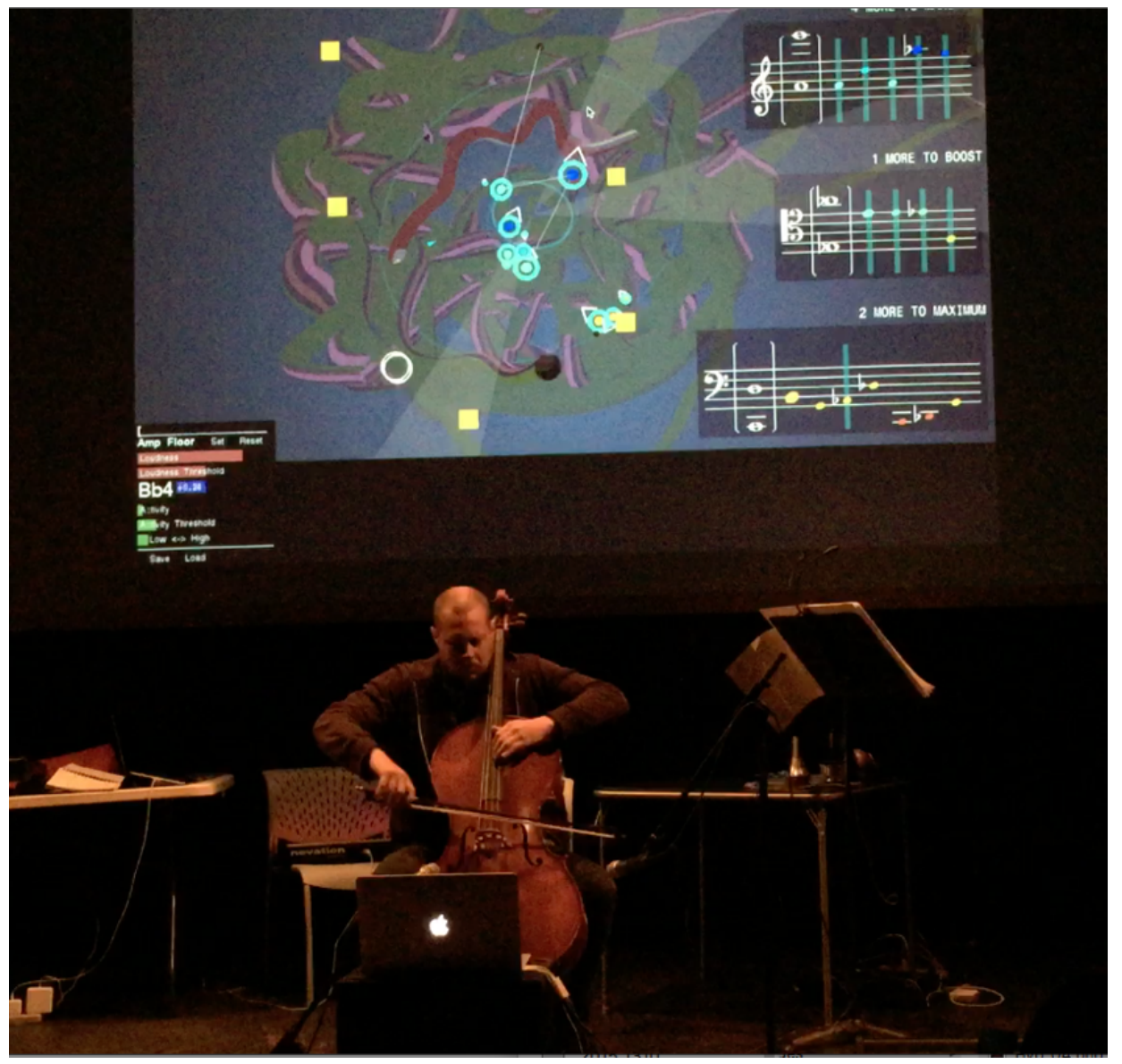

Figure 44. A performance of a revised version of Hyperions at SEAMUS 2015 


\section{Chapter 5: Frontier and Beyond}

Frontier is a digital game piece in which musicians improvise together while collectively influencing an avatar in its journey to the edges of a digital world. Sounds made by the performers are picked up via microphone and particular features like event density, consonance, and spectral flux are mapped to game functions like avatar movement and interaction with NPCs (non-player characters). Primarily, the game/score software serves to provide a dynamic framework for musical improvisers, guiding musical choices with game goals such as exploration, survival, and high score attainment. ${ }^{195}$

\section{An Imaginary Performance}

When the piece begins, players are presented with a circle surrounded by letters. Playing pitched sound causes several things to happen. The letters become highlighted and slightly larger. Extrusions on the edge of the circle stretch outward from the center, highlighted by bright animated particles. The meter on the left side of the screen comes to life, depicting a moving arrow that corresponds to loudness. As more sound is made the shaded inner region of the meter decreases. When sound stops, the meter replenishes soon after. The number in the bottom right corner of the screen increases and decreases with the event density of played sounds. If the sounds made are noisy, the meter in the

\footnotetext{
195 A performance video featuring members of the Virginia New Music Ensemble can be viewed at:
} https://www.youtube.com/watch?v=pS7LwXBvH8Q [accessed 2016FEB08]. 
bottom left corner of the screen moves to the right and the word "FLUX" is displayed. If the sounds are relatively stable (i.e. sustained pitches) then the meter moves to the left and the word "STASIS" is displayed instead. (Figure 45)

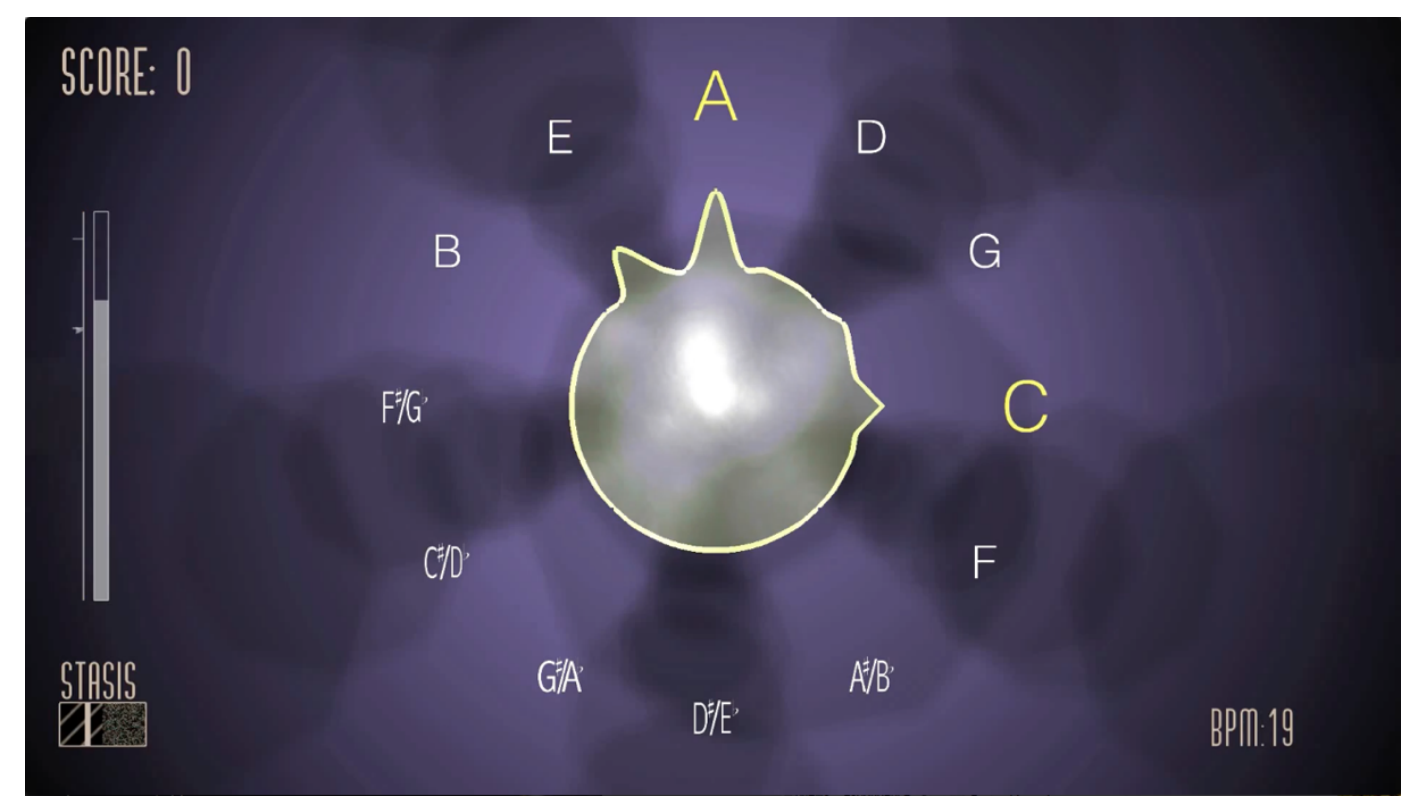

Figure 45. Turowski, Frontier. Opening screen

This opening sequence serves to introduce the players to the rules and affordances that govern control by way of simple direct feedback. Players may also begin to construct conceptual blends based on elastic anchors, such as the meter on the left being a limited energy reserve or pitch serving as a growth vector for limbs on a virtual being of some kind. Direct manipulation establishes correspondences that hopefully align the perspective of the performance collective with this virtual being (the ergodic category of perspective). 
Once the first limb has become fully grown, the camera recedes and players are given more information about their virtual surroundings. The meter on the left and text-based indications remain fixed in position and size, indicating that they are elements of the GUI and not part of the same world that the players' limbed avatar inhabits. Additionally, another circular element appears in the upper right corner.

The avatar is in an enclosure of some kind - a hive of sorts that is inhabited by other beings not directly controllable by sound. They wander aimlessly, and when they collide with the avatar they seem to become agitated, making sounds of their own. They quickly push the avatar away, eventually settle down, and begin to wander once again. Players may notice that each NPC in the hive has a number attached to it, and that playing with an event density that is nearly the same number causes the NPC to react - with a question mark and a change of color (blue to yellow). (Figure 46) If players maintain this correspondence in event density, the NPC eventually turns green and converges with the avatar, which causes a hexagonal icon to appear near the energy meter on the left. (Figure 47) 


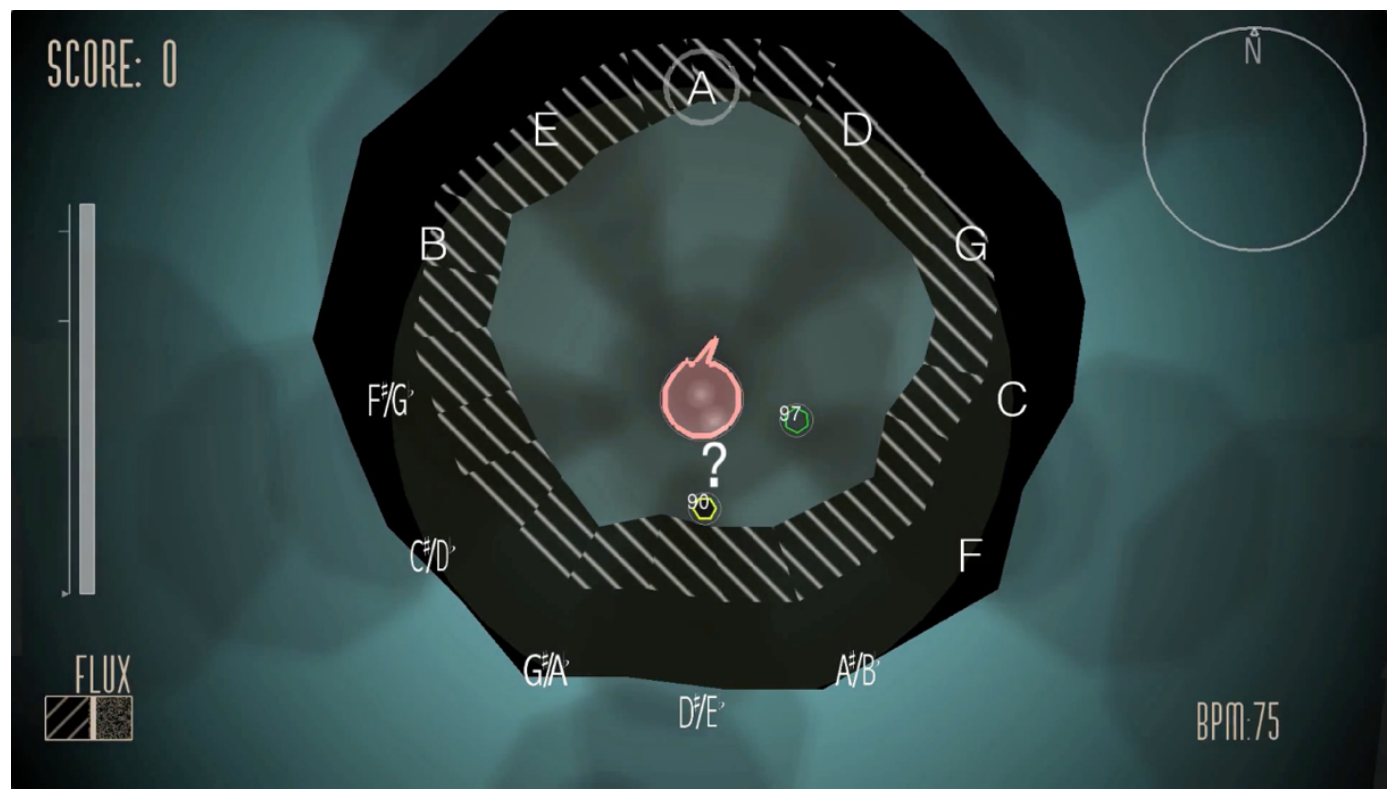

Figure 46. Turowski, Frontier. Encountering NPCs

At this point, playing certain pitches causes the avatar to move in a direction opposite to the corresponding letter. For example, playing an A causes the avatar to move downward. Players may also start to notice that when playing pitches that are consonant with $\mathrm{A}$ (e.g. D or E), the A limb is also affected. If players try to exit the hive, they discover that colliding with the hive's boundaries causes it to gradually turn more and more red. Eventually a block is cleared and the avatar can proceed a bit further outward. Players might also make the connection that patterns on hive blocks match the patterns on the meter in the bottom left. Indeed, more spectral flux allows the clearing of noisypatterned blocks faster, and more stasis allows for faster clearing of striped blocks. As the avatar naturally rotates, so too does the circle in the upper right, providing a compass function. (Figure 47) 


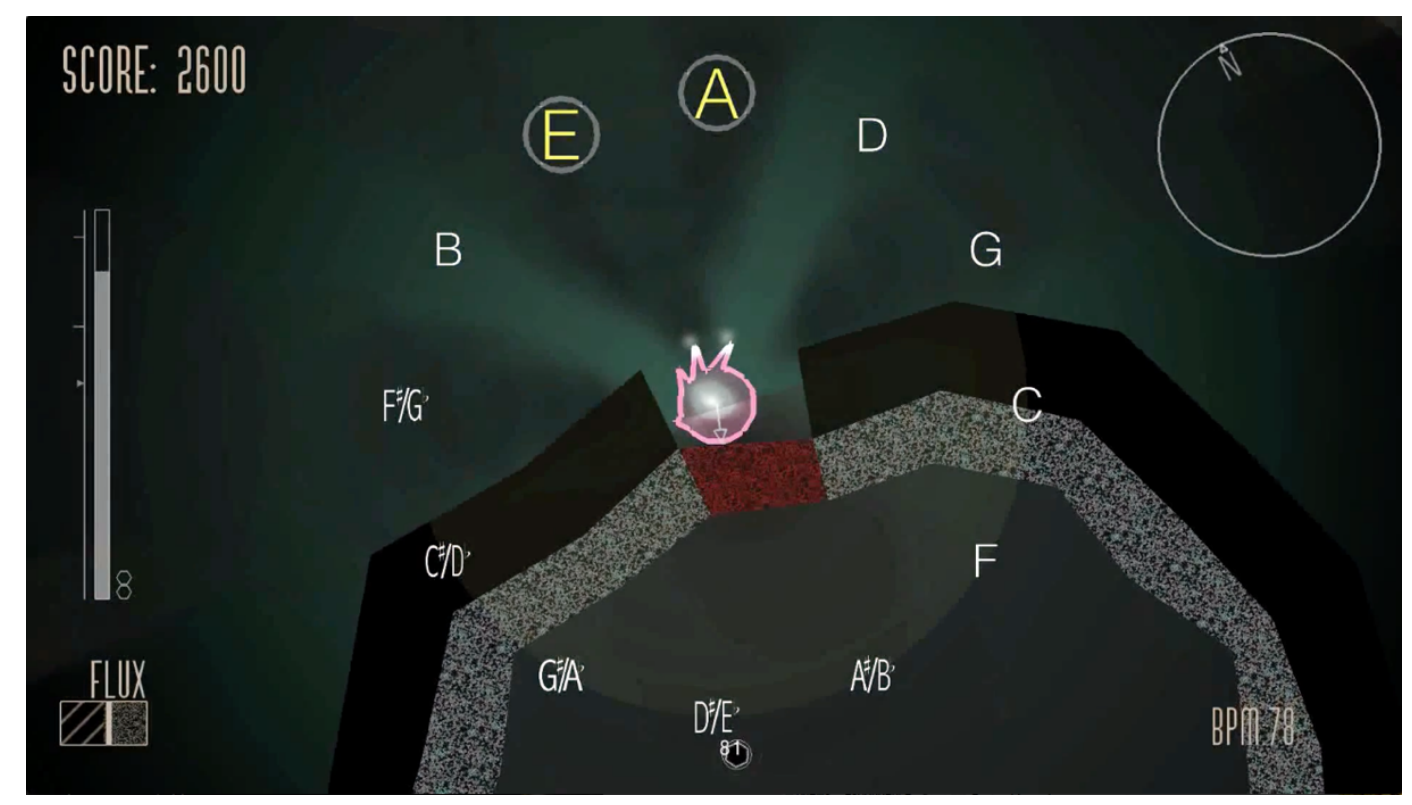

Figure 47. Turowski, Frontier. Breaking hive boundaries

As players venture further outward, they discover that the farther they get from the center the slower their energy meter replenishes. If the energy meter drops too far, the meter turns red and an increasingly loud audio cue plays, warning them of impending danger. Should they completely deplete their energy meter, hexagonal units they have accrued by way NPG joining are expended for a new energy meter. Should they have no hexagonal units left and exhaust the energy meter, the avatar explodes leaving energy particles scattered around the site of the explosion and reappears in the initial hive at the center of the map.

Venturing further outward reveals peculiar gates guarded by NPCs that are larger than others previously encountered. They also behave differently than other NPGs, keeping post at particular positions and attacking when approached. (Figure 48) Out here, the 
compass orientation remains fixed while the pitch letter wheel (that guides movement) rotates.

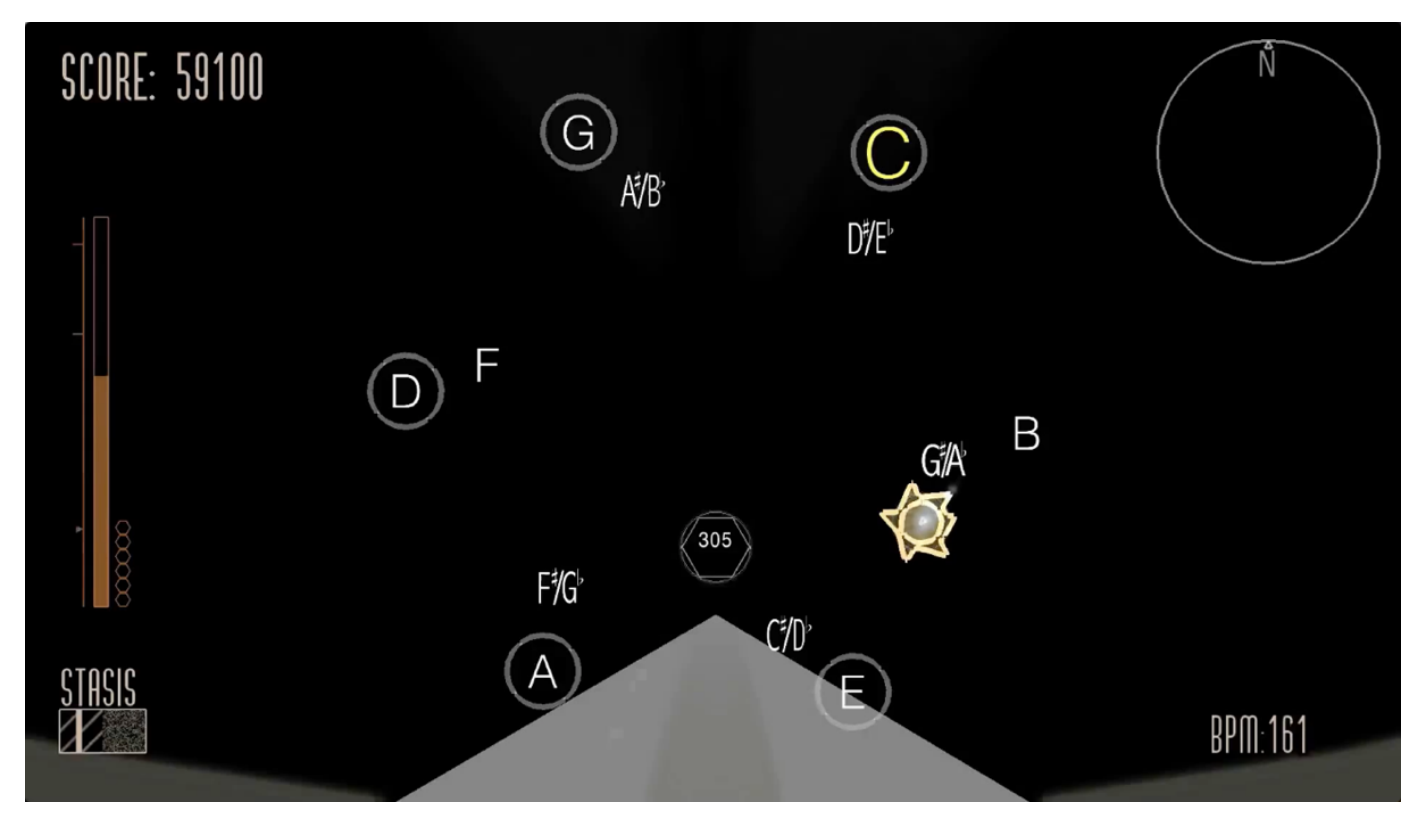

Figure 48. Turowski, Frontier. Discovering gates and guard NPCs

Penetrating the gate (which reddens on collision and eventually dissipates like hive walls) reveals a bleak outer area in which energy does not replenish. (At least the orientation of the pitch wheel is fixed once again.) In this outer area reside peculiar beings that upon collision transport the avatar to a new stage - one which is similarly structured but slightly different. (Figure 49) In this new stage, the avatar has two layers that rotate independently of one another. (Figure 48) In later stages, even more avatar layers are added and dangerous red zones drift around, sapping energy from the avatar. (Figure 50) Players continue to explore the game world until the predefined time limit runs out, or until they have successfully completed all four stages. 


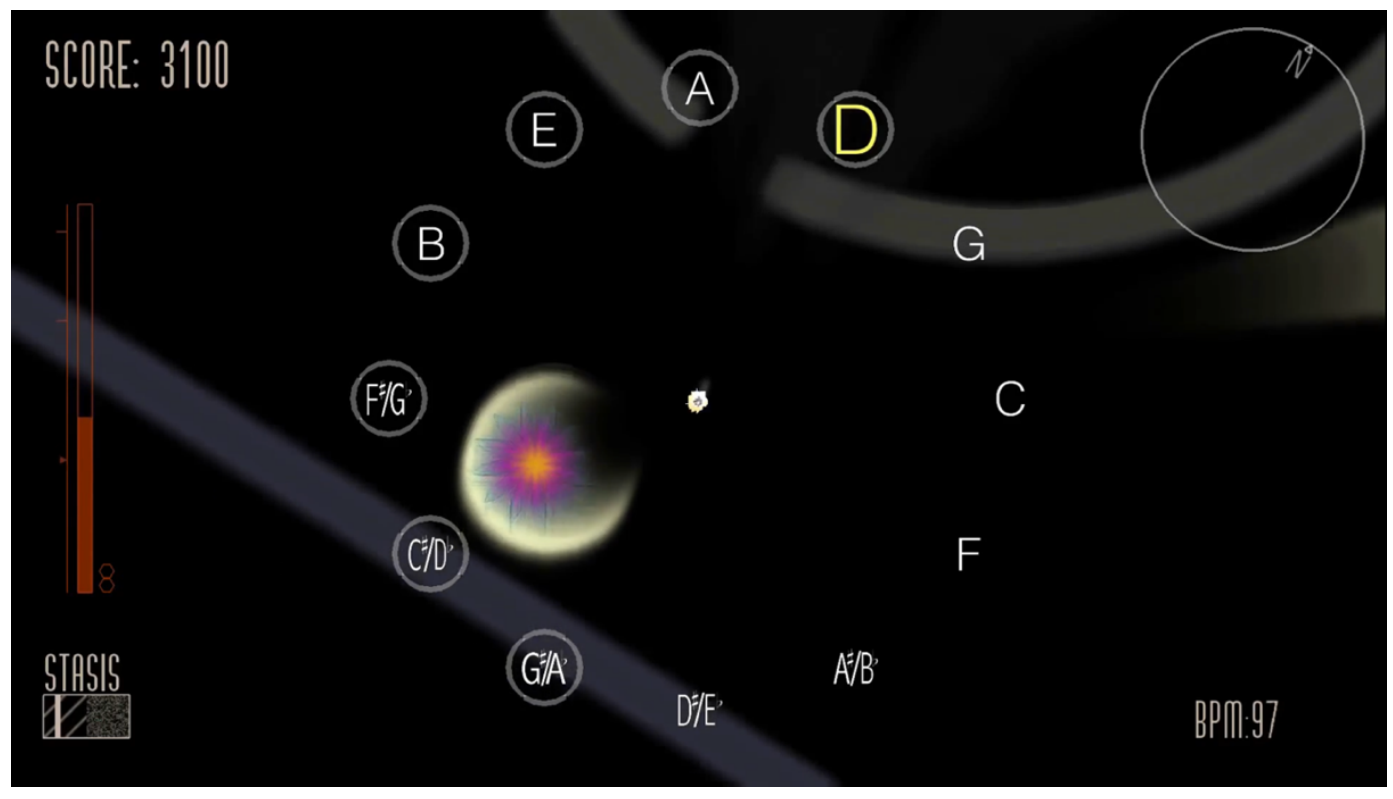

Figure 49. Turowski, Frontier. The outer region

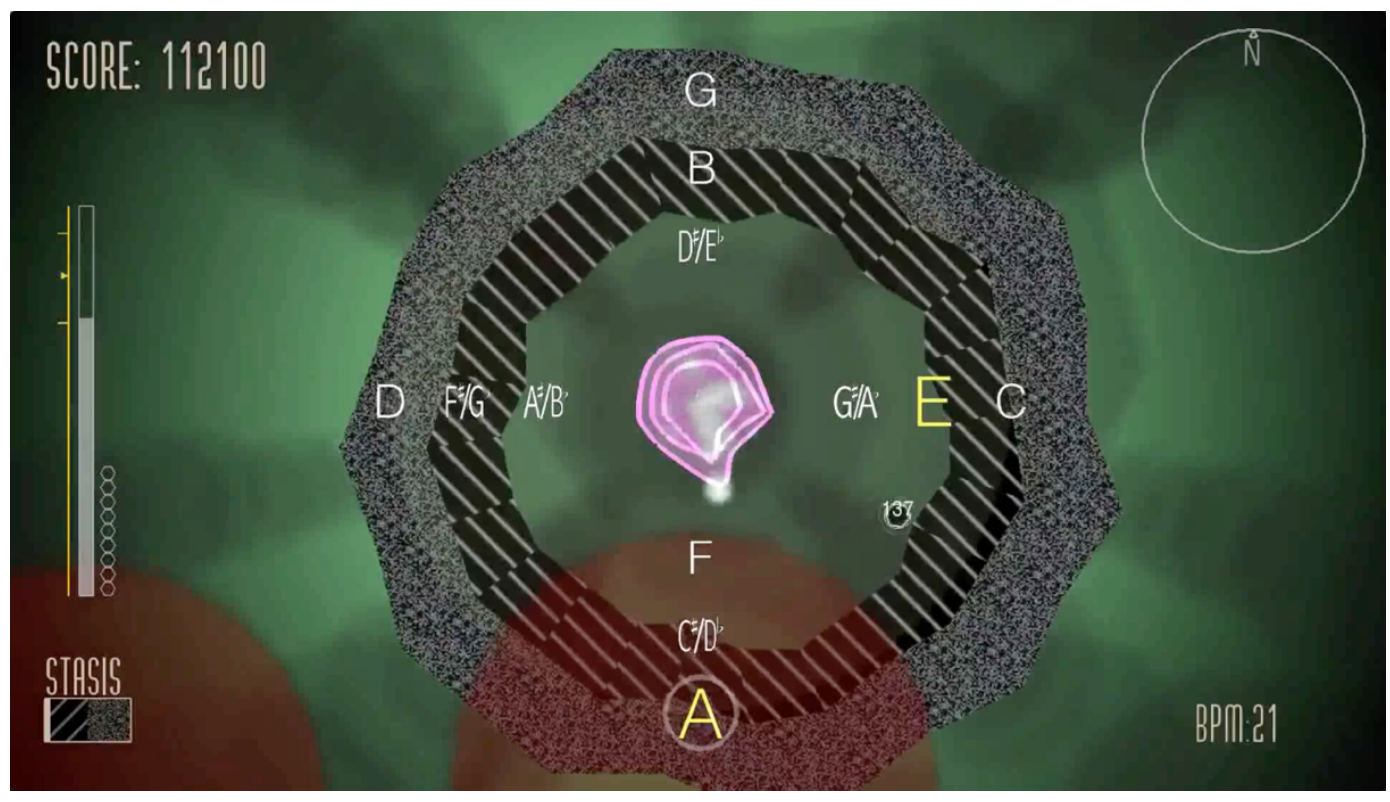

Figure 50. Turowski, Frontier. Stage 3 


\section{Motivations}

Frontier was inspired by the many ideas and examples that have been discussed in previous chapters. It continues a trajectory of development set by Hyperions and borrows many of the same structural features, including a time limit, stage-based progression, and a pointsbased scoring system. It also uses the concept of control sound, enabling a player to directly affect the digital game system without electronic peripherals. However, with Frontier I had several new ideas in mind that can be reduced to three major points:

1) I wanted to make the work more open to different performance contexts by lessening restrictions on who could play. While pitched instruments are still needed, Frontier allows for any number of instrumentalists to perform the piece, which required rethinking many technical aspects of Hyperions - most significantly, the methods of control sound. The desire for more openness also called for a simplification of the technical setup so that the piece could be performed with only a computer and a single microphone.

2) I wanted to enhance the player's experience. This included an expanded world and a stronger sense of agency, which required developing a robust method of score/world traversal. It also meant that I wanted to explore ways that the world could have a bit more of a charming character to increase imaginative immersion.

3) I wanted to allow for more diverse sonic results. While Hyperions was heavily 
contingent on player input, the sonic patterns generated by the score were almost always the same. Repetition helped to establish a sonic identity for the piece, but I wanted to see if more relaxed constraints might yield more interesting and surprising musical results. In Frontier, I also added an option to export sound cues via OpenSoundControl (OSG) to allow users to customize their own sonic textures in other audio environments like Kyma.

\section{Conceptual Framework for Game Design}

Adhering to the notion of experience-driven design, as prescribed by the MDA model, I began the design process by considering which aesthetic goals would be primary. Since I imagined the experience being collaborative and improvisatory, fellowship ("Game as social framework") was of utmost importance. The mechanics would encourage teamwork and the system of control sound would have to accommodate various configurations so that any musician could participate. I also decided that I would emphasize discovery ("Game as uncharted territory") and challenge ("Game as obstacle course"). As such, the artifact would need to regulate access in a controlled manner and include a sufficient level of obstacles to create conflict.

With Frontier, I aimed for a more balance between accuracy and sandbox musical gameplay styles. Clear quantifiable goals would provide a sense of objective successfailure and contribute to challenge-based immersion, while an open possibility space would allow performers to roam freely inside the game world - in any direction and at 
their own collective pace.

\section{Mechanics and Dynamics}

As I have illustrated, the rules of Frontier can be learned by way of affordances and constraints, which are conveyed by particular correspondences between player input and feedback. While referring to textual instructions certainly speeds up the learning process, the score is designed to be didactic such that a player may begin playing with no previous knowledge and deduce appropriate behavior based on the interactions taking place.

\section{Pitch Consonance : Movement}

The primary and most important game mechanic of Frontier is avatar movement in any direction on a two-dimensional plane. Movement toward and collision with artifacts is the only way to advance to the next stage, and movement toward hive walls and NPGs make energy and point gains possible. Additionally, movement is restricted via virtual constraints. For example, it is not able to pass through NPGs or certain boundaries like the outer walls. These pseudo-physical constraints in the game world enforce the musical rules of the piece, which shape the sonic outcome.

Players are able to move by making pitched sounds that fall within the control range. The default control range is C3 to G6, though this range can be reduced in the Settings menu. (See "Developing the Artifact" below.) Pitches are determined via a bank of 
narrow band pass filters instead of a pitch detection algorithm (e.g. the sigmund object in Pd, which was used for Hyperions.) This allows for multiple pitches to be detected and thus a larger ensemble size. ${ }^{196}$ Additionally, the process of playtesting Hyperions revealed that the natural harmonics of the cello sometimes resulted in false fundamental pitch detection. This issue was addressed in Frontier by writing a simple algorithm that determines consonance with each pitch class. When players play pitches that are consonant (unison, octaves, 5th, 4ths) with a particular (fully formed) limb's pitch, that limb generates energy to boost. 3rds and 6ths are also factored into the consonance calculation, but they are weighted slightly less. The consonance algorithm also allows the players more freedom in choosing which notes to play while controlling the avatar.

The movement mechanic also determines more complex harmonic relationships between different players. In the first stage, when there is only one layer to the avatar, players are able to move the avatar in a particular direction by playing pitches that average the desired direction. For example, playing A and E together results in movement vector that is the sum of the two. (Figure 47) The same vector could be created by playing different consonances ( $\mathrm{B}$ and $\mathrm{D}$ or $\mathrm{F} \#$ and $\mathrm{G})$, though the length of the summed vector is lessened as the component vectors approach opposing directions. This consonance-based movement design becomes more complex in subsequent stages since the pitches exist on multiple independently rotating layers. Also, since the angles between pitches increase

196 The sigmund object does allow for multiple pitches to be detected, however this particular functionality proved to less robust and more computationally expensive in practice than the use of a bank of band-pass filters. 
with each level, combinational vectors become more relevant.

\section{Loudness : Energy}

Loudness within the control range controls how much energy the avatar expends and how quickly it moves. Loudness and energy are both represented by the meter on the left side of the screen; loudness is depicted by the arrow on the left side of the meter and available energy reserves are depicted by the lighter inner region of the meter as well as the hexagonal units in the bottom right, which each represent one full meter worth of energy. Additionally, there are three levels to energy expenditure; each successive level increases boost amount and rate of energy depletion. (Figure 51)

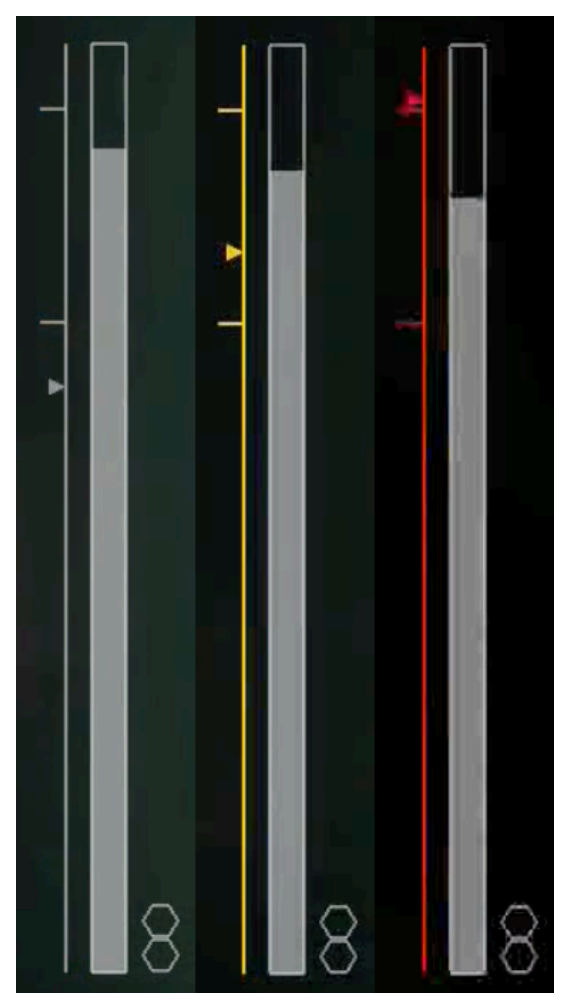

Figure 51. Turowski, Frontier. Three loudness levels 
Beyond the initialization phase (i.e. after the first limb has fully grown), completely exhausting the energy meter (including reserves) will result in avatar destruction. (When the energy meter is close to depletion, the meter blinks red and a sound cue warns players.) When players are silent, energy replenishes; the closer to center of the map the avatar is, the faster the regeneration rate. Additionally, in later stages there are moving red zones that sap avatar energy (even when players are silent). In musical terms, a limited energy reserve serves to regulate the rate of dynamic development. Sustained loudness for too long is penalized with loss of progress, and careful consideration of silence prevents this punishment.

\section{Event Density : NPC Alliances}

A secondary, but important, game mechanic in Frontier is the alliance of NPCs via event density matching (an accuracy-based game objective) to gain energy reserves. Each NPC is labeled with a tempo in BPM (beats per minute) and generates concentric circles at this rate. NPCs that guard the outer gates are larger, more persistent, and have relatively higher BPM values. As such, they are worth three reserve energy units instead of just one. A smoothed measure of the event density (note onsets in BPM) is displayed in the bottom right area of the screen. Additionally, discrete onsets by the ensemble are also represented as concentric circles that radiate outward from the avatar. Matching an NPC's tempo within 10 BPM for a few seconds causes the NPC to become an ally; the smaller the difference in BPM, the faster the NPC is allied. NPCs are only affected if they 
are within range, which is depicted by a translucent golden circle surrounding the avatar. (Figure 47) Agitated NPCs will ram the avatar, potentially changing its current orientation and trajectory (setback punishment).

Devoting time to securing NPG allies allows the players to venture further from the center without fear of dying by way of energy depletion. Musically, this BPM matching mechanic serves to distinguish hives and bases from all other regions of the map. Since the BPM values of guard NPGs are relatively higher than normal NPGs, a more energetic playing style is needed to turn them to allies. Furthermore, BPM values increase with each successive stage, resulting in an overall increase in rhythmic intensity over time.

\section{$\underline{\text { Hives and Spectral Flux }}$}

The third primary game mechanic that informs player interactions is the mapping of spectral flux (a measure of how much the sound's spectrum changes over time) to the ability of the avatar to penetrate hive walls. The meter in the bottom-left corner of the screen shows whether player input is spectrally stable (striped pattern) or in a state of flux (noise-based pattern). A signal with a high degree of flux might be noisy, a quickly changing melodic pattern, or a rhythmic pattern with a high tempo. Each block of a hive wall is textured with a pattern that corresponds to the appropriate amount of spectral flux needed to penetrate it most quickly. For example, a block with a noisy pattern will be destroyed immediately if the flux meter is on the right and the avatar collides with it, while it will take several hits to destroy the block if the flux meter is on the left. Black 
blocks are neutral. Musically, the spectral flux mechanic guides shifts in timbre and activity level, and, since block features are randomly generated, adds an unpredictable element to the musical score. From a game-design perspective, it reinforces accuracybased micro-objectives (rules) by controlling access via constraints, thus implementing a setback punishment for failure.

\section{$\underline{\text { Macro-Objectives }}$}

Perhaps the most intuitive objective of Frontier is to stay alive. In Juul's terminology, dying in Frontier is neither an energy punishment, life punishment (loss of a "life" unit, of which there are none), nor a game termination punishment. Rather, dying is mostly a setback punishment, causing the avatar to be reset to the starting point. All other assets gained (points, energy reserves, stage progression) are maintained. However, dying also results in a point deduction (see below).

Another class of potential objectives relates to time. Players might be motivated to complete all four stages before time runs out. This is achieved by successfully finding all four artifacts (i.e. the beings that warp the avatar to the next stage). They might also try to complete all four stages as fast as possible, perhaps with the goal of trying to beat a previous time or another group's best time.

Players may also choose to regard the points-based scoring system as a game-based objective. The presence of a score meter in the upper left corner and a breakdown of the 
final point tally at the end imply that the scoring system is somewhat important (Figure 52), but players are told explicitly in the work's text-based instructions that they may ignore points if they want. The points breakdown is as follows:

Each block destroyed is worth 100 pts.

Each ally gained is worth 1,000 pts.

Larger guard allies are worth 3,000 pts.

Each artifact found is worth 50,000 pts.

Each life lost subtracts 1000 pts. from the total

A life is lost if the timer runs out

Each second left after the game is worth 100 pts.

If players decide to use the points systems as a goal, then certain strategic considerations come into play. The high value of artifacts makes stage advancement important, and the number of seconds remaining upon completion is factored into the score. However, an optimal points-focused playthrough is not necessarily one in which players rush to finish all four stages as quickly as possible since points are given for cleared blocks and allied NPGs. In the first stage alone, there anywhere from 16 to 96 hive blocks $(1600-9600$ pts.) per hive. There are 6 hives (9600 - 57600 block pts. total), each of which contain 3 NPGs (18,000 pts. total). In later stages, the number of blocks and NPGs generally increase. In retrospect, the scoring system could be further tuned to make a slower and more thorough playthrough potentially more lucrative, thus rewarding different strategic decisions. One simple way to achieve this would be to reduce the value of each remaining second, which would increase the relative value of blocks and NPGs.

The ideal scenario for a truly improvised performance of Frontier is that all of these macroobjectives create an open system in which players may collectively oscillate between 
making aesthetically-pleasing musical decisions and achieving any of multiple game-based objectives. Sandbox-style openness is important to facilitate musical authorship and a greater ontological effect (as Bartee asserts), while the accuracy-based objectives of Frontier limit the possibility space to a composed class of musical goals.

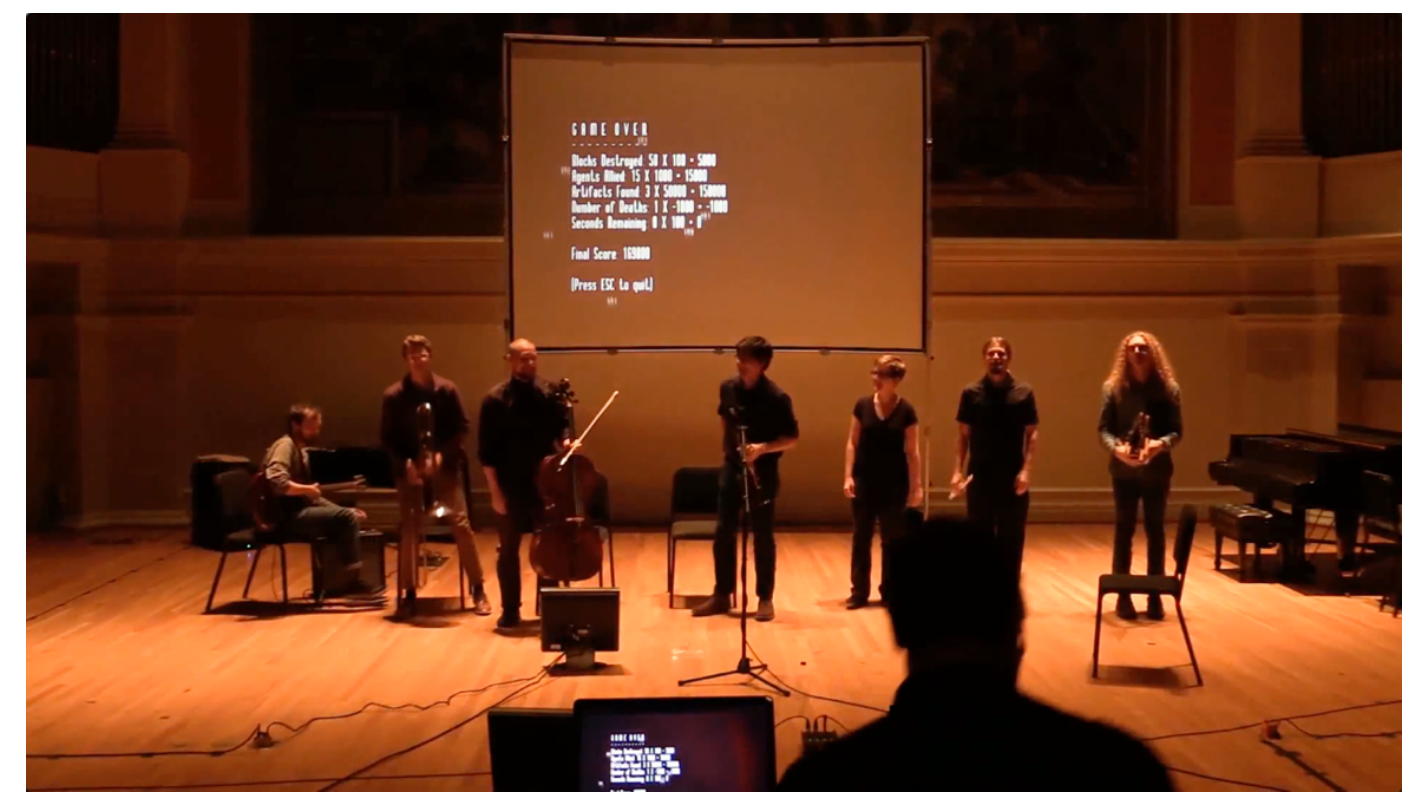

Figure 52. Turowski, Frontier. Final score as the concluding image

\section{Developing the Artifact}

The artifact of Frontier was created in $\mathrm{C}++$ using OpenFrameworks ("an open source C++ toolkit for creative coding" ${ }^{197}$ ). Native libraries include utility functions like OSC (OpenSoundControl) message handling, which was used to allow for external sound cues

\footnotetext{
197 OpenFrameworks web site, http://openframeworks.cc/ [accessed 2016FEB08].
} 
in Kyma. Additionally, many freely available user-created libraries (called "addons") greatly facilitated Frontier's creation. All physics simulations were achieved by way the ofxBox2d addon by Todd Vanderlin ${ }^{198}$, which allowed for the easy insertion of Erin Catto's Box2d physics library ${ }^{199}$. The ofxPd library by Dan Wilcox ${ }^{200}$ allowed for Pd patches to be embedded in the software. This allowed for dynamic sounds and robust real-time analysis via Pd objects such as Brent's timbreID. Since all of these libraries are open source, I was able to customize them to fit the design of Frontier as needed.

The artifact is designed so that several parameters may be customized beforehand. There are many variables involved with control sound, and different configurations of instruments, audio equipment and physical space will produce different analytical results. To account for such variance, a calibration screen is provided which allows users to set parameters such as pitch range and thresholds for loudness, event density, and spectral flux.

\footnotetext{
198 ofxBox2d GitHub repository, https://github.com/vanderlin/ofxBox2d [accessed 2016FEB08].

199 Erin Catto, Box2d web site, http://box2d.org/ [accessed 2016FEB08].

200 ofxPd GitHub repository, https://github.com/danomatika/ofxPd [accessed 2016FEB08].
} 


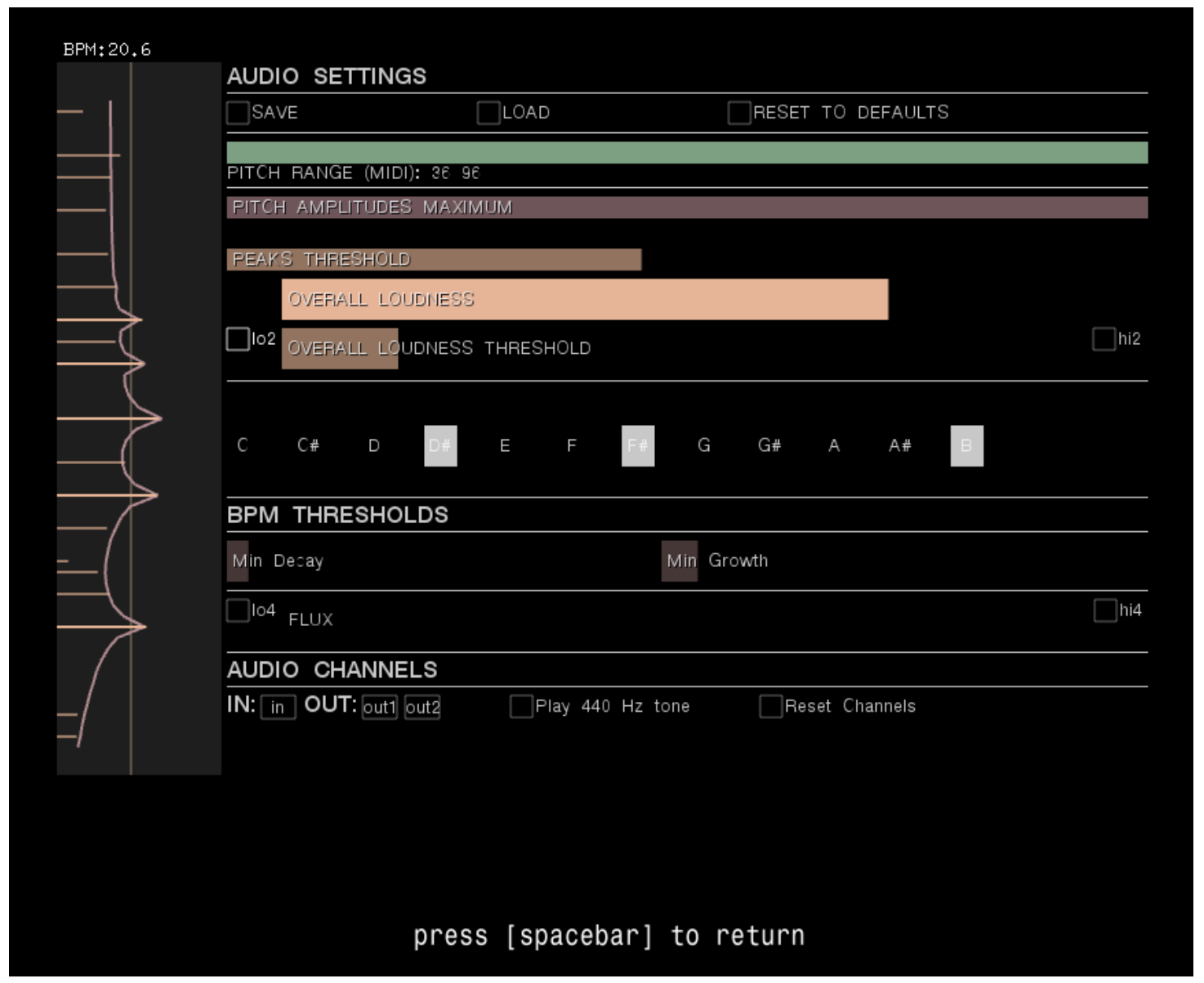

Figure 53. Turowski, Frontier. Calibration screen

User-defined variables are also available on the title screen (Figure 54), which allow for the performance to be more generally customized. These include toggles for a tutorial mode and fullscreen mode as well as number slider for variable game length (3 to 30 minutes). 


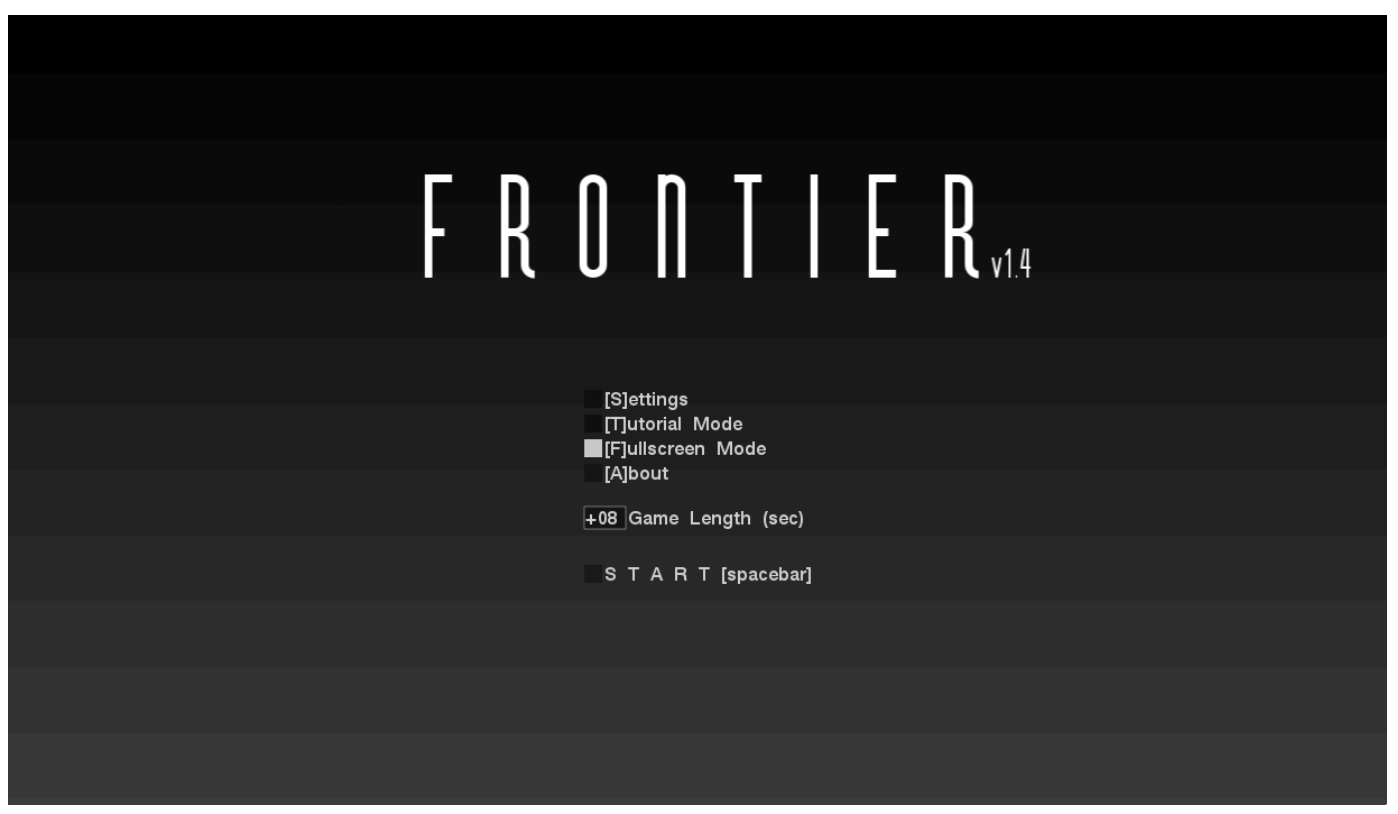

Figure 54. Turowski, Frontier. Title screen

Like Hyperions, Frontier was developed by way of playtesting. Honed over several iterations by different performing groups, including SoundProof and the University of Virginia's New Music Ensemble, it went through many changes and saw much improvement - both as a technical mechanism and an aesthetic trace. (Figure 55) Technically, the process of playtesting with various instruments in different settings was indispensable for tuning the control sound mechanic (and there is still much work to be done). Aesthetically, I was able to shape the scale and configuration of the structure intuitively such that performances approached the musical result ("a class of goals") that I wanted. While I'm pleased with the way that Frontier has evolved over more than a year's worth of development and refinement, there are still a number of revisions to complete before public release. (See "Future Directions" below.) 


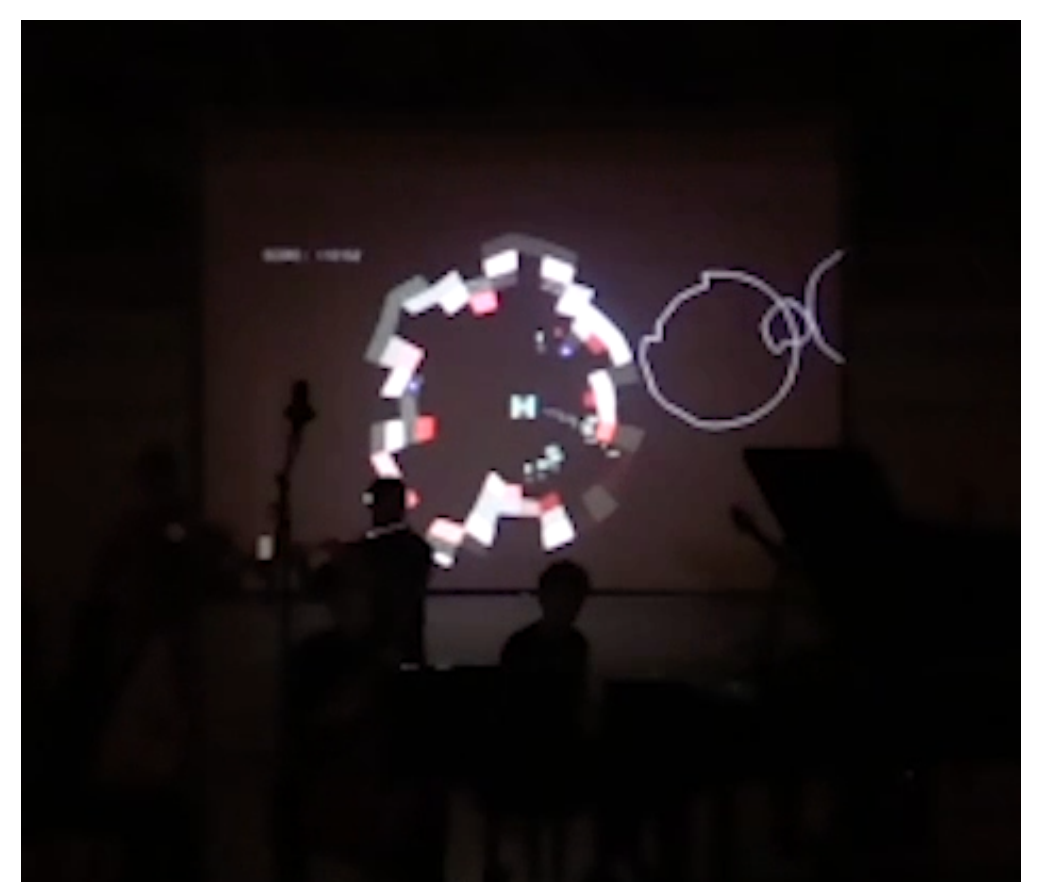

Figure 55. One of the earliest iterations of Frontier

\section{Summary}

Frontier is a digital game piece that affords a particular range of musical outcomes while providing for a significant amount of player agency. On one hand, there is a composed predictable structure that stabilizes the experience of playing and strengthen the players' conceptual model of the piece. Furthermore, elastic anchors - such as the animated limbs and particle clouds of the avatar - serve to establish conceptual blends that enhance understanding and imaginative immersion. On the other hand, Frontier allows for players to significantly incorporate their own experiences and contexts into the unfolding of the performed artifact (like the open works that have inspired it). 
Through the lens of Aarseth's typology, we can understand it as both an ergodic and a cybernetic artifact that regulates the multicursal traversal of scriptons by way of both chance and choice. More specifically, it might be classified in the following manner:

1. Dynamics - intratextonic dynamics (textons fixed, variable scripton content)

2. Determinability - indeterminate (scripton adjacency is variable)

3. Transiency - transient (passing of time has effect)

4. Perspective - personal (users identify as a character)

5. Access - controlled (via affordances)

6. Linking - conditional (controlled access to artifacts, which act as links to stages)

7. User Function - configurative (includes explorative and interpretative)

This ergodic analysis - when, for example, compared with the analysis of Wolff's For 1, 2, or 3 People - reveals Frontier unique qualities. Its complex mechanical structure and highly ergodic design are the result of a flat poietic method - i.e. musical composition based on ecologonomy. Furthermore, the artifact itself is actively involved in poiesis, whether framed as an extension of the composer (I.A.) or as an independent agent (OOO). This method of musical notation accommodates desires for play ("manipulation that indulges curiosity") and fun ("pleasure with surprises"), explores new modes of collaborative authorship, and facilitates ontological exploration and emergent generative gardens shaped by classes of goals.

\section{Future Directions}

After the development and several performances of Frontier, one of the areas I plan to explore further in revisions as well as in future digital game pieces is player immersion. As Ermi and Mäyrä suggest, sensory immersion correlates to "audiovisual quality and 
style," ${ }^{201}$ which may be enhanced by high-resolution graphics, surround sound, and emerging technologies like virtual reality. I am particularly excited about the potential of augmented reality (digital worlds blended with real-world environments) and plan to explore its utility in a musical performance setting. Naturally, its implementation would be informed by existing interface design principles like direct manipulation, kinesonic synchresis, and the elastic anchor to establish strong conceptual models and blends.

I'm also interested in exploring ways to increase imaginative immersion, which could allow players to feel more connected to the game. This might include the use of more representational graphics and sound to connect the virtual to the real, as well as plot and character development. Additionally, larger and more complex game worlds could contribute to a greater sense of wonder and discovery for exploration-centric works like Frontier.

Flow and challenge-based immersion (perhaps the most relevant type of immersion to the unique qualities of digital game pieces) are major considerations for future work. I plan to explore more nuanced systems of challenge, reward, and endogenous value, such as character ability upgrades, hidden areas, and more complex interactions between players and NPCs. With respect to Frontier, I'd like to broaden the flow channel, which includes 1) the development of more intuitive methods for teaching players (and audience members) how to play with minimal rule-based instructions, as well as 2) the use of

201 Ermi and Mäyrä, "Fundamental Components of the Gameplay Experience: Analysing Immersion," 6-7. 
dynamic difficult adjustment to increase challenge for experienced players. More generally, I will continue to refine my current methods of control sound. This will likely include the development of more robust input detection algorithms and systems, the use of convenient peripherals, and more sonic input isolation (as in Hyperions), which would allow for more experimentation with artifact-produced sound since audio feedback would be reduced. For new pieces, the details of the performance system will depend on the ensemble, the desired aesthetic goals, and the technical resources available. In any case, material simplicity remains an important principle. (See "Motivations" above.)

In future work, I also aim to increase poietic efficiency. While I have worked with game engines before (e.g. Unity), their proprietary nature and often expensive licenses have been somewhat discouraging. However, game engines have increasingly become more powerful, more open, and more affordable, making them more suitable for the creation of digital game pieces. Additionally, audio middleware (e.g. Wwise) seems to be following the same trend. In general, specialized game authoring software could help to streamline the creation and distribution (i.e. more cross-platform content) process.

Lastly, the potential for future scholarship on the present topic is vast. Many existing digital games demonstrate excellent design and execution in various ways (e.g. immersion), and a better understanding of such games would strengthen the conceptual framework and poietic methodology for digital game pieces. Also, a more focused study of improvisation and open works might provide additional insights into the esthesic 
process for players of interactive performed artifacts. In my future scholarly research, I plan to conduct more detailed case studies and to delve deeper into the many interrelated concepts of this rich and evolving field.

In conclusion, this project seeks a harmonious balance of digital gaming and musicking. Based on my research and practical experiences, I am confident that continued engagement with this particular interdisciplinary intersection is beneficial to larger artistic discourses on composition and performance. Though it is a natural extension of other preexisting formats (open works, game pieces, etc.), the digital game piece affords novel poietic experiences (for composer and performer alike) that will inevitably inform and expand other creative practices, including those from which it derives. 


\section{Bibliography}

Aarseth, Espen J. "Computer Game Studies, Year One." The International Fournal of Computer Game Research, Vol. 1, Issue 1, (July 2001).

- Cybertext: Perspectives on Ergodic Literature. Baltimore, London: Johns Hopkins UP, 1997.

Agawu, Kofi. Music as Discourse: Semiotic Adventures in Romantic Music. New York: Oxford UP, 2009.

Arsenault, Dominic. "Video Game Genre, Evolution and Innovation." Eludamos. Fournal for Computer Game Culture, Vol. 3, No. 2 (2009).

Bahn, Curtis Robert. "Composition, improvisation and meta-composition." Ph.D. diss., Princeton University, 1998. In ProQuest Dissertations and Theses, http://search.proquest.com/docview/304373240?accountid=14678 [accessed November 10, 2014].

Bailey, Derek. Improvisation: Its Nature and Practice in Music. New York: Da Capo, 1992.

Barrett, G. Douglas and Michael Winter. "LiveScore: Real-Time Notation in the Music of Harris Wulfson." Contemporary Music Review, Vol. 29, No. 1 (February 2010).

Bartee, Jordan. "Ontological Toys.” PhD diss., Brown University, 2014.

Barthes, Roland. "The Death of the Author." UbuWeb. http://www.ubu.com/aspen/aspen5and6/threeEssays.html [accessed 2016FEB01].

Bateson, Gregory. Mind and Nature: A Necessary Unity. E.P. Dutton: New York, 1979.

—. Steps to an Ecology of Mind. Chicago, London: Chicago UP, 2000.

Bergson, Henri. Matter and Memory. Trans. Nancy M. Paul and W. Scott Palmer. New York, 1911 [orig. French 1896].

Bergun, Keith. "The Four Interactive Forms." http://keithburgun.net/interactiveforms/ [accessed 2016FEB01].

Bogost, Ian. Alien Phenomenology, or What It's Like to Be a Thing. Digital edition. Minneapolis: Minnesota UP, 2012. 
Bogost, Ian. "Gamification is Bullshit." The Atlantic web site. http://www.theatlantic.com/technology/archive/2011/08/gamification-isbullshit/243338/. Accessed on 11OCT2014.

Braxton, Anthony. Tri-axium writings: Vol. 1. Oakland: Frog Peak Music, 1985.

Brent, William. "Research." Web page. http://williambrent.conflations.com/pages/research.html [accessed 2016FEB01].

Bryant, Levi. The Democracy of Objects. Ann Arbor: Open Humanities Press, 2011.

_ . "Flat Ontology." Larval Subjects. https://larvalsubjects.wordpress.com/2010/02/24/flat-ontology-2/, [accessed 2016JAN30].

Burtner, Matthew. "Metasax." http://matthewburtner.com/metasax/ [accessed 2016FEB08].

Cage, John. "Composition as Process: Indeterminacy." Audio Culture: Readings in Modern Music. Eds. Christopher Cox and Daniel Warner. New York, London: Continuum, 2004, pp. 176-88.

Cage, John and Lukas Foss. Concerto for Prepared Piano \& Orchestra/Baroque Variations. Liner notes. Nonesuch H-71202, Vinyl LP, 1968.

Caillois, Roger. Man, Play and Games. Trans. Meyer Barash. Urbana, Chicago: Illinois UP, 2001.

Cardew, Cornelius. Treatise Handbook. London: Peters Edition, 1971.

Carl, R. "Christian Wolff: On Tunes, Politics, and Mystery." Contemporary Music Reviewe, vol. 20, no. 4 (2001).

Chadabe, Joel. Electric Sound: The Past and Promise of Electronic Music. New Jersey: PrenticeHall, 1997.

Chen, Jenova. "Flow in Games." master's thesis, University of Southern California, 2006. http://www.jenovachen.com/flowingames/designfig.htm [accessed 2016FEB06].

Chion, Michel. Audio-Vision: Sound on Screen. Ed. and trans. Claudio Gorbman. New York: Columbia UP, 1990. 
Chow, Kenny K. N. and D. Fox Harrell. "Material-Based Imagination: Embodied Cognition in Animated Images." In Proceedings of the Digital Arts and Culture Conference, UG Irvine, CA, USA, 2009.

Clark, Herbert H. Using Language. Cambridge: Cambridge UP, 1996.

Collins, Karen. Game Sound: An Introduction to the History, Theory, and Practice of Video Game Music and Sound Design. Cambridge: MIT Press, 2008.

—. Playing with Sound. Cambridge: MIT Press, 2013.

Cook, Nicholas. "Between Process and Product: Music and/as Performance." Music Theory Online, Vol. 7, No. 2 (April 2001).

Cope, David. New Directions in Music, 4t ${ }^{\text {th }}$ ed. Dubuque: Brown, 1984.

Crawford, Chris. The Art of Computer Game Design. Washington State University, 1997. Digital edition.

Csikszentmihalyi, Mihaly. Flow and the Foundations of Positive Psychology: The Collected Works of Mihaly Csikszentmihalyi. Springer: New York, 2014.

DeLanda, Manuel. Intensive Science and Virtual Philosophy. London: Continuum, 2002.

DeLio, Thomas. Circumscribing the Open Universe, University Press of America, 1984.

Derrida, Jacques. Memoires for Paul de Man, revised edition. Columbia UP, 1989.

Deterding, Sebastian, Dan Dixon, Rilla Khaled, and Lennart Nacke. "From Game Design Elements to Gamefulness: Defining 'Gamification'.” In Proceedings of the 15th International Academic MindTrek Conference: Envisioning Future Media Environments (MindTrek '11). ACM, New York, NY, USA, 2011, pp. 9-15.

DeVeaux, Scott and Gary Giddins. Fazz. New York, London: Norton, 2009.

Doob, Penelope Reed. The Idea of the Labyrinth from Classical Antiquity through the Middle Ages. Ithaca: Cornell UP, 1990.

Duffie, Bruce. "Earle Brown Interview with Bruce Duffie." Radio interview transcription on web site. http://www.bruceduffie.com/brown.html [accessed on 5DEC2014].

Eco, Umberto. The Open Work. Cambridge: Harvard UP, 1989.

—.A Theory of Semiotics. Bloomington: Indiana UP, 1976. 
Eno, Brian. "Composers as Gardeners." Edge. http://edge.org/conversation/composersas-gardeners [accessed on 5SEPT2015].

. "Generating and Organizing Variety in the Arts." Audio Culture: Readings in Modern Music. Eds. Christopher Cox and Daniel Warner. New York, London: Continuum, 2004, pp. 226-33.

Ermi, Laura and Frans Mäyrä. "Fundamental Components of the Gameplay Experience: Analysing Immersion." Changing Viewes: Worlds in Play. Selected Papers of the 2005 Digital Games Research Association's Second International Conference, Eds. Suzanne de Castell and Jennifer Jenson.

Fauconnier, Gilles and Mark Turner. The Way We Think. New York: Basic Books, 2002.

Feldman, Morton. Essays. Beginner Press, 1985.

Foucault, Michel. "What is an Author?" The Foucault Reader. Ed. Paul Rabinow. Michigan: Pantheon, 1984.

Frederic, Gerald. "The African Aesthetic in World Creativity: Anthony Braxton's Philosophy of Vibrational Affinity Dynamics." Journal of Black Studies, Vol. 38, No. 2 (November 2007), pp. 130-141.

Fuster, Joaquin. Cortex and Mind: Unifying Cognition. New York: Oxford UP, 2003.

—_. "Distributed Memory and the Perception-Action Cycle." Recorded lecture from Conference on Brain Network Dynamics held at the University of California at Berkeley on January 26-27, 2007.

https://archive.org/details/Brain_Network_Dynamics_2007-13-Joaquin_Fuster [accessed on Sept. 9, 2015].

Galloway, Alexander. Gaming: Essays on Algorithmic Culture. Minnesota UP, 2006.

Gingold, Chaim. "Miniature Gardens \& Magic Crayons: Games, Spaces, \& Worlds." master's thesis, Georgia Institute of Technology, 2003. http://levitylab.com/cog/writing/thesis/ [accessed 2016JAN30].

Goehr, Lydia. The Imaginary Museum of Musical Works: An Essay in the Philosophy of Music. Oxford: Clarendon Press, 1992.

Griffiths, Paul. Modern Music and After: Directions Since 1945. New York: Oxford UP, 1995.

Halbwachs, Maurice. The Collective Memory. Trans. Francis J. Ditter, Jr. and Vida Yazdi Ditter. New York: Harper \& Row, 1980 [orig. French 1950]). 
Hayles, N. Katherine. How We Became Posthuman: Virtual Bodies in Cybernetics, Literature, and Informatics. Chicago UP, 1999.

Herber, Norbert. "The composition--instrument: emergence, improvisation and interaction in games and new media." From Pac-Man to Pop Music: Interactive Audio in Games and New Media. Ed. Karen Collins. Farnham, Surrey, GBR: Ashgate Publishing Group, 2008. Chapter 7, pp. 103-23.

Holtzman, Stephen. Digital Mantras. Cambridge: MIT Press, 1996.

Hope, Cat and Lindsay Vickery. "Screen Scores: New Media Music Manuscripts." Paper presented at the annual meeting of the International Computer Music Conference, University of Huddersfield, UK, 31 July - 4 August 2011.

Huizinga, Johan. Homo Ludens: A study of the Play-Element in Culture. London: Routledge \& Kegan Paul, 1949.

Hunicke, Robin, Marc LeBlanc, Robert Zubek. "MDA: A Formal Approach to Game Design and Game Research." Proceedings of the Challenges in Games AI Workshop, Nineteenth National Conference of Artificial Intelligence, 2004.

Ingarden, Roman. The Work of Music and the Problem of Its Identity. Trans. Adam Czerniawski. Ed. Jean G. Harrell. Berkeley, Los Angeles: UC Press. 1986 [orig. Polish 1966].

Inwood, M. J. A Hegel Dictionary. Wiley-Blackwell, 1992.

Ives, Charles. "Postface to 114 Songs." Essays before a Sonata, and Other Writings. Ed. Howard Boatwright. New York: Norton, 1961, pp. 120-31.

Iwai, Toshio. Iwai Toshio no shigoto to shuhen. Tokyo: Rikuyosha, 2000.

Jackowski, Dariusz et al. "Computer Game Piece: Exploring Video Games as Means for Controlled Improvisation." Proceedings of International Computer Music Conference, Athens, Greece, 2014.

Juul, Jesper. "Fear of Failing? The Many Meanings of Difficulty in Video Games." Eds. Mark J. P. Wolf \& Bernard Perron. The Video Game Theory Reader 2. New York: Routledge 2009. pp. 237-252.

Kanaga, David. "Intro to Ludic Ecologonomy (Pt. 1)." Wombflash Forest http://wombflashforest.blogspot.com/2015/04/intro-to-ludic-ecologonomy-pt-

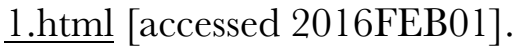


Kayali, Fares. "Playing Music: Design, Theory, and Practice of Music-based Games." PhD diss., Vienna University of Technology, 2008.

Kierkegaard, Søren. Either/Or. Trans. D. F. Swenson and L. M. Swenson. Princeton: Princeton UP, 1944.

Kim-Boyle, David. "Real-time Score Generation for Extensible Open Forms." Contemporary Music Review, vol. 29, no. 1 (2010).

Lewis, George E. "Improvised Music after 1950: Afrological and Eurological Perspectives." Black Music Research Fournal, Vol. 16, No. 1, (Spring, 1996). pp. 91122.

McCorduck, Pamela. Aaron's Code, Meta-art, Artificial Intelligence, and the work of Harold Cohen. New York: W.H. Freeman and Company, 1991.

McGonigal, Jane. Reality is Broken: Why Games Make Us Better and How They Can Change the World. New York: Penguin, 2011. Digital edition.

Miller, Kiri. Playing Along: Digital Games, YouTube, and Virtual Performance. New York: Oxford UP, 2012. Digital edition.

Morgan, Robert P. Twentieth-Century Music: A History of Musical Style in Modern Europe and America. New York, London: Norton, 1991.

Moseley, Roger. "Playing Games with Music (and Vice Versa): Ludomusicological Perspectives on Guitar Hero and Rock Band." Taking It to the Bridge. Eds. Nicholas Cook and Richard Pettengill. Ann Arbor: Michigan UP, 2013. Digital edition.

Moseley, Roger and Aya Saiki. "Nintendo's Art of Musical Play." Music in Video Games: Studying Play. Eds. K.J. Donnelly, William Gibbons, and Neil Lerner. New York: Routledge, 2014.

Nattiez, Jean-Jacques. Music and Discourse: Toward a Semiology of Music. Princeton: Princeton UP, 1990.

Norman, Don. The Design of Everyday Things, Revised and Expanded Edition. Perseus, 2013.

Phillips, Winifred. A Composer's Guide to Game Music. Cambridge: MIT Press, 2014.

Pichlmair, Martin and Fares Kayali. "Levels of Sound: On the Principles of Interactivity in Music Video Games.” Situated Play, Proceedings of DiGRA Conference, 2007. 
Reale, Steven Beverburg. "Transcribing Musical Worlds; or, Is L.A. Noire a Music Game?" Music in Video Games: Studying Play. Eds. K.J. Donnelly, William Gibbons, and Neil Lerner. New York: Routledge, 2014.

Russolo, Luigi. The Art of Noise (futurist manifesto). Trans. Robert Filliou. (Great Bear Pamphlet, Something Else Press, 1967). UbuWeb. http://www.ubu.com/historical/gb/russolo_noise.pdf [accessed 29NOV2014].

Salen, Katie and Eric Zimmerman. Rules of Play: Game Design Fundamentals. Cambridge: MIT Press, 2004.

Schell, Jesse. Art of Game Design. Amsterdam; Boston: Elsevier/Morgan Kaufmann, 2008.

Shneiderman, Ben and Catherine Plaisant. Designing the User Interface. $4^{\text {th }}$ ed. Boston: Pearson/Addison Wesley, 2005.

Small, Christopher. Musicking. Hanover: Wesleyan UP, 1998.

Smith, Ryan Ross. "An Atomic Approach to Animated Music Notation.” Tenor 2015, Proceedings of the the International Conference on Technologies for Music Notation $\mathcal{E}^{2}$ Representation, Universite Paris Sorbonne/IRCAM, Paris, 2015.

Stone, Kurt. Music Notation in the Twentieth Century: A Practical Guidebook. New York, London: W. W. Norton, 1980.

Suits, Bernard. Grasshopper: Games, Life, and Utopia. Boston: David R. Godine, 1990.

Taylor, Diana. The Archive and the Repertoire. Durham, London: Duke UP, 2003.

Trenkamp, Anne. "The Concept of 'Alea' in Boulez's 'Constellation--Miroir'." Music \& Letters, Vol. 57, No. 1 (Jan., 1976), pp. 1-10.

Trippett, David. “Composing Time: Zeno's Arrow, Hindemith's Erinnerung, and Satie's Instantanéisme." Fournal of Musicology, vol. 24 (2007).

van Dongen, Joost. Cello Fortress web site, http://www.cellofortress.com/ [accessed on Nov. 10, 2014].

Wolff, Christian. For 1, 2, or 3 People. C. F. Peters, 1964.

—. "Open to Whom and to What." Interface vol. 16 (1987), pp. 133--144.

Wulfson, Harris. "LiveScore." http://harris.wulfson.com/?page_id=724 [accessed on Sept 7, 2015]. 
Xenakis, Iannis. Formalized Music: Thought and Mathematics in Composition. Stuyvesant: Pendragon, 1992.

Zorn, John. "The Game Pieces.” Audio Culture: Readings in Modern Music. Eds. Christopher Cox and Daniel Warner. New York, London: Continuum, 2004, pp. 196-200. 